\title{
Algorithmic Issues in some Disjoint Clustering Problems in Combinatorial Circuits
}

\author{
Zola Nailah Donovan \\ West Virginia University, zdonovan@mix.wvu.edu
}

Follow this and additional works at: https://researchrepository.wvu.edu/etd

Part of the Theory and Algorithms Commons

\section{Recommended Citation}

Donovan, Zola Nailah, "Algorithmic Issues in some Disjoint Clustering Problems in Combinatorial Circuits" (2018). Graduate Theses, Dissertations, and Problem Reports. 3721.

https://researchrepository.wvu.edu/etd/3721

This Dissertation is protected by copyright and/or related rights. It has been brought to you by the The Research Repository @ WVU with permission from the rights-holder(s). You are free to use this Dissertation in any way that is permitted by the copyright and related rights legislation that applies to your use. For other uses you must obtain permission from the rights-holder(s) directly, unless additional rights are indicated by a Creative Commons license in the record and/ or on the work itself. This Dissertation has been accepted for inclusion in WVU Graduate Theses, Dissertations, and Problem Reports collection by an authorized administrator of The Research Repository @ WVU.

For more information, please contact researchrepository@mail.wvu.edu. 


\title{
Algorithmic Issues in some Disjoint Clustering Problems in Combinatorial Circuits
}

\author{
Zola Nailah Donovan \\ Dissertation submitted \\ to the College of Engineering and Mineral Resources \\ at West Virginia University \\ in partial fulfillment of the requirements for the degree of \\ Doctor of Philosophy in \\ Computer Science \\ K. Subramani, Ph.D., Chair \\ David W. Graham, Ph.D. \\ Vahan V. Mkrtchyan, Ph.D. \\ Daryl S. Reynolds, Ph.D. \\ Frances L. VanScoy, Ph.D. \\ Lane Department of Computer Science and Electrical Engineering \\ Morgantown, West Virginia \\ 2018
}

Keywords: Disjoint clustering, computational complexity, algorithms, combinatorial optimization

Copyright 2018 Zola Nailah Donovan 


\begin{abstract}
Algorithmic Issues in some Disjoint Clustering Problems in Combinatorial Circuits

Zola Nailah Donovan

As the modern integrated circuit continues to grow in complexity, the design of very large-scale integrated (VLSI) circuits involves massive teams employing state-of-theart computer-aided design (CAD) tools. An old, yet significant CAD problem for VLSI circuits is physical design automation. In this problem, one needs to compute the best physical layout of millions to billions of circuit components on a tiny silicon surface. The process of mapping an electronic design to a chip involves several physical design stages, one of which is clustering. Even for combinatorial circuits, there exist several models for the clustering problem. In particular, we consider the problem of disjoint clustering in combinatorial circuits for delay minimization $(\mathrm{CN})$. The problem of clustering with replication for delay minimization has been well-studied and known to be solvable in polynomial time. However, replication can become expensive when it is unbounded.

Consequently, $\mathrm{CN}$ is a problem worth investigating. In this dissertation, we establish the computational complexities of several variants of $\mathrm{CN}$. We also present approximation and exact exponential algorithms for some variants of $\mathrm{CN}$. In some cases, we even obtain an approximation factor of strictly less than two. Furthermore, our exact exponential algorithms beat brute force.
\end{abstract}


TO MY MOTHER, L. EliZABETH BRYANT-RIVERA 


\section{Acknowledgements}

First and foremost, I would like to give special thanks to my advisor, Dr. K. Subramani, for introducing me to this research topic. He has encouraged me to do rigorous analyses, helped me to overcome challenges and obstacles that I encountered along the way as a Ph.D. student, and overall, inspired me to be a better researcher. I'm tremendously grateful to him for being both accessible and responsive during this entire process. The ease with which I could discuss research with him (via phone, email, or in person) was most meaningful. It seems that there is never a time that he is not thinking about a research problem. I've grown to even think of him as a friend, and I look forward to continued collaboration.

To my committee members: I thank Dr. Vahan Mkrtchyan, a person who was there from the beginning of my research journey, for his kindness, helpfulness, and willingness to always give thoughtful feedback. He has been invaluable to my professional growth, and I am grateful to have him serve on my committee. I offer sincere thanks to Dr. Frances L. VanScoy, Dr. David W. Graham, and Dr. Daryl S. Reynolds, who serve as experts and whose questions I have revisited and considered as I worked through this process.

As a participant of the Summer Faculty Fellowship Program at the Air Force Research Laboratory (accompanying Dr. Subramani for two summers and remaining this fall), I was able to work with a great team of lab mentors Steven Drager and Dr. Matthew Anderson. Many thanks to them for all of their support and for being instrumental in opening doors to new and exciting opportunities at the lab. I also met another great lab mentor, Dr. Timothy Kroecker. His support and encouragement made it possible for me to continue my research. For that, I am incredibly grateful. I thank Steven Farr for bringing me to the lab for employment opportunities earlier this year. Without his efforts and his belief that I am a good fit at the lab, I would not soon call it my new work home with a new work family. Additionally, I extend thanks to Dr. Noor Ahmed for his tremendous support at the lab, Leah Chance for opening her home to me and for our end-of-day chats, Dr. Alvaro Velasquez for our relatable research chats, and Captain Mitch Young for rolling out the welcome mat with all of his kind and positive energy. I sense good times ahead.

I would also like to acknowledge some special folks whom I met during my time at West Virginia University: Dr. Elaine Eschen helped me survive the harrowing CCDM examination process and gave me keen insights into the nuts and bolts of moving suc- 
cessfully towards Ph.D. candidacy. Dr. W. Constinia Charbonnette always believed in and supported me from the day we first met. Life at WVU would not have been the same without knowing I could always count on her. Dr. David Miller, Dr. Jessica Deshler and Dr. Vicki Sealey inspired me to be a better educator. Since working on my master's in the math department, Dr. Harvey Diamond encouraged me to followthrough on my academic goals. I give many thanks to Dr. Charbonnette, Dr. Miller, Dr. Deshler, Dr. Sealey, and Dr. Diamond, for helping to pave the way for me to receive the WVU STEM Mountains of Excellence Fellowship, as well as other funding. Focusing on research and continuing my studies would otherwise have been much more challenging. Marcela Mera Trujillo is a good friend and was the most reliable and consistent study-buddy. Dr. Amy Hunter had numerous study sessions at her home, extended many invites to events and activities that made WVU feel like my home away from home-she is indeed a true friend. They all helped to make this journey memorable.

Last but not least, the love of my family kept me going strong. I especially give thanks to my mother for our weekly talks and her many words of encouragement. She has always given me the best advice and has consistently urged me to keep my eyes steadfastly on the prize. Because of her, I never gave up. I thank Felix for being a very supportive stepdad. Also, knowing that he was taking good care of my mom, allowed me to focus on my studies and research. I thank my sister Aiyana and best friend since Day 1, for her unwavering support. She is one of the most generous and loving people I know. I also appreciate the rest of my extended family for their continued love and support. 


\section{Credits}

The work in this dissertation is the result of collaborative efforts which lead to coauthored papers. These papers are either currently in print [DMS15], accepted pending revisions [DGMS18], or very recently submitted to a high-quality conference with our journal version ready. Here, I give credit to my co-authors, including Dr. K. Subramani (my research advisor), Dr. Vahan Mkrtchyan, and Dr. Gregory Gutin.

This research was supported in part by the Air Force Research Laboratory Information Directorate, through the Air Force Office of Scientific Research Summer Faculty Fellowship Program and the Information Institute, contract numbers FA8750-163-6003 and FA9550-15-F-0001. 


\section{Contents}

Dedication iii

Acknowledgements iv

Credits vi vi v v v

\begin{tabular}{|l|l}
\hline List of Figures & ix
\end{tabular}

List of Tables $\quad$ xi

$\begin{array}{lll}1 & \text { Introduction } & 1\end{array}$

$1.1 \quad$ Clustering when replication is allowed $\ldots \ldots \ldots . . \ldots 4$

1.1 .1 Formulation of $\mathrm{CA} \ldots \ldots \ldots 6$

1.1 .2 An example of CA . . . . . . . . . . . . . . 7

1.2 Motivation and related work $\ldots \ldots \ldots$. . . . . . . . . . 9



2.1 Graph preliminaries . . . . . . . . . . . . . . . . 15

2.2 Formulation of $\mathrm{CN}$ using combinatorial circuits . . . . . . . . . . . . . . 16

2.3 Graph-theoretic formulation of $\mathrm{CN}$. . . . . . . . . . . . . . . 18

$\begin{array}{lll}3 & \text { Computational Complexity } & \mathbf{2 0}\end{array}$

3.1 Computational complexities of several variants of $\mathrm{CN}$. . . . . . . . 20

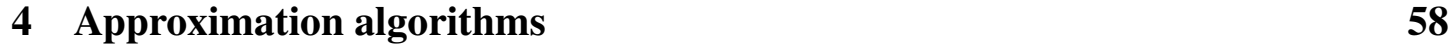

$4.1 \quad$ An integer program for $\mathrm{CN}\langle W, M, \Delta\rangle \ldots \ldots \ldots$. . . . . . . . . 58

$4.2 \quad$ An LP-rounding algorithm for $\mathrm{CN}\langle N, 2, \Delta\rangle \ldots \ldots$. . . . . . . . . . . . . 63

$4.3 \quad$ A 3-approximation algorithm for $\mathrm{CN}\langle N, 2, \Delta\rangle \ldots \ldots$. . . . . . . . . 65

4.4 An improved 2-approximation algorithm for $\mathrm{CN}\langle N, 2, \Delta\rangle \ldots$. . . . . . 68

4.5 A simple $\left(2+\frac{2}{l-1}\right)$-approximation algorithm $\ldots \ldots \ldots . . \ldots 72$

4.6 A parameterized approximation algorithm for $\mathrm{CN}\langle W, M, \Delta\rangle$. . . . . . . 74

\begin{tabular}{|lll}
5 & Exact Exponential Algorithms & $\mathbf{7 7}$
\end{tabular}

$5.1 \quad$ Exact exponential algorithms for $\mathrm{CN}\langle W, M, \Delta\rangle \ldots \ldots$. . . . . . . . 77

$5.2 \quad$ Exact exponential algorithms for $\mathrm{CN}\langle N, 2, \Delta\rangle \ldots \ldots$. . . . . . . 78 
$5.3 \quad$ An exact exponential algorithm for $\mathrm{CN}\langle W, M, 3\rangle \ldots \ldots$. . . . . . . . 80

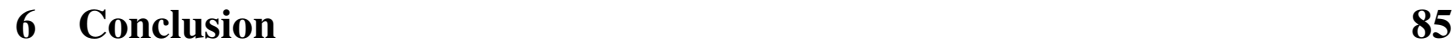

6.1 Summary of contributions . . . . . . . . . . . . . . . 85

6.2 Future work $\ldots \ldots \ldots \ldots$. . . . . . . . . . . . . 87

$6.2 .1 \quad$ Implementation and experimentation . . . . . . . . . . . 88

6.2 .2 Unique Games Conjecture . . . . . . . . . . . . . . . 88

\begin{tabular}{lr}
\hline Bibliography & 91
\end{tabular} 


\section{List of Figures}

$1.1 \quad$ Simple example of clusterings of a directed path. . . . . . . . . . . 2

1.2 An instance of the clustering problem when replication of vertices is

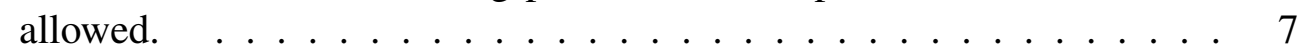

1.3 The $\mathrm{I} / \mathrm{O}$ model $[\mathrm{AB} 10] . \ldots \ldots \ldots$

2.1 A DAG representing a combinatorial network with two sources and two

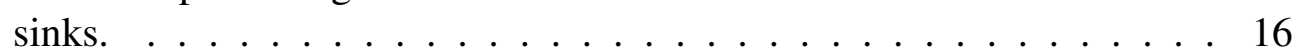

2.2 An example of a DAG and clustering of the DAG. . . . . . . . . . . 19

2.3 Some cases of the delay minimization problem that we investigate in this dissertation. . . . . . . . . . . . . . . . . . 19

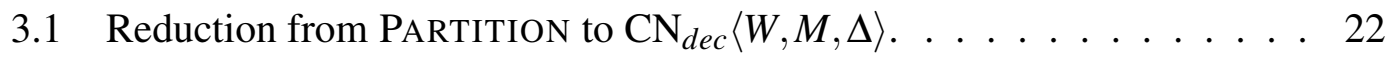

3.2 Reduction from PARTITION to $\mathrm{CN}_{d e c}\langle W, M, 3\rangle . \ldots \ldots . . \ldots . . .25$

3.3 Gadgets used to represent variables and clauses. . . . . . . . . . . . . . 29

3.4 Simple example . . . . . . . . . . . . . . . . . . . . 30

3.5 Reduction from the 3SAT problem to $\mathrm{CN}_{d e c}\langle[3], 3,3\rangle$. The edges connecting variable gadgets to clause gadgets belong in the area with the shaded cloud. . . . . . . . . . . . . . . 31

$3.6 \quad$ Gadgets used to represent variables and clauses. . . . . . . . . . . . . 34

3.7 Reduction from 3-BP 1-IN-3SAT to $\mathrm{CN}_{d e c}\langle[2], 2,4\rangle$. The edges connecting variable gadgets to clause gadgets belong in the area with the shaded cloud. . . . . . . . . . . . . . . . . 34

3.8 Gadgets used to represent variables and clauses. . . . . . . . . . . . . . 38

3.9 Reduction from $1-\mathrm{IN}-3 \mathrm{SAT}$ to $\mathrm{CN}_{\operatorname{dec}}\langle[2], 2,3\rangle$. The edges connecting variable gadgets to clause gadgets belong in the shaded area. . . . . . . 38

3.10 Gadgets used to represent variables and clauses. . . . . . . . . . . . . . . . . 42

3.11 Gadgets used to represent variables and clauses. . . . . . . . . . . . . 44

3.12 Gadgets used to represent variables and clauses. . . . . . . . . . . . . 51

3.13 Reduction from CM 1-IN-3SAT to $C N_{d e c}^{\prime}\langle N, 2,4\rangle$. The edges connecting variable gadgets to clause gadgets belong in the area with the

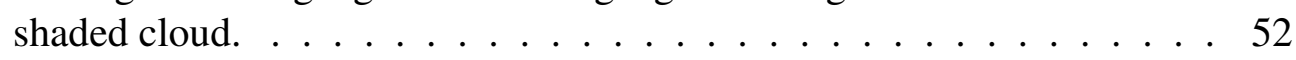

3.14 Gadgets used to represent variables and clauses. . . . . . . . . . . 53 
4.1 Small example to show that the transitivity constraint for the IP is necessary. . . . . . . . . . . . . . . . . . . 59

4.2 A DAG which obtains a factor 3 approximation. . . . . . . . . . . . 67

$4.3 \quad$ An optimal clustering and a worst-case clustering . . . . . . . . . . . 68

6.1 Results for some variants of the disjoint clustering problem that we investigated. .................... 87 


\section{List of Tables}

$6.1 \quad$ Approximability and inapproximability results . . . . . . . . . . . 86 


\section{Chapter 1}

\section{Introduction}

In this dissertation, we consider the problem of disjoint clustering in combinatorial circuits for delay minimization $(\mathrm{CN})$. Combinatorial circuits implement Boolean functions and produce a unique output for every combination of input signals [Kos04]. The gates and their interconnections in the circuit represent implementations of one or more Boolean function(s). The Boolean functions are realized by the assignment of the gates to chips.

Due to manufacturing process requirements and capacity constraints, it is generally not possible to place all of the circuit elements in one chip. Consequently, the circuit is partitioned into clusters, where each cluster represents a chip in the overall circuit design. The circuit elements are assigned to clusters while satisfying specific design constraints (e.g., cluster capacity) [RW93]. However, such an assignment might cause some source to sink path(s) to cross multiple cluster boundaries. The resulting design may not be the most efficient implementation.

Gates and their interconnections usually have delays. The delays of the interconnections are determined by the way the circuit is clustered. Intra-cluster delays $d$ are associated with the interconnections between gates in the same cluster. Inter-cluster delays $D$ are associated with the interconnections between gates in different clusters. The 
delay along a path from an input to an output is the sum of the delays of the gates and interconnections on the respective path. The delay of the overall circuit, with respect to a specific clustering, is the maximum delay among all paths that connect an input to any output in the clustered-circuit.

For example, consider the directed path $P$ representing a simple combinatorial circuit in Figure 1.1(a), Let every vertex of $P$ be unweighted (i.e., the weight of each vertex is one) with a zero delay. Let the cluster capacity equal two, the intra-cluster delay $d=0$, and the inter-cluster delay $D$ be any positive integer, where $D \gg d$. Suppose that we wish to find a disjoint clustering of $P$ such that no cluster exceeds the cluster capacity, and the delay of $P$ is minimized. Observe that the clustering of $P$ in Figure 1.1(b), where every vertex of $P$ belongs to a singleton cluster, is in an inefficient clustering with delay $4 \cdot D$. However, notice that the clustering of $P$ shown in Figure 1.1(c) induces the minimum delay of $2 \cdot D$.

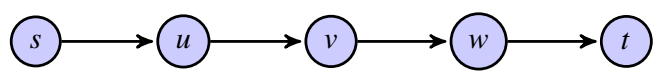

(a) Simple directed path $P$.

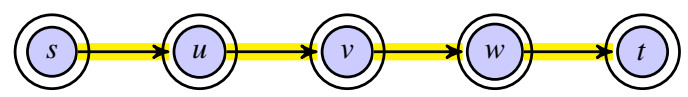

(b) An example of an inefficient clustering of $P$, where the delay is $4 \cdot D$.

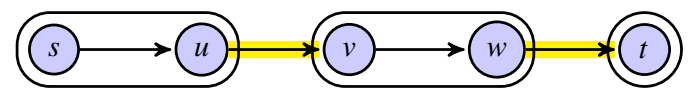

(c) An example of an optimal clustering of $P$, where the delay is $2 \cdot D$.

Figure 1.1: Simple example of clusterings of a directed path. 
Next, we consider another simple example of disjoint clustering of a combinatorial circuit represented by a directed acyclic graph (DAG) $G$ in Figure 1.2(a), Again, let every vertex of $G$ be unweighted with a zero delay. Let the cluster capacity equal two, the intra-cluster delay $d=0$, and the inter-cluster delay $D$ be any positive integer, where $D \gg d$. Suppose that we wish to find a disjoint clustering of $G$ such that no cluster exceeds the cluster capacity, and the delay of $G$ is minimized. Observe that the clustering of $G$ in Figure $1.2(\mathrm{~b})$, where every vertex of $G$ belongs to a singleton cluster, is in an inefficient clustering with delay $3 \cdot D$. The delay is not improved in the clustering of the DAG in Figure 1.2(c). However, notice that the clustering of $G$ shown in Figure 1.2(d) induces the minimum delay of $2 \cdot D$.

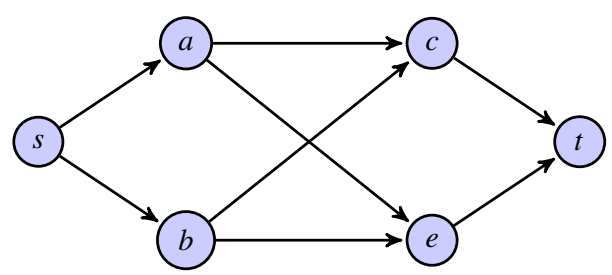

(a) An example of a DAG $G$.

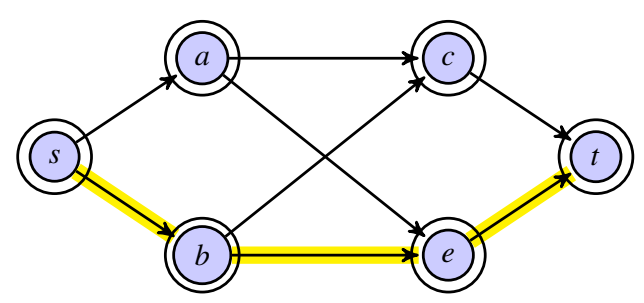

(b) Clustering with max delay $3 \cdot D$. 


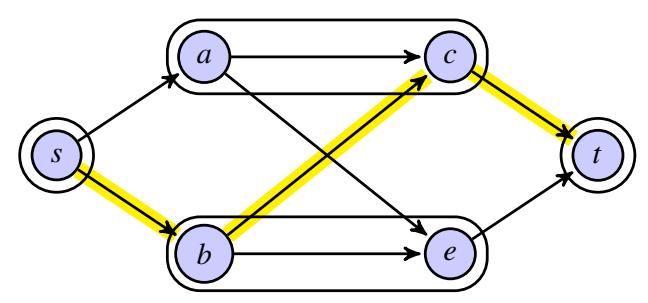

(c) Clustering with max delay $3 \cdot D$.

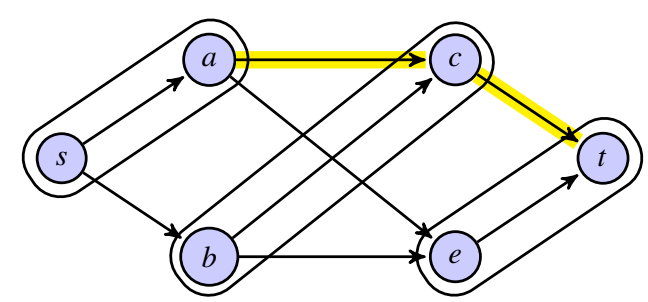

(d) Clustering with max delay $2 \cdot D$.

Although we did not enumerate all clusterings of the graphs shown in Figures 1.1(a) and $1.2(\mathrm{a})$, it can be quickly done. Therefore, finding the overall delay of each graph is not challenging in such small instances. However, it is easy to see that as the number of vertices increases, we encounter a combinatorial explosion when attempting to enumerate all possibilities.

\subsection{Clustering when replication is allowed}

The problem of clustering combinatorial circuits for delay minimization when logic replication is allowed (CA) is well-studied [LLT69, RW93]. It frequently arises in VLSI design. In CA, the goal is to find a clustering of a circuit that minimizes the delay of the overall circuit. CA has been shown to be solvable in polynomial time [LLT69, RW93]. However, when replication is allowed, circuit elements may be assigned to more than one cluster. 
Rajaraman and Wong [RW93] studied CA under the so-called general delay model in which the following holds:

1. each gate $v$ of the network has a delay given by $\delta(v)$,

2. no delay is encountered on an interconnection linking two gates in the same cluster, and

3. a delay of $D$ time units ( $D$ is a specified constant) is encountered on every interconnection linking two gates in different clusters.

In [RW93], they define a clustering as follows:

Definition 1.1.1. A clustering of a network $G=(V, E)$ is a triple $(H, \phi, \Sigma)$, where

1. $H=\left(V^{\prime}, E^{\prime}\right)$ is a directed acyclic graph

2. $\phi$ is a function mapping $V^{\prime}$ to $V$ such that

(a) for every edge $\left(u^{\prime}, v^{\prime}\right) \in E^{\prime},\left[\phi\left(u^{\prime}\right), \phi\left(v^{\prime}\right)\right] \in E$,

(b) for every node $v^{\prime} \in V^{\prime}$ and edge $\left[u, \phi\left(v^{\prime}\right)\right] \in E$, there exists a unique $u^{\prime} \in V^{\prime}$ such that $\phi\left(u^{\prime}\right)=u$ and $\left(u^{\prime}, v^{\prime}\right) \in E^{\prime}$, and

(c) for every $P O$ node $v \in V$, there exists a unique $v^{\prime} \in V^{\prime}$ such that $\phi\left(v^{\prime}\right)=v$, and

\section{3. $\Sigma$ is a partition of $V^{\prime}$.}

Using Definition 1.1.1, we proved the following two lemmas.

Lemma 1.1.1. The pre-image of an output is also an output.

Proof. Let $G=(V, E)$ have a clustering $(H, \phi, \Sigma)$, and by way of contradiction, suppose there exists a pre-image $v^{\prime} \in V(H)$ of an output $v \in V(G)$, such that $v^{\prime}$ is not also an output. This means $v^{\prime}$ has a successor in $H$, say $w^{\prime}$. By 2b of Definition 1.1.1, for every 
node $w^{\prime} \in V(H)$ and edge $\left[v, \phi\left(w^{\prime}\right)\right] \in E(G)$, there exists a unique $v^{\prime} \in V(H)$ such that $\phi\left(v^{\prime}\right)=v$ and $\left(v^{\prime}, w^{\prime}\right) \in E(H)$. But edge $\left[v, \phi\left(w^{\prime}\right)\right] \notin E(G)$, since $v$ is an output in $G$. So, there is exists no $v^{\prime} \in V(H)$ such that $\phi\left(v^{\prime}\right)=v$ and $\left(v^{\prime}, w^{\prime}\right) \in E(H)$. Therefore, $v$ 's pre-image $v^{\prime} \in V(H)$ must also be an output.

Lemma 1.1.2. Each path in a DAG G has a corresponding path of pre-images in a DAG $H$ (resulting from the replication of vertices of $G$ ), such that the length of the path of pre-images in $H$ is the same as the length of the path in $G$.

Proof. Let $G=(V, E)$ have a clustering $(H, \phi, \Sigma)$. Let $v_{1}, \ldots, v_{k}$ represent the vertices of some path in $G$, with length $k$ and endpoints $v_{1}$ and $v_{k}$, where $v_{k}$ is an output in $G$. By 2c of Definition 1.1.1, $v_{k}$ has a unique pre-image $v_{k}^{\prime} \in V(H)$; and from the previous result, we know that this unique pre-image $v_{k}^{\prime}$ is also an output. By $2 \mathrm{~b}$ of Definition 1.1.1. vertex $v_{k}^{\prime} \in V(H)$ and edge $\left[v_{k-1}, \phi\left(v_{k}^{\prime}\right)\right] \in E(G)$, implies there exists a unique $v_{k-1}^{\prime} \in V(H)$ such that $\phi\left(v_{k-1}^{\prime}\right)=v_{k-1}$ and $\left(v_{k-1}^{\prime}, v_{k}^{\prime}\right) \in E(H)$. Similarly, vertex $v_{k-1}^{\prime} \in$ $V(H)$ and edge $\left[v_{k-2}, \phi\left(v_{k-1}^{\prime}\right)\right] \in E(G)$, implies there exists a unique $v_{k-2}^{\prime} \in V(H)$ such that $\phi\left(v_{k-2}^{\prime}\right)=v_{k-2}$ and $\left(v_{k-2}^{\prime}, v_{k-1}^{\prime}\right) \in E(H)$. This argument continues until we reach vertex $v_{2}^{\prime} \in V(H)$ and edge $\left[v_{1}, \phi\left(v_{2}^{\prime}\right)\right] \in E(G)$, which implies there exists a unique $v_{1}^{\prime} \in V(H)$ such that $\phi\left(v_{1}^{\prime}\right)=v_{1}$ and $\left(v_{1}^{\prime}, v_{2}^{\prime}\right) \in E(H)$. Thus, there is a path of length $k$ in $H$ consisting of pre-images $v_{1}^{\prime}, \cdots, v_{k}^{\prime}$.

\subsubsection{Formulation of CA}

The precise formulation of CA as stated in [RW93] is as follows:

Given a combinational network $G=(V, E)$ with weight function $w: V \rightarrow \mathbb{R}^{+}$, weight capacity $M$, and delay function $\delta: V \mapsto \mathbb{R}^{+}$:

1. A clustering $\Gamma=(H, \phi, E)$ of $G$ is feasible if for every cluster $C \in E, W(C)$ is at most $M$. 
2. The circuit clustering problem (or CA as we call it) is to compute a feasible clustering $\Gamma$ of $G$ such that the delay of $\Gamma$ is minimum among all feasible clusterings of $G$.

\subsubsection{An example of $\mathrm{CA}$}

In [LLT69], the authors considered the example of a DAG shown in Figure 1.2. They describe a polynomial time algorithm that returns a clustering solution with replicated vertices. In this case, the vertices are unweighted with a zero delay, the cluster capacity is five, the intra-cluster delay $d=0$, and the inter-cluster delay $D=1$. Their algorithm does not purport to return a solution with the least amount of replication.

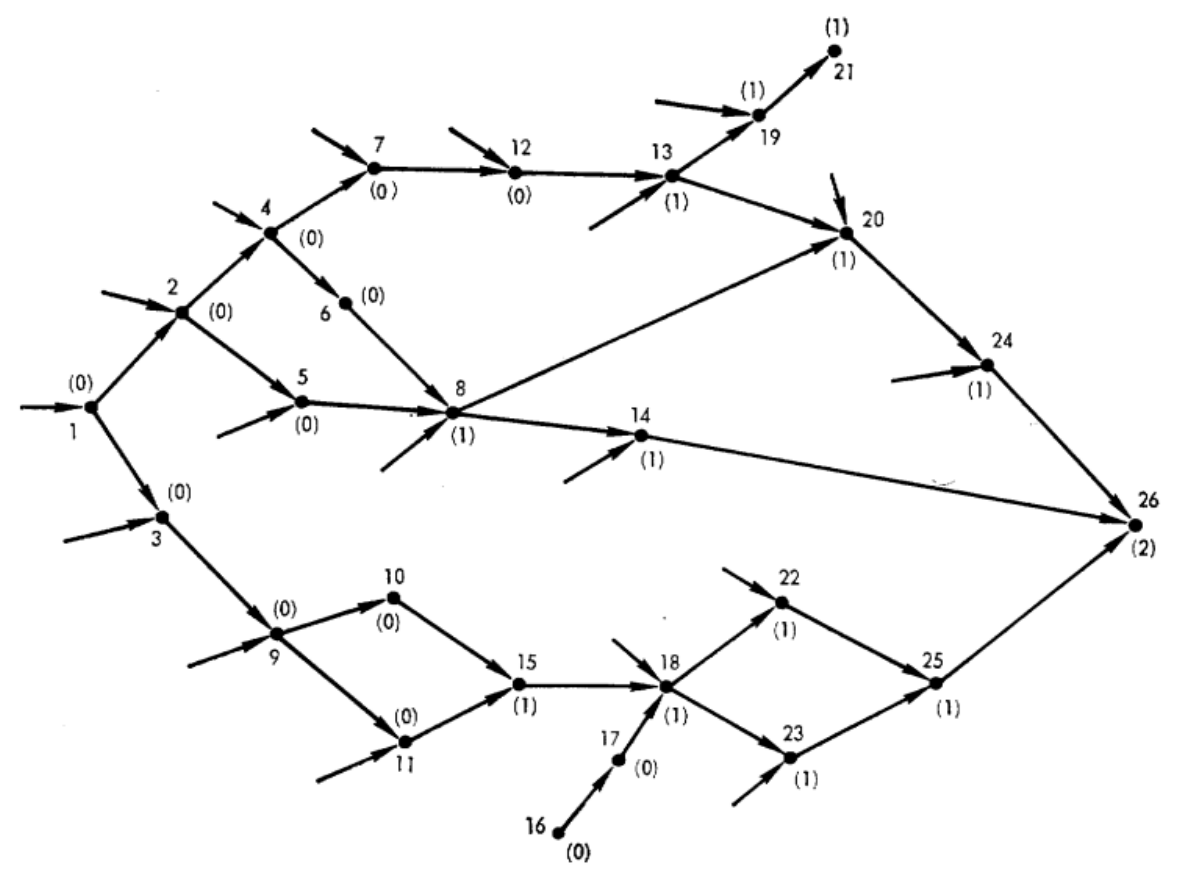

Figure 1.2: An instance of the clustering problem when replication of vertices is allowed.

The clustering of the DAG with replication of vertices shown in Figure 1.3(a) is a solution of the algorithm described in [LLT69]. Observe that 11 extra vertices that are 
introduced by the algorithm occur in the clustering solution (i.e., there are duplicates of some vertices in the original DAG).

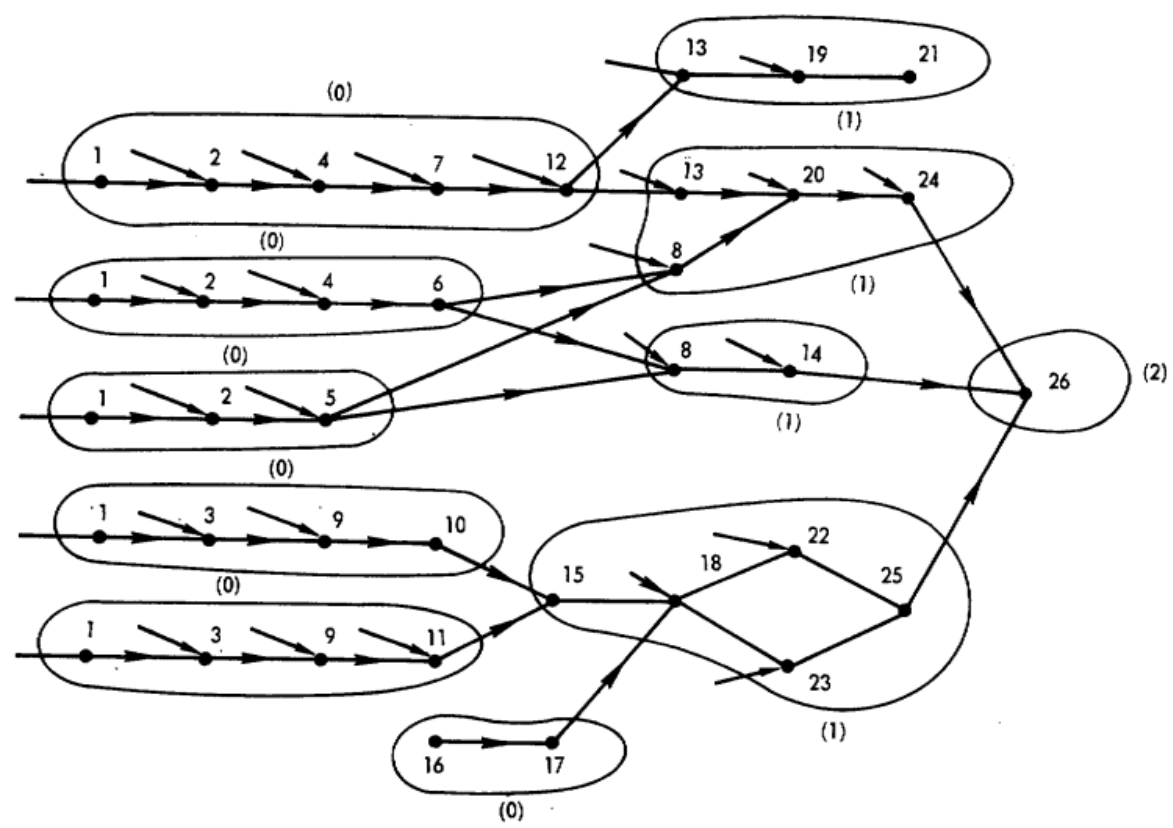

(a) An optimal clustering with replicated vertices.

Now, notice that the clustering of the DAG with replication shown in Figure 1.3(b) is an optimal clustering solution with fewer replicated vertices than that shown in Figure $1.3(\mathrm{a})$ 


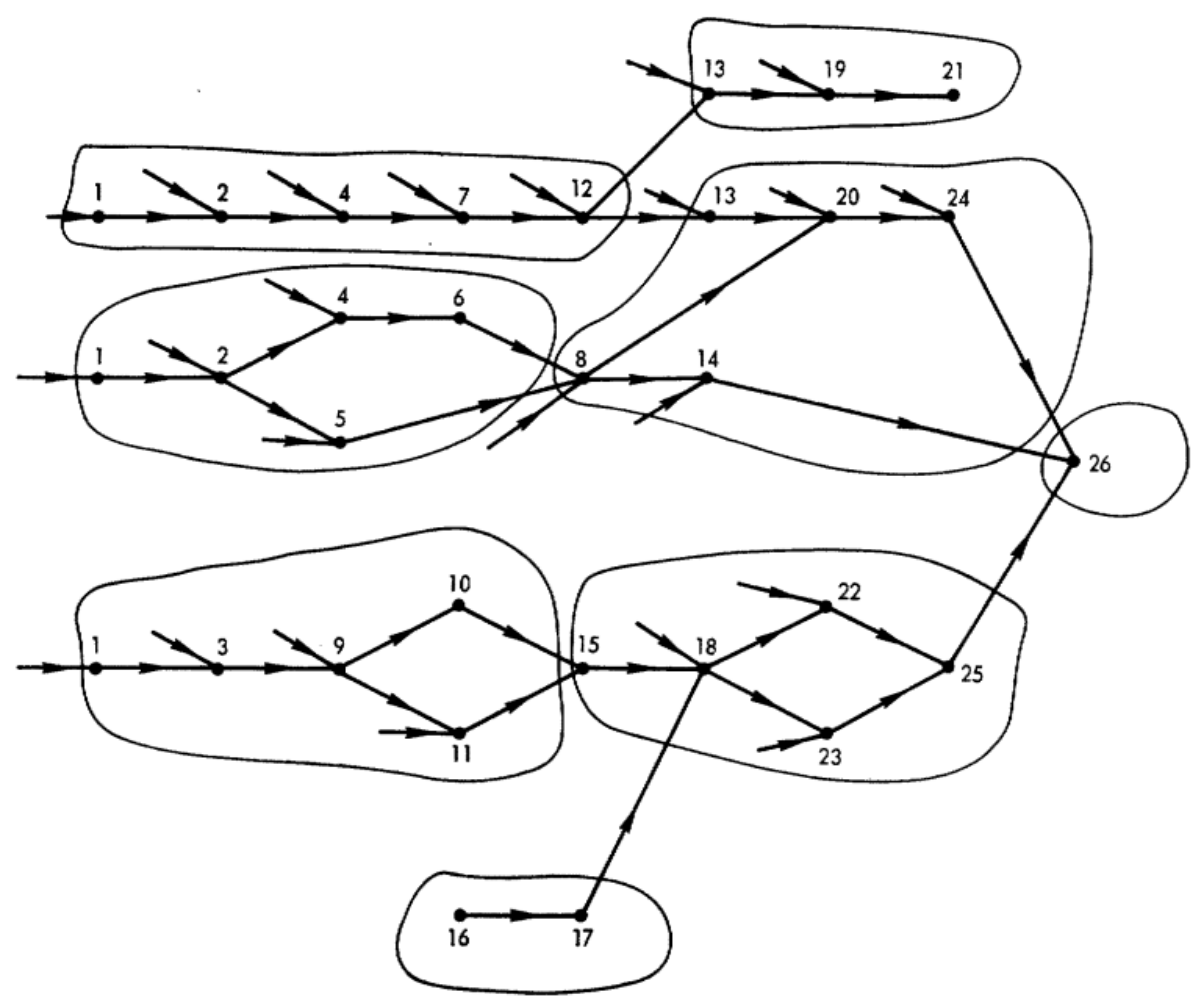

(b) An optimal clustering with fewer replicated vertices.

Note that unbounded replication can be quite expensive. As systems become increasingly more complex, the need for clustering without logic replication is crucial. It follows that $\mathrm{CN}$ is an important problem in VLSI design.

\subsection{Motivation and related work}

Lawler, Levitt, and Turner [LLT69] were the first to present an exact polynomial time algorithm for CA. They also show that in the special case when the undirected underlying graph is a tree, $\mathrm{CN}$ is polynomial time solvable. We refer to the model under which the problems were studied in [LLT69] as the "unit delay model" [MBSV91], where the delay $\delta(v)=0, \forall v \in V$ and the inter-cluster delay $D=1$. A more general delay model is presented by Murgai et al. [MBSV91], where $\delta(v) \geq 0, \forall v \in V$ and $D \geq$ 0. As per [RW93], this extension of the unit delay model is said to be more powerful 
and realistic. The algorithm for the general delay model proposed in [MBSV91] does not always return an optimal solution. However, they specify the conditions under which their algorithm returns an optimum.

Cong and Ding [CD94] also consider CA under the "unit delay model". However, instead of putting a constraint on the cluster capacity, they bound the number of incoming edges that are incident to a cluster (i.e., a "pin constrained variant of the CA problem). Such a variant of CA has applications in field-programmable gate array (FPGA) designs.

Rajaraman and Wong [RW93] consider CA under the more general delay model proposed in [MBSV91] and discuss a two-phase algorithm. The two phases include a labeling phase and a clustering phase, and the algorithm returns an optimal clustering in polynomial time.

Yang and Wong [YW97] expand on the work done in [RW93], and consider both area and pin constraints. Note that [MBSV91] and [RW93] consider area constraints only. In [YW97], they propose an efficient clustering algorithm that achieves an optimal solution under either the area constraint only or the pin constraint only. However, when both constraints are present, the algorithm does not always return the optimal solution. They specify the rare condition under which the algorithm fails to do so.

Cong and Romesis extend the study of CA to multi-level circuit clustering, with application to hierarchical field programmable gate array (FPGA) architecture, where clustering is applied recursively in two stages [CR01]. They show that the multi-level clustering problem for delay minimization with replication is NP-hard. They also propose an efficient heuristic for two-level clustering, providing a trade-off between area and delay by controlling the amount of replication.

Goldschmidt and Hochbaum [GH88] studied the multiway partition problem, where the goal is to find a partition of an edge-weighted graph $G$ into some non-empty clusters, such that the total edge weight between the clusters is minimum. This problem remains 
NP-hard, even when the input graph is unweighted, and there is no restriction on the cluster capacity. If the number of clusters is fixed (say $r$ ), then there is an algorithm that runs in time $O\left(n^{r^{2}}\right)$ that solves this restriction exactly. Here $n$ is the number of vertices of $G$. The case of the multiway partition problem, in which $r=2$, is frequently encountered in the literature. This case is called the bipartition problem. It is NP-hard for $k$-regular graphs [BCLS87], where $k \geq 3$ is a fixed constant. On the positive side, there is a dynamical programming based algorithm for solving this problem in the case of trees [BJ89, GM88, Mac88].

Mak and Wong [MW96] examine the amount of replication needed for clusterings that reduce the cut size. They focus on the bipartition problem with the goal of finding a minimum-cut that minimizes the size of the replication set. They present an efficient network-flow based algorithm that finds a min-cut partitioning which requires the least amount of replication to separate the gates of the circuit into two subsets. They also show how their algorithm applies to the problem of area-constrained min-cut partitioning with replication.

Since unbounded logic replication can be quite expensive, $\mathrm{CN}$ has been a focus of interest. Kagaris [ $\mathrm{Kag} 03]$ considered $\mathrm{CN}$ under area constraints and pin constraints, separately. Both area-constrained and pin-constrained problems were shown to be NPhard. Although not explicitly stated, the proof for the area-constrained problem established the NP-completeness of the decision version of a restriction of a variant of $\mathrm{CN}$ that we studied (cf. main theorem in [DGMS18]). They presented an efficient heuristic that makes use of the clustering algorithm described in [RW93]. Their experimental results showed that the delay is about 1.5 times the optimum (on average) for small inter-cluster delays, but increases with large inter-cluster delays and large cluster capacities. However, they did not establish provable bounds.

[SG11] and [SG14] explore the advantage of evolutionary algorithms aimed at reducing the delay and area in partitioning and floorplanning. In turn, this would reduce 
the wirelength. A hybrid of the evolutionary algorithms is used to find optimal solutions to VLSI physical design problems.

In [MWK15], the authors present an algorithm for simultaneous multilayer interconnect spacing. While satisfying maximum delay constraints, their unique algorithm guarantees to minimize the total dynamic power dissipation caused by an interconnect.

In [SHY $\left.{ }^{+} 10\right]$, adjustable delay buffers (ADBs) are used to minimize clock skew under different power modes. The ADBs have delays which can be tuned or adjusted. When the positions of some fixed number of ADBs are assumed to be predetermined, the authors propose a linear-time optimal algorithm. This algorithm assigns the values of the $\mathrm{ADBs}$ to minimize clock skew among all possible $\mathrm{ADB}$ assignments. In this case, there is a possibility of latency penalty. They also propose a modified algorithm to find an optimal solution with no latency penalty. Additionally, they give an efficient heuristic for finding good ADB positions.

Similar to [SHY $\left.{ }^{+} 10\right]$, the author of [Kao15] studies the use of ADBs to minimize clock skew under different power modes. To generate zero clock skew in a given clock tree, they start by assigning $\mathrm{ADB}$ positions. If the number of $\mathrm{ADBs}$ assigned do not meet the constraints of the previous solution, they use a bottom-up approach for removing ADBs to minimize clock skew while satisfying all constraints.

[SSA10] examines the methods used to solve bi-criterion VLSI circuit partitioning problems. The authors present a hybrid genetic algorithm (GA) which employs the Taguchi method for local search. They test their hybrid algorithm with a variety of benchmarks circuits and found it superior in comparison to the standard GA and tabu search algorithms reported in the literature.

A routability-driven clustering technique for area and power reduction in clustered FPGAs is presented in [SPMS02]. This technique uses a cell connectivity metric to identify seeds for efficient clustering. Effective seed selection, coupled with an interconnect-resource aware clustering and placement, can have a remarkable impact 
on circuit routability. It leads to better device utilization, reduction in power consumption and savings in area [SPMS02]. Additionally, the routing area is reduced by $35 \%$. The authors also show that their clustering technique can reduce the overall device power usage by an average of $13 \%$.

In [ML14], effective circuit partitioning techniques are employed by using clustering algorithms. The technique presented in [ML14] uses the circuit netlist to cluster the circuit in partitioning steps. It also minimizes the interconnection distance with the required iteration level. For the standard benchmark circuits the well-known clustering algorithms like $K$-Mean, $Y$-Mean, $K$-Medoid are performed. The results obtained in [ML14] show that the proposed techniques improve the delay. They also minimize the area by reducing the interconnection distance.

Aggarwal and Vitter [AV88] studied the complexity of sorting and other related problems under a two-level input/output (I/O) model (see Figure), where internal memory is limited, and there exists no bound (conceptually) on external storage. They obtained tight upper and lower bounds on the I/O communication required between internal mem-

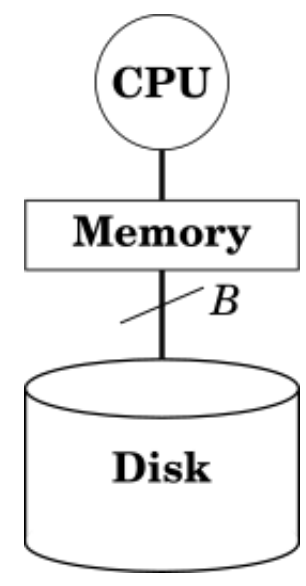
ory and external storage for the problems. For applications that store and process Figure 1.3: The I/O model [AB10]. vast and dynamically changing datasets, such communication can dramatically hinder performance [Vit98, Arg01]. Therefore, a great deal of attention is given to algorithms that seek to minimize the number of disk accesses used to solve a given problem [VV96, Vit01, MZ02, Vit06, AB10].

Asahiro et al. [AFIM06], established the computational complexity for a variant of 
the clustering problem proposed by Diwan et al. [DRSS96]. They called the variant Minimum Block Transfers with $B(\operatorname{MBT}(B))$ and considered the I/O model introduced in [AV88]. $\mathrm{MBT}(B)$ is similar to an unweighted variant of $\mathrm{CN}$ that we studied. We are given a DAG and a block size $B$ (i.e., a cluster capacity), the goal is to find a mapping of the nodes into blocks of size at most $B$ that minimizes the number of external arcs (i.e., inter-cluster edges) along any path from source to sink. They establish NP-hardness of MBT $(B)$ and obtain some bounds on inapproximability and approximability [AFIM06, AMY18].

The rest of this dissertation is organized as follows: The disjoint clustering problems that we study are formally described in Chapter 2. We provide some computational complexity results in Chapter 3 . We also show that our hardness results imply inapproximability below a certain threshold for several variants of CN. In Chapter 4 and 5, we propose some approximation and exact exponential algorithms, respectively. We conclude the dissertation with Chapter 6, by summarizing our main results and identifying avenues for future work. 


\section{Chapter 2}

\section{Statement of Problems}

In this chapter, we formally describe the problems studied in this dissertation. We start with graph preliminaries. Next, we formulate the main problem using the language of combinatorial circuits. Finally, we represent such circuits as directed acyclic graphs and formulate the main problem using graph-theoretic terminology.

\subsection{Graph preliminaries}

In this section, we define the main graph-theoretic concepts that are used in this dissertation.

Graphs considered in this dissertation do not contain loops or parallel edges. The degree of a vertex $v$ of an undirected graph $G$ is the number of edges of $G$ that are incident with $v$. The maximum degree of $G$ is denoted by $\Delta(G)$ or simply $\Delta$ when $G$ is known from the context.

A directed path (or, just a path) of a directed graph $G$ is a sequence $Q=$ $v_{0} e_{1} v_{1} \ldots e_{l} v_{l}$, where $v_{0}, v_{1}, \ldots, v_{l}$ are vertices of $G, e_{1}, \ldots, e_{l}$ are edges (also called arcs) of $G$, and $e_{j}=\left(v_{j-1}, v_{j}\right), 1 \leq j \leq l$. We call $l$ the length of the path $Q$, and sometimes we say that $Q$ is an $l$-path of $G$. If $v_{0}=v_{l}$, then $Q$ is called a directed cycle 
(or, just cycle). $G$ is said to be a directed acyclic graph (DAG), if it contains no directed cycles. For further terminology on graphs and directed graphs, one may consult [Wes01, BJG10].

A cluster is an arbitrary subset of the vertices of a DAG, and it does not have to be strongly connected. If $C$ is a cluster in a DAG $G$, then an edge is said to be a cut-edge if it connects a vertex of $C$ to a vertex from $V(G) \backslash C$. The degree of $C$ is the number of cut-edges incident with a vertex in $C$.

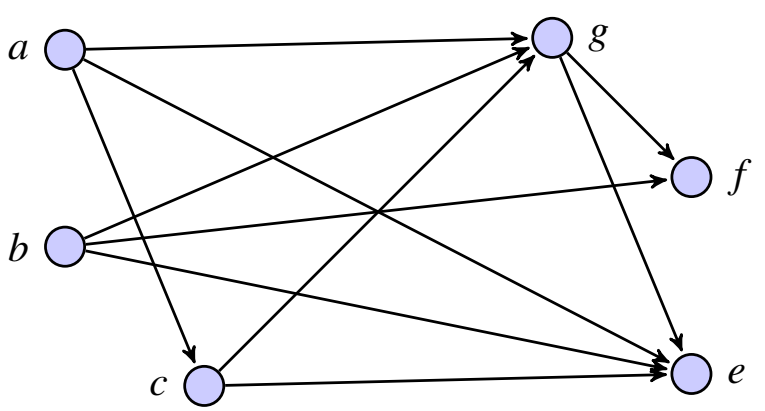

The indegree and outdegree of a verFigure 2.1: A DAG representing a combinatorial network with two sources and two sinks.

tex are the number of arcs that enter and leave the vertex, respectively. A source (sink, resp.) is a vertex with indegree zero (outdegree zero, resp.). It is well-known that every DAG has a source and a sink [BJG10]. Let $\mathscr{I}$ and $\mathscr{O}$ be the set of sources and sinks of $G$, respectively. Notice that $\mathscr{I}=\{a, b\}$ and $\mathscr{O}=\{e, f\}$ in the DAG of Figure 2.1, $C_{1}=\{a, c, g\}$ and $C_{2}=\{b, e, f\}$ represent a pair of disjoint clusters.

\subsection{Formulation of $\mathrm{CN}$ using combinatorial circuits}

A combinatorial circuit can be represented as a DAG $G=(V, E)$. In $G$, each vertex $v \in V$ represents a gate, and each edge $(u, v) \in E$ represents an interconnection between gates $u$ and $v$. In general, each gate in a circuit has an associated delay [MBSV91]. In the model that we consider in this dissertation, each interconnection has one of the following types of delays: (1) an intra-cluster delay, $d$, when there is an interconnection between two gates in the same cluster, or (2) an inter-cluster delay, $D$, when there is an 
interconnection between two gates in different clusters.

The delay along a path from an input to an output is the sum of the delays of the gates and interconnections that lie on the path. The delay of the overall circuit is the maximum delay among all source to sink paths in the circuit.

A clustering partitions the circuit into disjoint subsets. A clustering algorithm tries to achieve one or both of the following goals, subject to one or more constraints:

(1) The delay minimization through the circuit [RW93].

(2) The minimization of the total number of cut-edges [HG95].

In this dissertation, we study $\mathrm{CN}$ under the delay model described as follows:

1. Associated with every gate $v$ of the circuit, there is a delay $\delta(v)$ and a size $w(v)$.

2. The delay of an interconnection between two gates within a single cluster is $d$.

3. The delay of an interconnection between two gates in different clusters is $D$, where $D \gg d$.

The size of a cluster is the sum of the sizes of the gates in the cluster. The precise formulation of $\mathrm{CN}$ is as follows:

Given a combinatorial circuit, with each gate having a size and a delay, maximum degree $\Delta$, intra- and inter-cluster delays $d$ and $D$, respectively, and a positive integer $M$ called cluster capacity, the goal is to partition the circuit into clusters such that

1. The size of each cluster is bounded by $M$,

2. The delay of the circuit is minimized. 


\subsection{Graph-theoretic formulation of $\mathrm{CN}$}

In the rest of the dissertation, we focus on a graph-theoretic formulation of $\mathrm{CN}$. Given a clustering of a combinatorial circuit represented as a DAG $G=(V, E)$, the delays on the interconnections between gates induce an edge-delay function $\delta: E \rightarrow$ $\{d, D\}$ of $G$. The weight of a cluster is the sum of the weights of the vertices in the cluster. The delay-length of a directed path $P=v_{0} e_{1} v_{1} \ldots e_{l} v_{l}$ of $G$ is $\sum_{i=0}^{l} \delta\left(v_{i}\right)+$ $\sum_{i=1}^{l} \delta\left(e_{i}\right)$, where $\delta\left(e_{i}\right)$ is equal to $d$ if $v_{i-1}$ and $v_{i}$ are inside the same cluster, or $D$, otherwise.

We also employ the following notations and concepts. The symbol $X$ in our 3-tuple notation can be either $W$, which means that the vertices are weighted, or $N$, which means that the vertices are unweighted, the symbol $M$ is the cluster capacity, and $\Delta$ is the maximum number of arcs entering or leaving any vertex of the DAG (i.e., the maximum degree $\Delta$ of the underlying undirected graph of the DAG).

$\mathrm{CN}\langle X, M, \Delta\rangle$ is formulated (graph-theoretically) as follows: Given a $D A G G=$ $(V, E)$, with vertex-weight function $w: V \rightarrow \mathbb{N}$, delay function $\delta: V \rightarrow \mathbb{N}$, maximum degree $\Delta$, constants $d$ and $D$, and a cluster capacity $M$, the goal is to partition $V$ into clusters such that

1. The weight of each cluster is bounded by $M$,

2. The maximum delay-length of any path from a source to a sink of $G$ is minimized.

A clustering of $G$, such that the weight of each cluster is bounded by $M$, is called feasible. Given a feasible clustering of $G$, one can consider the corresponding edgelength function $\delta: E \rightarrow\{d, D\}$ of $G$. A clustering of $G$ is optimal if the maximum delay-length of any path from a source to a sink is the minimum among all clusterings.

In Figure 2.2, we consider a simple example of disjoint clustering in a combinatorial circuit represented by a DAG. In this example, the delays and weights of all vertices 

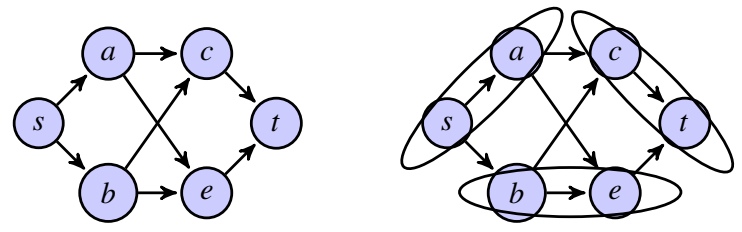

Figure 2.2: An example of a DAG and clustering of the DAG.

are equal to 0 and 1, respectively (i.e., $\delta(v)=0$ and $w(v)=1$ for all vertices $v$ in the DAG), the cluster capacity is $M=2$, the intra-cluster delay is $d=0$; and, the intercluster delay is $D=1$. It can be easily seen that the partition $\Sigma=\{\{s, a\},\{b, e\},\{c, t\}\}$ forms a feasible clustering such that the maximum delay-length of any path from $s$ to $t$ is 2. Moreover, we can quickly check to see that this clustering is optimal.

In this dissertation, we focus on a restriction of $\mathrm{CN}\langle X, M, \Delta\rangle$, when $\delta(v)=0$ for every vertex $v$ of $G$. Figure 2.3 shows a lattice of some of the variants of $\mathrm{CN}$ that we study. Observe that each node of the lattice is a variant of $C N\langle X, M, \Delta\rangle$, and each child in the lattice is a restriction of some parent(s).

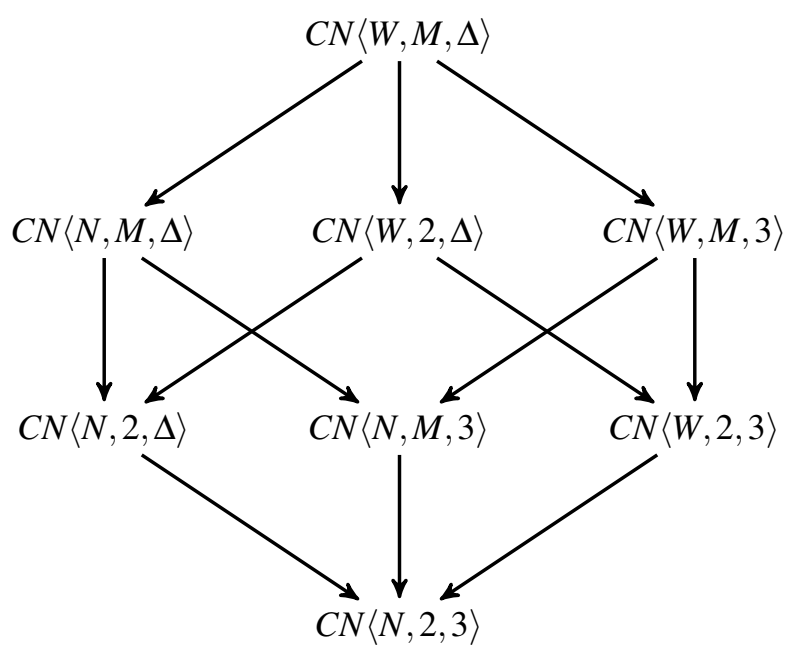

Figure 2.3: Some cases of the delay minimization problem that we investigate in this dissertation. 


\section{Chapter 3}

\section{Computational Complexity}

In this chapter, we establish the computational complexities of several variants of CN.

\subsection{Computational complexities of several variants of CN}

In this section, we obtain the main results that deal with the computational complexities of several variants of CN. We prove theorems that establish the NP-hardness of some variants. Moreover, our reductions imply that some variants of $\mathrm{CN}$ are inapproximable within a certain factor.

For many of our results, we consider $C N_{d e c}$, which is formulated as follows: Given a DAG $G=(V, E)$, with vertex-weight function $w: V \rightarrow \mathbb{N}$, delay function $\delta: V \rightarrow \mathbb{N}$, maximum degree $\Delta$, constants $d$ and $D$, cluster capacity $M$, and a positive integer $k$, decide whether we can partition $V$ into clusters such that

1. The weight of each cluster is bounded by $M$,

2. The maximum delay-length of any path from a source to a sink of $G$ is at most $k$. 
It is not hard to see that $C N_{d e c}$ is the decision version of $\mathrm{CN}\langle W, M, \Delta\rangle$. We make this correspondence explicit by writing $C N_{d e c}$ as $\mathrm{CN}_{d e c}\langle W, M, \Delta\rangle$. We use the same notation for restrictions of $\mathrm{CN}\langle W, M, \Delta\rangle$. If $A$ is a subset of positive integers, then $\mathrm{CN}_{d e c}\langle A, M, \Delta\rangle$ denotes the restriction of $\mathrm{CN}_{d e c}\langle W, M, \Delta\rangle$, when the weights of verticies of the input DAG are from $A$.

Our first theorem establishes the NP-completeness of $\mathrm{CN}_{d e c}\langle W, M, \Delta\rangle$. Clearly, this means that $\mathrm{CN}\langle W, M, \Delta\rangle$ is NP-hard.

Theorem 3.1.1. $C N_{d e c}\langle W, M, \Delta\rangle$ is NP-complete.

Proof. We recall $C N_{d e c}\langle W, M, \Delta\rangle$ as follows: Given a DAG $G=(V, E)$, with vertexweight function $w: V \rightarrow \mathbb{N}, \delta(v)=0 \forall v \in V$, maximum degree $\Delta$, constants $d$ and $D$, cluster capacity $M$, and a positive integer $k$, decide whether we can partition $V$ into clusters such that the weight of each cluster is bounded by $M$, and the maximum delaylength of any path from a source to a sink of $G$ is at most $k$.

It is clear that $\mathrm{CN}_{d e c}\langle W, M, \Delta\rangle$ is in NP since it follows from the well-known fact that finding a maximum weighted path in an edge-weighted DAG is polynomial time solvable [CLRS09].

In order to establish NP-hardness of $\mathrm{CN}_{d e c}\langle W, M, \Delta\rangle$, we present a reduction from PARTITION. For that purpose, we recall PARTITION as follows: Given a set $A=\left\{a_{1}, a_{2}, \ldots, a_{n}\right\}$, the goal is to check whether there is a set $A_{1} \subset A$, such that $\sum_{a_{i} \in A_{1}} a_{i}=\sum_{a_{i} \in A \backslash A_{1}} a_{i}$, where $i \in\{1,2, \ldots, n\}$. Without loss of generality, we assume that $\sum_{a_{i} \in A} a_{i}=B$ is even, otherwise the problem is trivial.

We now construct an instance $I^{\prime}$ of $\mathrm{CN}_{d e c}\langle W, M, \Delta\rangle$ as shown in Figure 3.1. There is a source $s$ connected to a sink $t$ through $n$ vertices labeled $u_{1}$ through $u_{n}$. Let $U$ denote the set of all vertices $u_{i}(1 \leq i \leq n)$. The vertices in $U$ are pairwise nonadjacent. Each vertex $u_{i} \in U$ has a weight $a_{i}$, and both $s$ and $t$ have weight $\frac{B}{2}$. We set $d=0$ and let $D$ be any positive integer. All vertices are given a delay of 0 . The cluster capacity is set to $B$, and we set $k=D$. The description of $I^{\prime}$ is complete. 


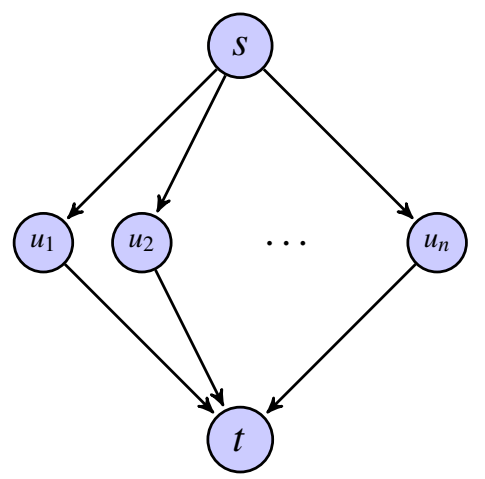

Figure 3.1: Reduction from PARTITION to $\mathrm{CN}_{d e c}\langle W, M, \Delta\rangle$.

Observe that $I^{\prime}$ can be constructed from an instance $I$ of PARTITION in polynomial time. In order to complete the proof of the theorem, we show that $I$ is a "yes" instance of PARTITION if and only if $I^{\prime}$ is a "yes" instance of $\mathrm{CN}_{d e c}\langle W, M, \Delta\rangle$.

Assume that $I$ is a "yes" instance of PARTITION. This means that there exists a partition of $A$ into $A_{1}$ and $A \backslash A_{1}$ such that $\sum_{a_{i} \in A_{1}} a_{i}=\sum_{a_{i} \in A \backslash A_{1}} a_{i}=\frac{B}{2}$. Group the vertices corresponding to the elements in $A_{1}$ with $s$, and the remaining vertices with $t$. Observe that the cluster capacity constraint is met. Moreover, the maximum delay-length of any path from $s$ to $t$ is $D$. This means that $I^{\prime}$ is a "yes" instance of $\mathrm{CN}_{d e c}\langle W, M, \Delta\rangle$.

For the proof of the converse statement, assume that $I^{\prime}$ is a "yes" instance of $\mathrm{CN}_{d e c}\langle W, M, \Delta\rangle$. This means that there is a way of partitioning the vertices of $G$ into clusters such that the maximum delay-length of any path from $s$ to $t$ is at most $D$. We observe that every vertex must be packed with either $s$ or $t$, otherwise the maximum delay-length must equal $2 \cdot D>k$. Let $U_{s}$ and $U_{t}$ denote the subsets of the vertices in $U$ that are packed with $s$ and $t$, respectively. Let $w(s)$ and $w(t)$ be the weights of vertices $s$ and $t$, respectively. Let $w\left(U_{s}\right)$ and $w\left(U_{t}\right)$ be the sums of the weights of the vertices in $U$ that are packed with $s$ and $t$, respectively. Clearly,

$$
w(s)+w\left(U_{s}\right)+w(t)+w\left(U_{t}\right)=2 \cdot B .
$$


Since

$$
w(s)+w\left(U_{s}\right) \leq B \text { and } w(t)+w\left(U_{t}\right) \leq B,
$$

we have

$$
w\left(U_{s}\right) \leq \frac{B}{2} \text { and } w\left(U_{t}\right) \leq \frac{B}{2}
$$

This implies that

$$
w\left(U_{s}\right)=\frac{B}{2} \text { and } w\left(U_{t}\right)=\frac{B}{2} .
$$

Thus, we have obtained the desired partition of $A$. Hence, $I$ is a "yes" instance of PARTITION.

The proof of Theorem 3.1.1 implies NP-hardness of $\mathrm{CN}\langle W, M, \Delta\rangle$, even for planar networks and therefore, strengthens the result in [Kag03]. Moreover, we obtain the following inapproximability result.

Corollary 3.1.1. $C N\langle W, M, \Delta\rangle$ does not admit a $(2-\varepsilon)$-approximation algorithm for each $\varepsilon>0$, unless $\mathbf{P}=\mathbf{N P}$.

Proof. By way of contradiction, suppose there exists a $(2-\varepsilon)$-approximation algorithm for $\mathrm{CN}_{d e c}\langle W, M, \Delta\rangle$. We construct a polynomial time algorithm for PARTITION as follows: 


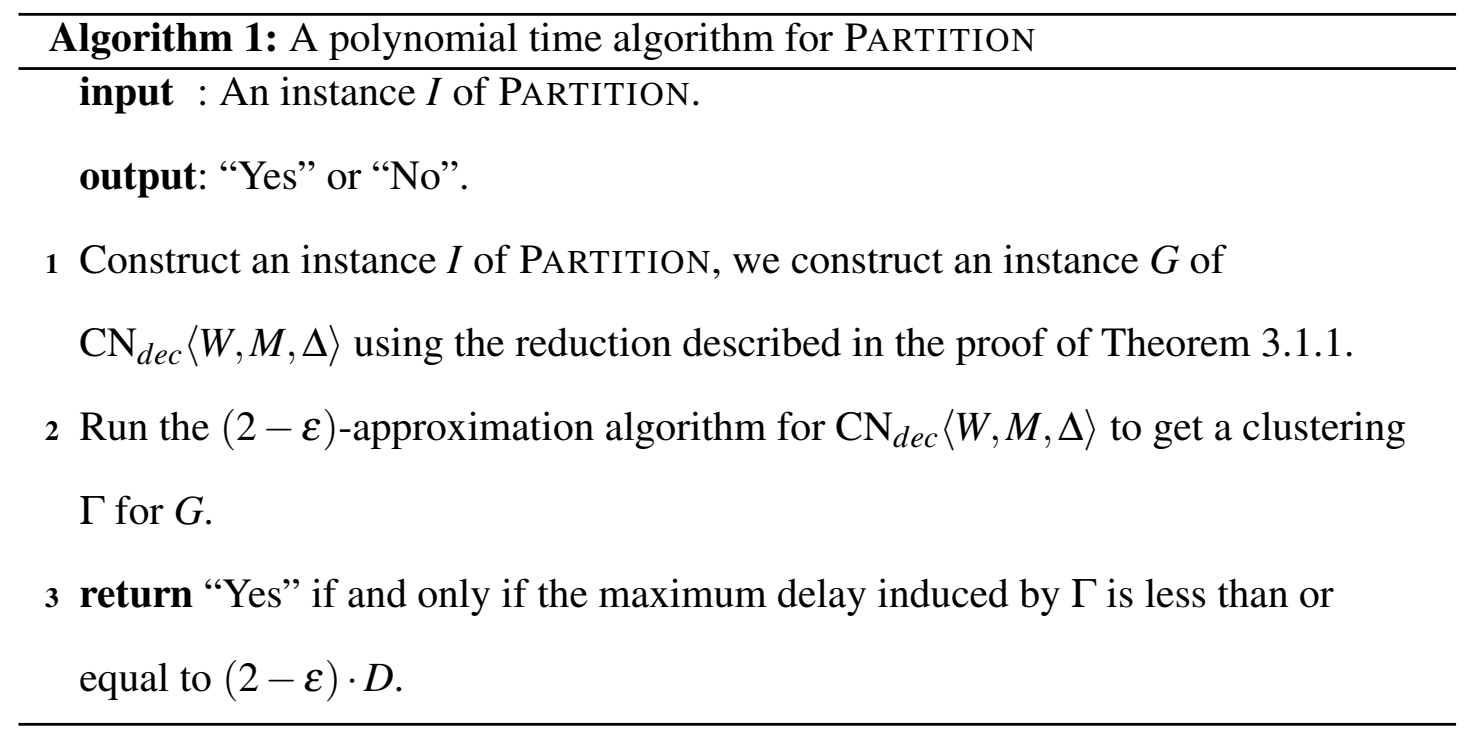

Let $O P T$ denote the delay of the optimal clustering of $G$. If $I$ is a "yes" instance of PARTITION, then $O P T \leq D$. Moreover, the maximum delay of any clustering solution of $G$ returned by the $(2-\varepsilon)$-approximation algorithm for $\mathrm{CN}_{d e c}\langle W, M, \Delta\rangle$ is at most $(2-\varepsilon) \cdot D$. Otherwise, the maximum delay of any clustering solution of $G$ must be at least $2 \cdot D$. Thus, the $(2-\varepsilon)$-approximation algorithm solves the instance $I$ of PARTITION exactly.

The next theorem serves to strengthen Theorem 3.1.1.

Theorem 3.1.2. $C N_{d e c}\langle W, M, 3\rangle$ is NP-complete. 


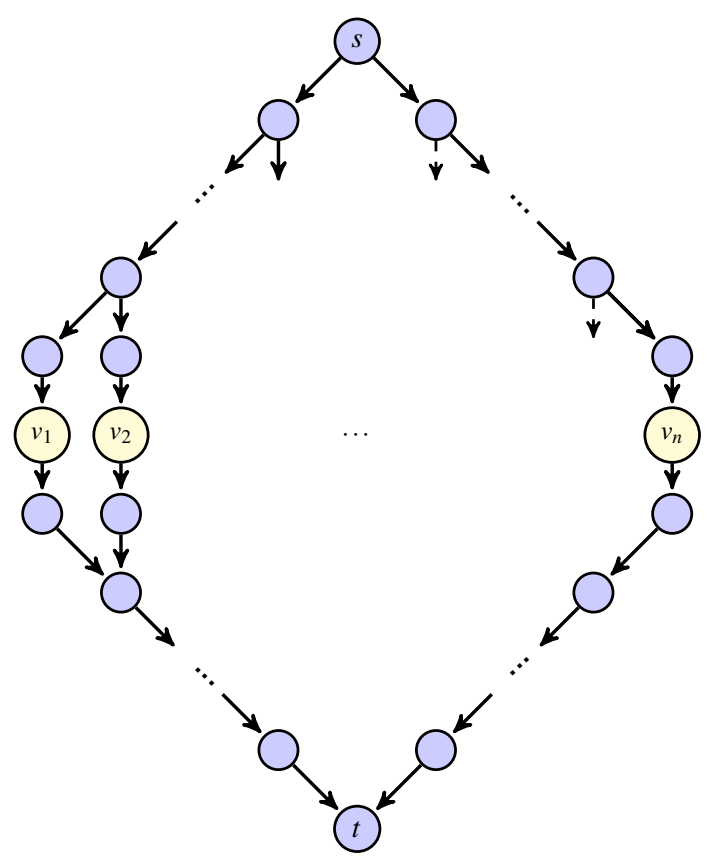

Figure 3.2: Reduction from PARTITION to $\mathrm{CN}_{d e c}\langle W, M, 3\rangle$.

Proof. We recall $C N_{d e c}\langle W, M, 3\rangle$ as follows: Given a DAG $G=(V, E)$, with vertexweight function $w: V \rightarrow \mathbb{N}, \delta(v)=0 \forall v \in V$, maximum degree $\Delta=3$, constants $d$ and $D$, cluster capacity $M$, and a positive integer $k$, decide whether we can partition $V$ into clusters such that the weight of each cluster is bounded by $M$, and the maximum delay-length of any path from a source to a sink of $G$ is at most $k$.

It is clear that $\mathrm{CN}_{d e c}\langle W, M, 3\rangle$ is in $\mathbf{N P}$ since it follows from the well-known fact that finding a maximum weighted path in an edge-weighted DAG is polynomial time solvable [CLRS09].

In order to establish NP-hardness of $\mathrm{CN}_{d e c}\langle W, M, 3\rangle$, we present a reduction from PARTITION. For that purpose, we recall PARTITION as follows: Given a set $A=\left\{a_{1}, a_{2}, \ldots, a_{n}\right\}$, the goal is to check whether there is a set $A_{1} \subset A$, such that $\sum_{a_{i} \in A_{1}} a_{i}=\sum_{a_{i} \in A \backslash A_{1}} a_{i}$, where $i \in\{1,2, \ldots, n\}$. Without loss of generality, we assume that $\sum_{a_{i} \in A} a_{i}=B$ is even, otherwise the problem is trivial.

We now construct an instance $I^{\prime}$ of $\mathrm{CN}_{d e c}\langle W, M, 3\rangle$ as shown in Figure 3.2. Let $U$ 
denote the set of all vertices $u_{i}(1 \leq i \leq n)$. The vertices in $U$ are pairwise nonadjacent. Each vertex $u_{i} \in U$ belongs to a distinct path that connects the source $s$ to the sink $t$. Let $S$ denote the set of all vertices that are predecessors of the vertices in $U$. Let $T$ denote the set of all vertices that are successors of the vertices in $U$. Note that in the underlying undirected graph, the subgraphs induced by $S$ and $T$ are isomorphic. Let $m$ denote the size of $S$ and $T$. Each vertex $u_{i} \in U$ has a weight of $a_{i}$. Every vertex in $S$ and $T$ has weight 1 . We set $d=0$ and let $D$ be any positive integer. Every vertex is given a delay of 0 . The cluster capacity $M$ is set to $\left(\frac{B}{2}+m\right)$, and we set $k=D$. The description of $I^{\prime}$ is complete.

Observe that $I^{\prime}$ can be constructed from an instance $I$ of PARTITION in polynomial time. In order to complete the proof of the theorem, we show that $I$ is a "yes" instance of PARTition if and only if $I^{\prime}$ is a "yes" instance of $\mathrm{CN}_{d e c}\langle W, M, 3\rangle$.

Assume that $I$ is a "yes" instance of PARTITION. This means that there exists a partition of $A$ into $A_{1}$ and $A \backslash A_{1}$ such that $\sum_{a_{i} \in S_{1}} a_{i}=\sum_{a_{i} \in A \backslash A_{1}} a_{i}=\frac{B}{2}$. Group the vertices corresponding to the elements in $A_{1}$ with $S$, and the remaining vertices with $T$. Observe that the cluster capacity constraint is met. Moreover, the maximum delaylength of any path from the source $s$ to the $\operatorname{sink} t$ is $D$. This means that $I^{\prime}$ is a "yes" instance of $\mathrm{CN}_{d e c}\langle W, M, 3\rangle$.

Conversely, assume that $I^{\prime}$ is a "yes" instance of $\mathrm{CN}_{d e c}\langle W, M, 3\rangle$. This means that there is a way of partitioning the vertices of the DAG in Figure 3.2 into clusters, such that the cluster capacity constraint is satisfied, and the maximum delay-length of any path from $s$ to $t$ is at most $D$. Since $S$ and $T$ have the same underlying structure and $|S|=|T|$, then without loss of generality, we may assume that every vertex in $S$ is clustered together, and every vertex in $T$ is clustered together. Furthermore, each vertex $u_{i} \in U$ must be clustered with the vertices in either $S$ or $T$. Otherwise, the delay-length of the path from $s$ to $t$ is strictly greater than $D$. Let $U_{S}$ and $U_{T}$ denote the subsets of the vertices in $U$ that are packed with $S$ and $T$, respectively. Observe that $U_{S} \cup U_{T}=U$. 
Notice that the delay-length of any path from $s$ to a vertex in $U_{S}$ is 0 , and the delaylength of any path from a vertex in $U_{T}$ to $t$ is also 0 . Let $w(S)$ and $w(T)$ denote the sum of the weights of all vertices in $S$ and $T$, respectively. Notice that $w(S)=w(T)=m$. Let $w\left(U_{S}\right)$ and $w\left(U_{T}\right)$ denote the sum of the weights of all vertices in $U_{S}$ and $U_{T}$, respectively. Clearly,

$$
w\left(U_{S}\right)+w\left(U_{T}\right)+w(S)+w(T)=B+2 \cdot m .
$$

Since

$$
w\left(U_{S}\right)+w(S) \leq\left(\frac{B}{2}+m\right) \text { and } w\left(U_{T}\right)+w(T) \leq\left(\frac{B}{2}+m\right)
$$

then

$$
w\left(U_{S}\right) \leq \frac{B}{2} \text { and } w\left(U_{T}\right) \leq \frac{B}{2}
$$

This implies that

$$
w\left(U_{S}\right)=\frac{B}{2} \text { and } w\left(U_{T}\right)=\frac{B}{2} .
$$

Thus, we have obtained the desired partition of $A$. Hence, $I$ is a "yes" instance of PARTITION.

The proof of Theorem 3.1.2 implies an inapproximability result for $\mathrm{CN}\langle W, M, 3\rangle$.

Corollary 3.1.2. $C N\langle W, M, 3\rangle$ does not admit a $(2-\varepsilon)$-approximation algorithm for each $\varepsilon>0$, unless $\mathbf{P}=\mathbf{N P}$.

Proof. By way of contradiction, suppose there exists a $(2-\varepsilon)$-approximation algorithm for $\mathrm{CN}_{d e c}\langle W, M, 3\rangle$. We construct a polynomial time algorithm for PARTITION as follows: 


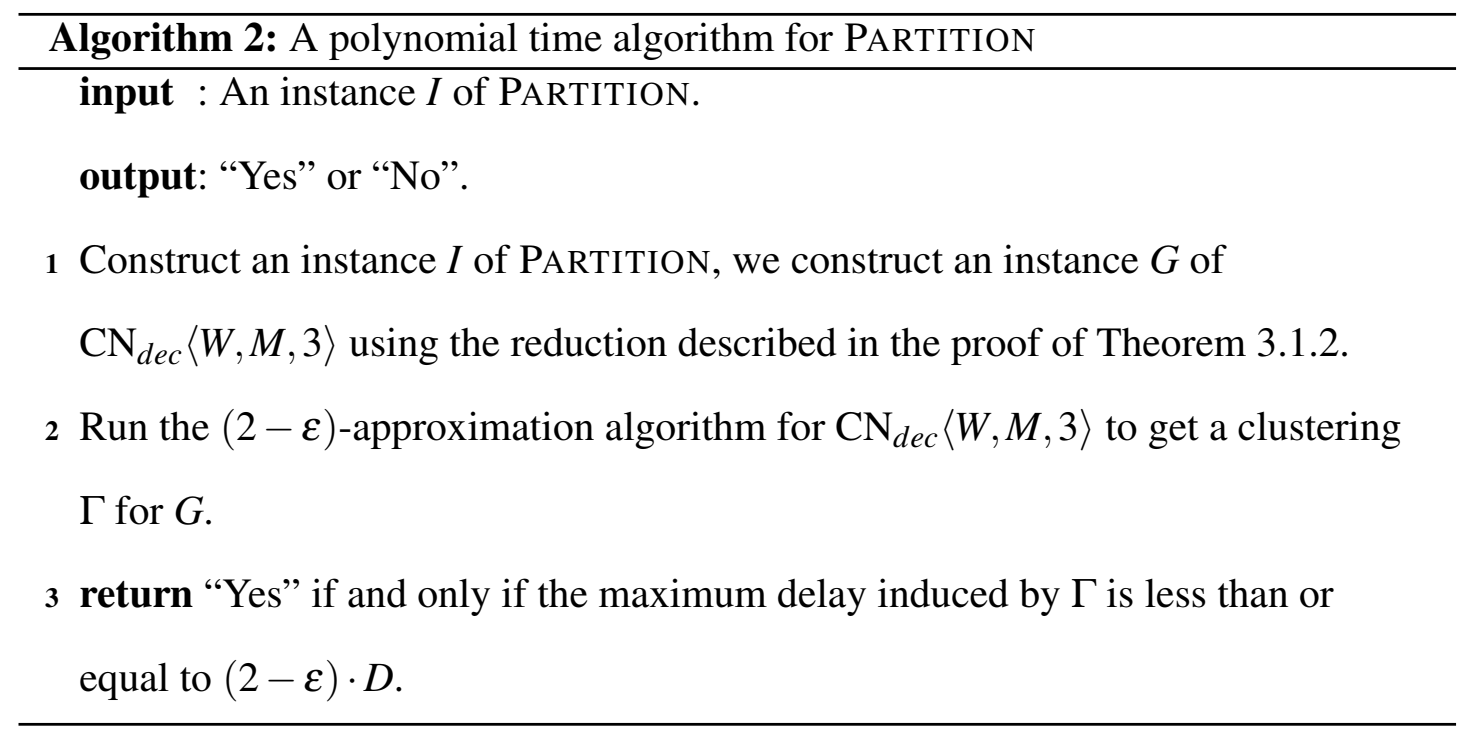

Let $O P T$ denote the delay of the optimal clustering of $G$. If $I$ is a "yes" instance of PARTITION, then $O P T \leq D$. Moreover, the maximum delay of any clustering solution of $G$ returned by the $(2-\varepsilon)$-approximation algorithm for $\mathrm{CN}_{d e c}\langle W, M, 3\rangle$ is at most $(2-\varepsilon) \cdot D$. Otherwise, the maximum delay of any clustering solution of $G$ must be at least $2 \cdot D$. Thus, the $(2-\varepsilon)$-approximation algorithm solves the instance $I$ of PARTITION exactly.

The next theorem implies NP-hardness of $\mathrm{CN}\langle[3], 3,3\rangle$. In the proof, we use a 3SAT reduction modeled after the one presented in [Kag03].

Theorem 3.1.3. $C N_{d e c}\langle[3], 3,3\rangle$ is NP-complete.

Proof. We recall $C N_{d e c}\langle[3], 3,3\rangle$ as follows: Given a DAG $G=(V, E)$, with vertexweight function $w: V \rightarrow\{1,2,3\}, \delta(v)=0 \forall v \in V$, maximum degree $\Delta=3$, constants $d$ and $D$, cluster capacity $M=3$, and a positive integer $k$, decide whether we can partition $V$ into clusters such that the weight of each cluster is bounded by $M$, and the maximum delay-length of any path from a source to a sink of $G$ is at most $k$.

It is clear that $\mathrm{CN}_{d e c}\langle[3], 3,3\rangle$ is in $\mathbf{N P}$ since it follows from the well-known fact that finding a maximum weighted path in an edge-weighted DAG is polynomial time 
solvable [CLRS09].

In order to establish NP-hardness of $\mathrm{CN}_{d e c}\langle[3], 3,3\rangle$, we present a reduction from 3SAT. For that purpose, we recall 3SAT as follows: Given a 3-CNF formula $\phi$ with $n$ variables $x_{1}, \ldots, x_{n}$ and $m$ clauses $C_{1}, \ldots, C_{m}$, the goal is to check whether $\phi$ has a satisfying assignment. Without loss of generality, for all $i \in\{1, \ldots, n\}$ we assume that each variable $x_{i}$ in $\phi$ appears at most three times and each literal at most twice. (Any 3SAT instance can be transformed to satisfy these properties in polynomial time [Pap94].)

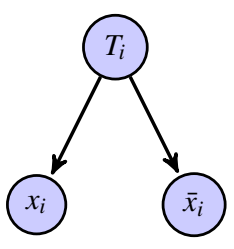

(a) Variable

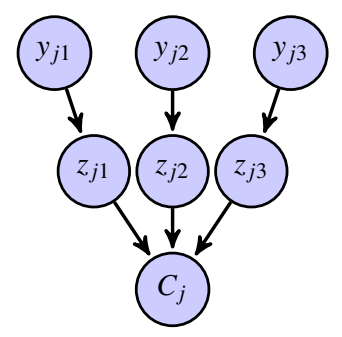

(b) Clause

Figure 3.3: Gadgets used to represent variables and clauses.

Let each variable $x_{i}(1 \leq i \leq n)$ be represented by a variable gadget as shown in Figure 3.3(a), Let each clause $C_{j}(1 \leq j \leq m)$ be represented by a clause gadget as shown in Figure 3.3(b), If a variable $x_{i}$ or its complement $\bar{x}_{i}$ is the $1 \mathrm{st}$, 2nd, or 3rd literal of a clause $C_{j}$, then the corresponding vertex labeled $x_{i}$ (or $\left.\bar{x}_{i}\right)$ is connected to a sink labeled $C_{j}$ through a pair of vertices labeled $\left\{y_{j 1}, z_{j 1}\right\},\left\{y_{j 2}, z_{j 2}\right\}$, or $\left\{y_{j 3}, z_{j 3}\right\}$, respectively. A simple example of the construction of an instance $I^{\prime}$ is shown in Figure 3.4. where $\phi=C_{1} \wedge C_{2}$, with $C_{1}=\left(x_{1}, \bar{x}_{2}, x_{3}\right)$ and $C_{2}=\left(x_{2}, x_{3}, x_{4}\right)$.

We now construct an instance $I^{\prime}$ of $\mathrm{CN}_{d e c}\langle[3], 3,3\rangle$ as shown in Figure 3.5. The resulting DAG $G$ represents a combinatorial circuit. Let $U$ denote the set of all vertices labeled $x_{i}$ or $\bar{x}_{i}(1 \leq i \leq n)$. There are $n$ sources labeled $T_{i}(1 \leq i \leq n)$ and $m$ sinks labeled $C_{j}(1 \leq j \leq m)$. They are connected through some vertices in $U$ and $3 \cdot m$ pairs of vertices labeled $\left\{y_{j p}, z_{j p}\right\}(1 \leq j \leq m, 1 \leq p \leq 3)$. Each $y_{j p}$ is connected to exactly 


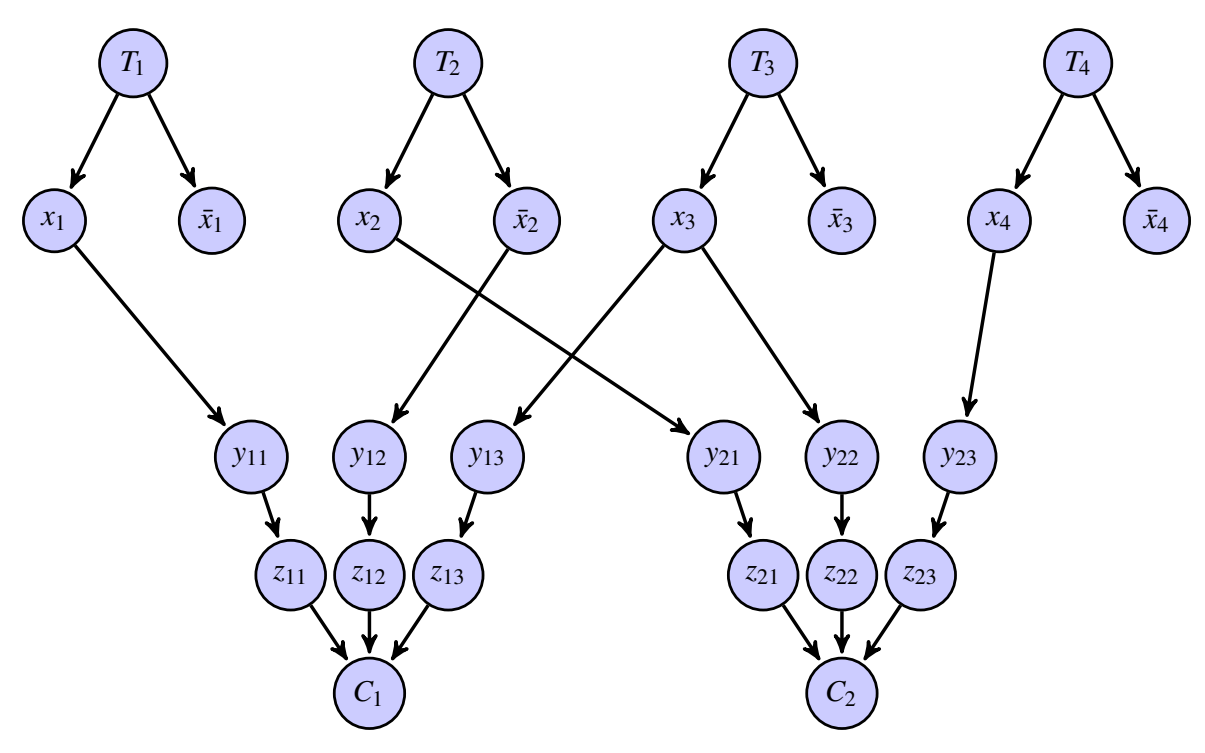

Figure 3.4: Simple example

one variable gadget. For every $j$, no two vertices in the set $\left\{y_{j 1}, y_{j 2}, y_{j 3}\right\}$ are adjacent to both $x_{i}$ and $\bar{x}_{i}$ of the same variable gadget. In other words, $x_{i}$ and $\bar{x}_{i}$ cannot both be connected to the same clause gadget. Every $T_{i}, z_{j p}$, and $C_{j}$ has a weight of 1 , every $x_{i}, \bar{x}_{i} \in U$ has a weight of 2 , and every $y_{j p}$ has a weight of 3 . We set $d=0$ and let $D$ be any positive integer. All vertices are given a delay of 0 . The cluster capacity $M$ is set to 3 , and we set $k=3 \cdot D$. The description of $I^{\prime}$ is complete.

Observe that $I^{\prime}$ can be constructed from $I$ in polynomial time. In order to complete the proof of the theorem, we show that $I$ is a "yes" instance of 3SAT if and only if $I^{\prime}$ is a "yes" instance of $\mathrm{CN}_{d e c}\langle[3], 3,3\rangle$.

Suppose that $I$ is a "yes" instance of $3 \mathrm{SAT}$. This means that there exists an assignment of $\phi$ such that every clause has at least one true literal. If a literal $x_{i}$ is set to true, then the corresponding vertex $x_{i}$ (or $\bar{x}_{i}$ ) is clustered with $T_{i}$. However, if a literal $x_{i}$ is set to false, then the corresponding vertex $x_{i}$ is clustered alone. Since $M=3$, every $y_{j p}$ must be clustered alone. Since each clause $C_{j}$ has at least one true literal, the vertex $z_{j p}$ along the path of the vertex $x_{i}$ (or $\bar{x}_{i}$ ) corresponding to that true literal is clustered alone. The resulting delay-length of the corresponding source to sink path is $3 \cdot D$. It 

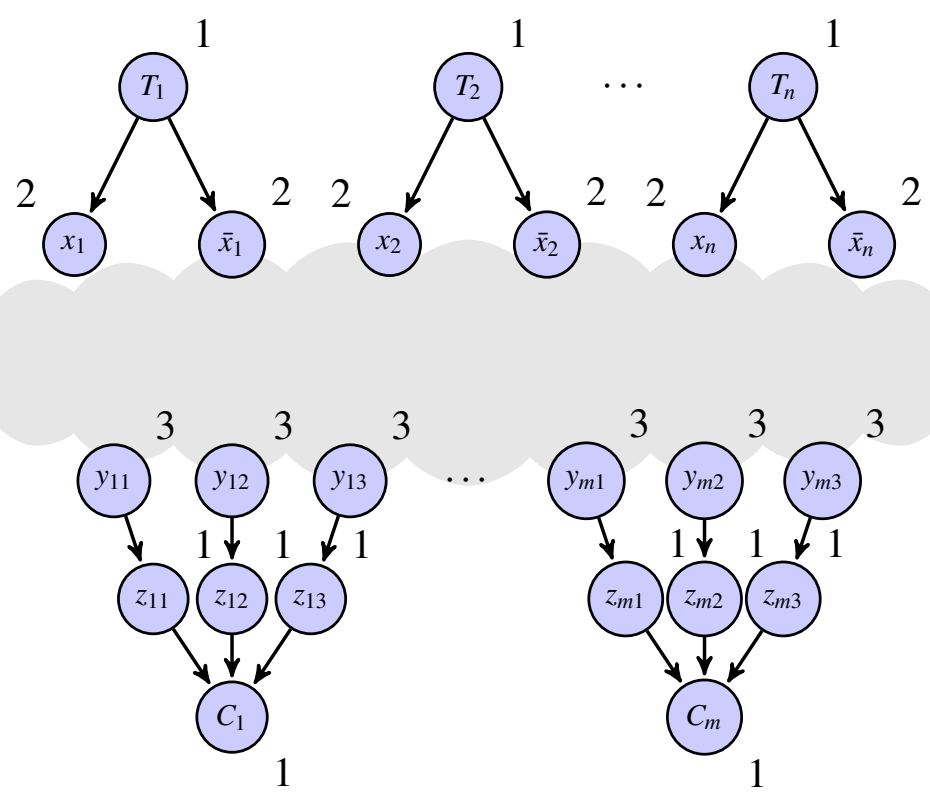

Figure 3.5: Reduction from the 3SAT problem to $\mathrm{CN}_{d e c}\langle[3], 3,3\rangle$. The edges connecting variable gadgets to clause gadgets belong in the area with the shaded cloud.

is safe to cluster the remaining two $z_{j p}$ vertices with $C_{j}$, even if they both belong to paths corresponding to true literals. In this case, the resulting paths have delay-length $2 \cdot D<3 \cdot D=k$. However, if either one these two $z_{j p}$ vertices belongs to a path corresponding to a false literal, then it must be clustered with $C_{j}$ to avoid exceeding the bound on the delay-length. Observe that the cluster capacity constraint is satisfied, and the maximum delay-length of any path from a source $T_{i}$ to a $\operatorname{sink} C_{j}$ is $3 \cdot D$. This means that $I^{\prime}$ is a "yes" instance of $\mathrm{CN}_{d e c}\langle[3], 3,3\rangle$.

Conversely, suppose that $I^{\prime}$ is a "yes" instance of $\mathrm{CN}_{d e c}\langle[3], 3,3\rangle$. This means that there is a way of partitioning the vertices of $G$ into clusters of capacity $M=3$, such that the maximum delay-length of any path from a source to a sink is at most $3 \cdot D$.

Since $M=3$, again notice that every $y_{j p}$ must be clustered alone. Each $\operatorname{sink} C_{j}$ may be clustered with at most two vertices. This means that at least one $z_{j p}$ is clustered alone. Consider the vertex $x_{i}$ (or $\bar{x}_{i}$ ) along a path corresponding to a $z_{j p}$ clustered alone. Since any source to sink path with a $z_{j p}$ clustered alone has a delay-length of at least 
$3 \cdot D$, then $T_{i}$ must be clustered with vertex $x_{i}$ (or $\bar{x}_{i}$ ). Otherwise, the delay-length of the path would be $4 \cdot D>3 \cdot D=k$. Furthermore, since the cluster capacity is satisfied, either $x_{i}$ or $\bar{x}_{i}$ (but not both) can be clustered with $T_{i}$. Take each literal that corresponds to a vertex $x_{i}$ clustered with $T_{i}$, and set its value to true. Now, notice that any $z_{j p}$ along a path in which $x_{i}$ (or $\bar{x}_{i}$ ) is clustered alone, must be clustered with the $\operatorname{sink} C_{j}$. Otherwise, the delay-length of the path would be $4 \cdot D>3 \cdot D=k$. Take each literal that corresponds to a vertex $x_{i}$ not clustered with $T_{i}$ and set its value to false. Notice that at least one true literal appears in every clause. Thus, a satisfying clustering for $G$ yields a satisfying assignment for $\phi$. Hence, $I$ is a "yes" instance of 3SAT.

The proof of Theorem 3.1.3 implies an inapproximability result for $\mathrm{CN}\langle[3], 3,3\rangle$.

Corollary 3.1.3. $C N\langle[3], 3,3\rangle$ does not admit a $\left(\frac{4}{3}-\varepsilon\right)$-approximation algorithm for any $\varepsilon>0$, unless $\mathbf{P}=\mathbf{N P}$.

Proof. By way of contradiction, suppose there exists a $\left(\frac{4}{3}-\varepsilon\right)$-approximation algorithm for $\mathrm{CN}_{d e c}\langle[3], 3,3\rangle$. We construct a polynomial time algorithm for $3 \mathrm{SAT}$ as follows:

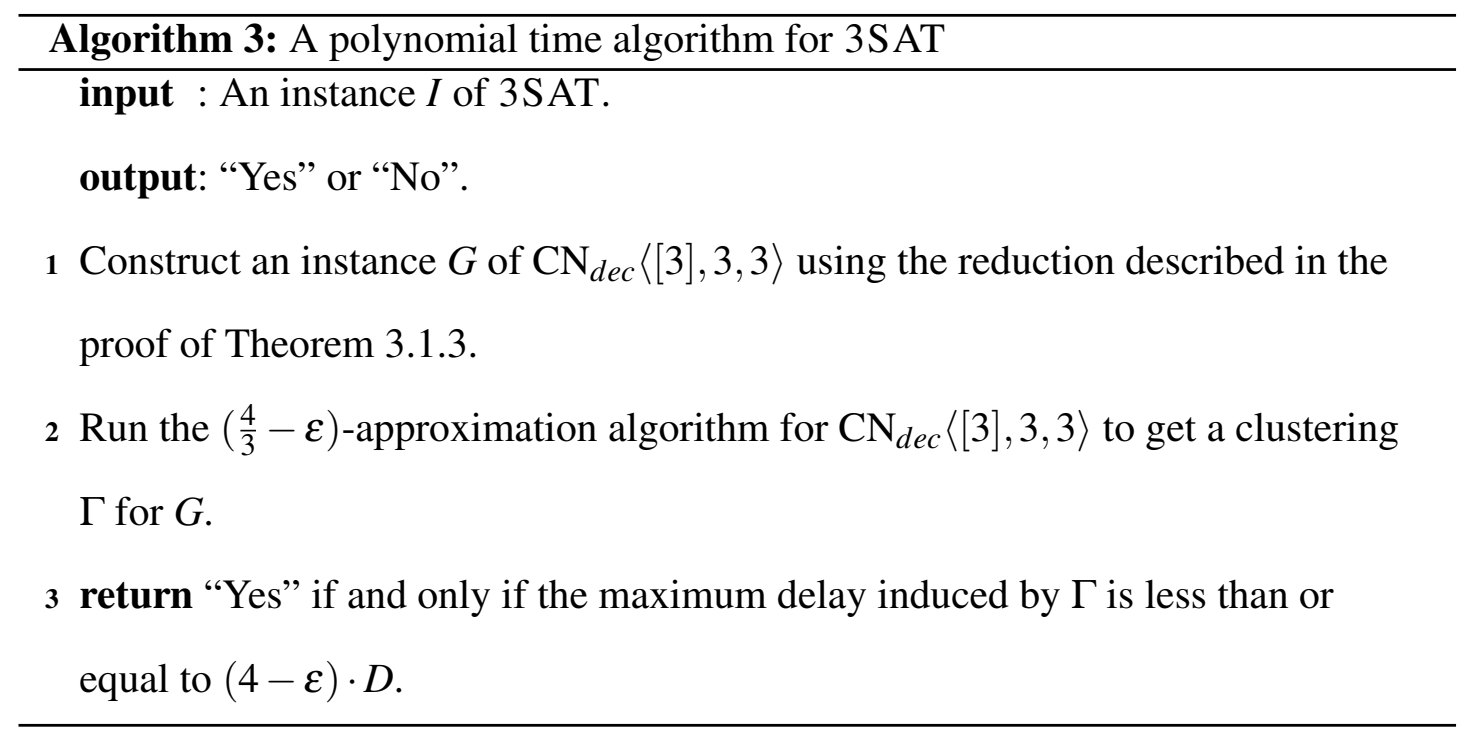


Let $O P T$ denote the delay of the optimal clustering of $G$. If $I$ is a "yes" instance of $3 \mathrm{SAT}$, then $O P T \leq 3 \cdot D$. Moreover, the maximum delay of any clustering solution of $G$ returned by the $\left(\frac{4}{3}-\varepsilon\right)$-approximation algorithm for $\mathrm{CN}_{d e c}\langle[3], 3,3\rangle$ is at most $(4-\varepsilon) \cdot D$. Otherwise, the maximum delay of any clustering solution of $G$ must be at least $4 \cdot D$. Thus, the $\left(\frac{4}{3}-\varepsilon\right)$-approximation algorithm solves the instance $I$ of $3 \mathrm{SAT}$ exactly.

The next theorem implies NP-hardness of $\mathrm{CN}\langle[2], 2,4\rangle-$ a restriction of $\mathrm{CN}\langle W, 2, \Delta\rangle$.

Theorem 3.1.4. $C N_{d e c}\langle[2], 2,4\rangle$ is NP-complete.

Proof. We recall $C N_{d e c}\langle[2], 2,4\rangle$ as follows: Given a DAG $G=(V, E)$, with vertexweight function $w: V \rightarrow\{1,2\}, \delta(v)=0 \forall v \in V$, maximum degree $\Delta=4$, constants $d$ and $D$, cluster capacity $M=2$, and a positive integer $k$, decide whether we can partition $V$ into clusters such that the weight of each cluster is bounded by $M$, and the maximum delay-length of any path from a source to a sink of $G$ is at most $k$.

It is clear that $\mathrm{CN}_{d e c}\langle[2], 2,4\rangle$ is in NP since it follows from the well-known fact that finding a maximum weighted path in an edge-weighted DAG is polynomial time solvable [CLRS09].

In order to establish NP-hardness of $\mathrm{CN}_{d e c}\langle[2], 2,4\rangle$, we present a reduction from 3-Bounded Positive 1-IN-3SAT (3-BP 1-IN-3SAT). For that purpose, we recall 3-BP 1-IN-3SAT as follows: Given a 3-CNF formula $\phi$ with $n$ positive variables $x_{1}, \ldots, x_{n}$ and $m$ clauses $C_{1}, \ldots, C_{m}$, such that each variable appears in at most three clauses, the goal is to check whether $\phi$ has a satisfying assignment such that every clause of $\phi$ has exactly one true literal [DF09].

Let each variable $x_{i}(1 \leq i \leq n)$ be represented by a variable gadget as shown in Figure $3.6(\mathrm{a})$, Let each clause $C_{j}(1 \leq j \leq m)$ be represented by a clause gadget as 
shown in Figure 3.6(b), If a variable $x_{i}$ is the 1 st, 2 nd, or 3 rd literal of a clause $C_{j}$, then the corresponding vertex labeled $x_{i}$ is connected to a sink labeled $C_{j}$ through a pair of vertices labeled $\left\{y_{j 1}, z_{j 1}\right\},\left\{y_{j 2}, z_{j 2}\right\}$, or $\left\{y_{j 3}, z_{j 3}\right\}$, respectively.

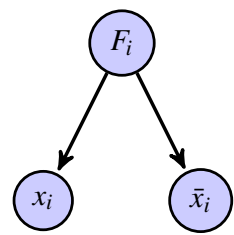

(a) Variable

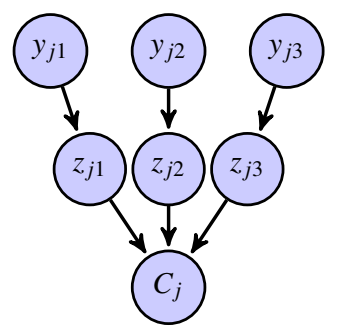

(b) Clause

Figure 3.6: Gadgets used to represent variables and clauses.
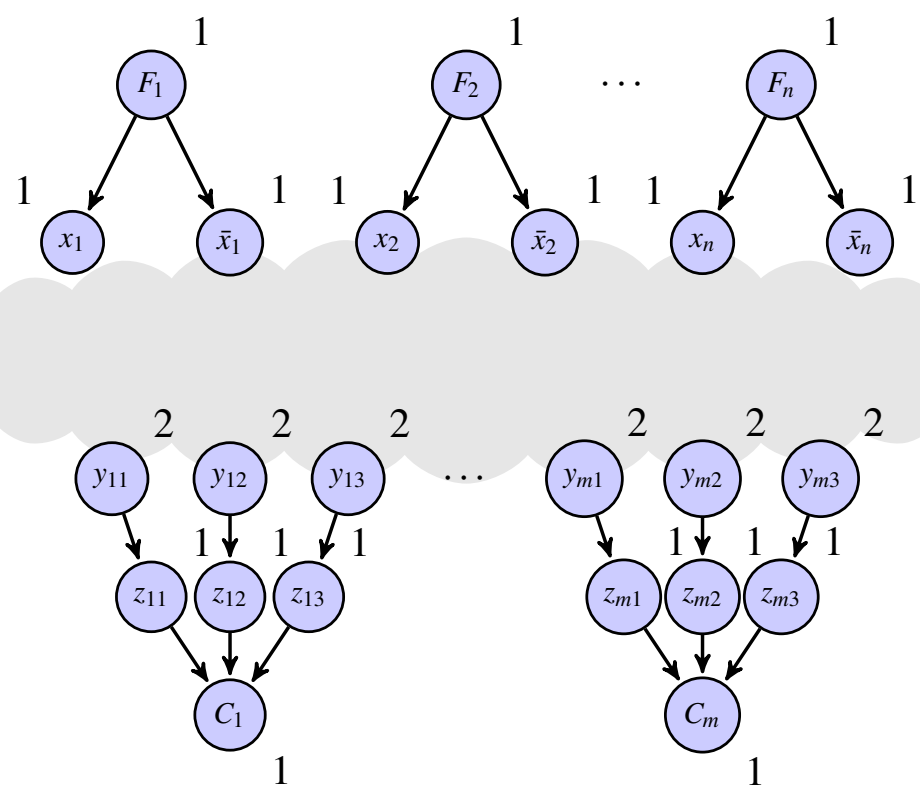

Figure 3.7: Reduction from 3-BP 1-IN-3SAT to $\mathrm{CN}_{d e c}\langle[2], 2,4\rangle$. The edges connecting variable gadgets to clause gadgets belong in the area with the shaded cloud.

We now construct an instance $I^{\prime}$ of $\mathrm{CN}_{d e c}\langle[2], 2,4\rangle$ as shown in Figure 3.7. The resulting DAG $G$ represents a combinatorial circuit. Let $U$ denote the set of all vertices labeled $x_{i}$ or $\bar{x}_{i}(1 \leq i \leq n)$. There are $n$ sources labeled $F_{i}(1 \leq i \leq n)$ and $m$ sinks labeled $C_{j}(1 \leq j \leq m)$. They are connected through some vertices in $U$ and $3 \cdot m$ pairs 
of vertices labeled $\left\{y_{j p}, z_{j p}\right\}(1 \leq j \leq m, 1 \leq p \leq 3)$. Each $y_{j p}$ is connected to exactly one variable gadget. Every $x_{i}, \bar{x}_{i} \in U$, every $F_{i}, z_{j p}$, and $C_{j}$ has a weight of 1 . Every $y_{j p}$ has a weight of 2 . We set $d=0$ and let $D$ be any positive integer. All vertices are given a delay of 0 . The cluster capacity $M$ is set to 2 , and we set $k=3 \cdot D$. The description of $I^{\prime}$ is complete.

Observe that $I^{\prime}$ can be constructed from $I$ in polynomial time. In order to complete the proof of the theorem, we show that $I$ is a "yes" instance of 3-BP 1-IN-3SAT if and only if $I^{\prime}$ is a "yes" instance of $\mathrm{CN}_{d e c}\langle[2], 2,4\rangle$.

Suppose that $I$ is a "yes" instance of 3-BP 1-IN-3SAT. This means that there exists an assignment of $\phi$ such that every clause has exactly one true literal. If a literal $x_{i}$ is set to true, then the corresponding vertex $x_{i}$ is clustered alone. However, if a literal $x_{i}$ is set to false, then the corresponding vertex is clustered with $F_{i}$. Since $M=2$, every $y_{j p}$ must be clustered alone. Since each clause $C_{j}$ has exactly one true literal, the vertex $z_{j p}$ along the path of the vertex $x_{i}$ corresponding to that true literal is clustered with $C_{j}$. The resulting delay-length of the corresponding source to sink path is $3 \cdot D$. The other two vertices belonging to the same clause gadget are clustered alone. Observe that the cluster capacity constraint is satisfied, and the maximum delay-length of any path from a source $F_{i}$ to a $\operatorname{sink} C_{j}$ is $3 \cdot D$. This means that $I^{\prime}$ is a "yes" instance of $\mathrm{CN}_{d e c}\langle[2], 2,4\rangle$.

Conversely, suppose that $I^{\prime}$ is a "yes" instance of $\mathrm{CN}_{d e c}\langle[2], 2,4\rangle$. This means that there is a way of partitioning the vertices of $G$ into clusters of capacity $M=2$, and the maximum delay-length of any path from a source to a sink is at most $3 \cdot D$.

Since $M=2$, again notice that every $y_{j p}$ must be clustered alone. Each sink $C_{j}$ is clustered with at most one vertex, so the remaining two $z_{j p}$ vertices are clustered alone. Consider a vertex $x_{i}$ along a path corresponding to a $z_{j p}$ that is clustered alone. Since such a source to sink path has a delay-length of at least $3 \cdot D$, then the source $F_{i}$ must be clustered with vertex $x_{i}$. Otherwise, the delay-length of the path would be $4 \cdot D>3 \cdot D=k$. Furthermore, since the cluster capacity is satisfied, $F_{i}$ can be clustered 
with either $x_{i}$ or $\bar{x}_{i}$ (but not both). Take each literal that corresponds to a vertex $x_{i}$ clustered with $F_{i}$ and set its value to false. Take each literal $x_{i}$ that corresponds to a vertex $x_{i}$ clustered alone and set its value to true. Notice that any $z_{j p}$ along a path in which vertex $x_{i}$ is clustered alone must be clustered with the $\operatorname{sink} C_{j}$. Otherwise, the delay-length of the path would be $4 \cdot D>3 \cdot D=k$. Observe that exactly one true literal appears in every clause. Thus, a satisfying clustering for $G$ yields a satisfying assignment for $\phi$. Hence, $I$ is a "yes" instance of 3-BP 1-IN-3SAT.

The proof of Theorem 3.1.4 implies an inapproximability result for $\mathrm{CN}\langle[2], 2,4\rangle$.

Corollary 3.1.4. $C N\langle[2], 2,4\rangle$ does not admit a $\left(\frac{4}{3}-\varepsilon\right)$-approximation algorithm for each $\varepsilon>0$, unless $\mathbf{P}=\mathbf{N P}$.

Proof. By way of contradiction, suppose there exists a $\left(\frac{4}{3}-\varepsilon\right)$-approximation algorithm for $\mathrm{CN}_{d e c}\langle[2], 2,4\rangle$. We construct a polynomial time algorithm for 3-BP 1-IN3SAT as follows:

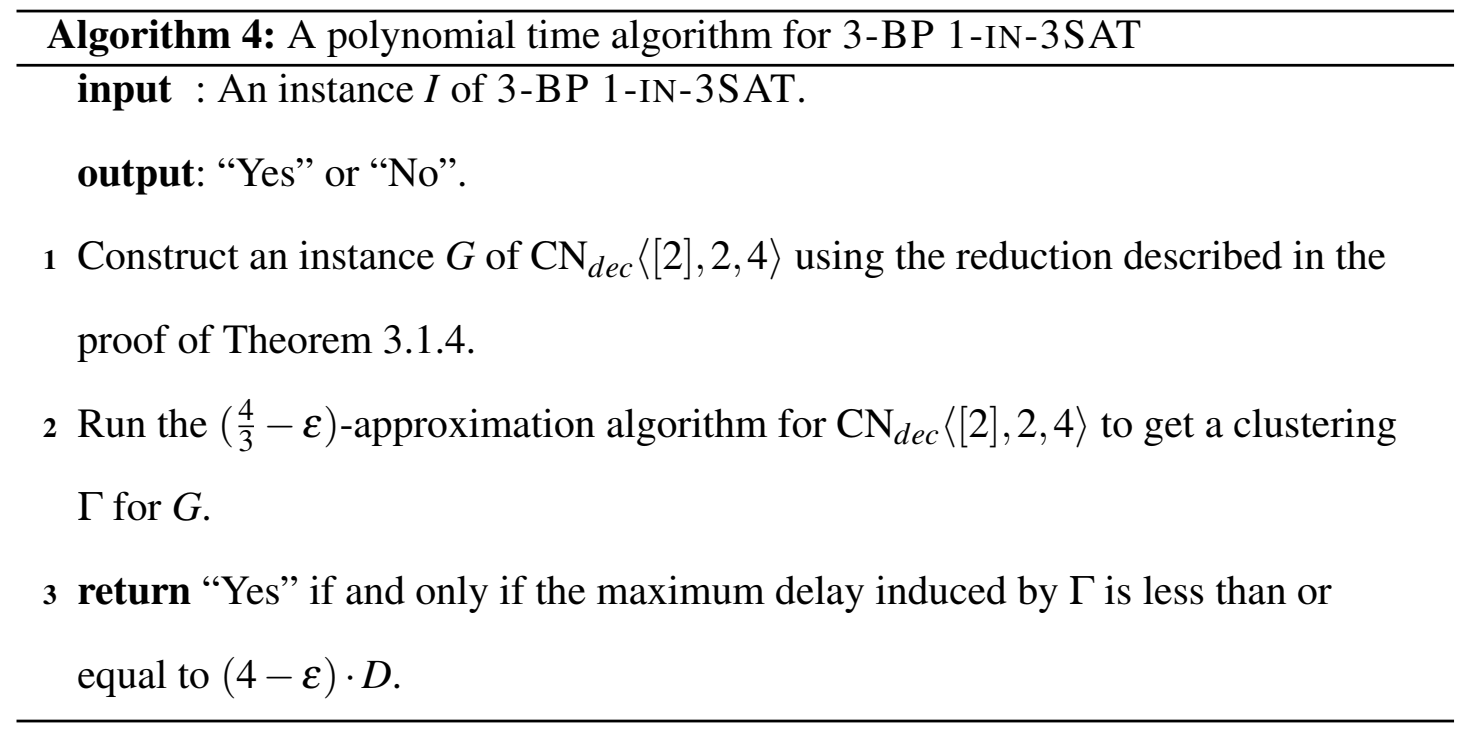

Let $O P T$ denote the delay of the optimal clustering of $G$. If $I$ is a "yes" instance of 3-BP 1 -IN-3SAT, then $O P T \leq 3 \cdot D$. Moreover, the maximum delay of any clustering solution of $G$ returned by the $\left(\frac{4}{3}-\varepsilon\right)$-approximation algorithm for $\mathrm{CN}_{d e c}\langle[2], 2,4\rangle$ is at 
most $(4-\varepsilon) \cdot D$. Otherwise, the maximum delay of any clustering solution of $G$ must be at least $4 \cdot D$. Thus, the $\left(\frac{4}{3}-\varepsilon\right)$-approximation algorithm solves the instance $I$ of 3-BP 1-IN-3SAT exactly.

The next theorem implies NP-hardness of $\mathrm{CN}\langle[2], 2,4\rangle-$ a restriction of $\mathrm{CN}\langle W, 2,3\rangle$.

Theorem 3.1.5. $C N_{d e c}\langle[2], 2,3\rangle$ is NP-complete.

Proof. It is clear that $\mathrm{CN}_{d e c}\langle[2], 2,3\rangle$ is in NP. This follows from the well-known fact that a maximum weighted path in an edge-weighted DAG can be found in polynomial time [CLRS09].

In order to establish NP-hardness of $\mathrm{CN}_{d e c}\langle[2], 2,3\rangle$, we reduce from 1-IN-3SAT and recall the problem as follows:

1-IN-3SAT: Given a 3-CNF formula $\phi$ with $n$ variables $x_{1}, \ldots, x_{n}$ and $m$ clauses $C_{1}, \ldots, C_{m}$, the goal is to check whether $\phi$ has a satisfying assignment such that every clause of $\phi$ has exactly one true literal. Without loss of generality, for all $i \in\{1, \ldots, n\}$ we assume that each variable $x_{i}$ in $\phi$ appears at most 3 times and each literal at most twice. (Any 3SAT instance can be transformed to satisfy these properties in polynomial time [Pap94].)

Let each variable $x_{i}(1 \leq i \leq n)$, be represented by a variable gadget as shown in Figure 3.8(a) Let each clause $C_{j}(1 \leq j \leq m)$, be represented by a clause gadget as shown in Figure 3.8(b). If a variable $x_{i}$ or its complement $\bar{x}_{i}$ is the 1st, 2nd, or 3rd literal of a clause $C_{j}$, then the corresponding vertex labeled $x_{i}\left(\right.$ or $\left.\bar{x}_{i}\right)$ is connected to a sink labeled $C_{j}$ by traversing the edge $\left(y_{j 1}, z_{j 1}\right),\left(y_{j 2}, z_{j 2}\right)$, or $\left(y_{j 3}, z_{j 3}\right)$, respectively.

We now construct an instance $I^{\prime}$ of $\mathrm{CN}_{d e c}\langle[2], 2,3\rangle$ as shown in Figure 3.9. The resulting DAG $G$ represents a combinatorial circuit. Let $U$ be the set of all vertices labeled $x_{i}$ or $\bar{x}_{i}(1 \leq i \leq n)$. There are $n$ sources $S_{i}$ connected to $m \operatorname{sinks} C_{j}(1 \leq j \leq m)$ 


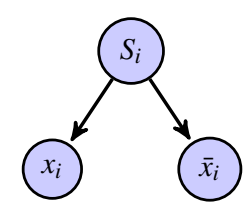

(a) Variable

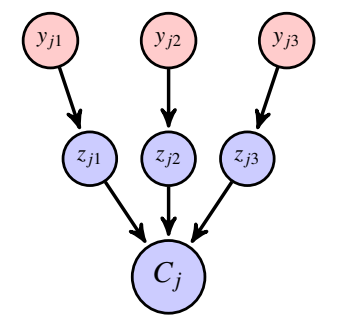

(b) Clause

Figure 3.8: Gadgets used to represent variables and clauses.

through some vertices in $U$ and $3 \cdot m$ pairs of vertices labeled $y_{j p}$ and $z_{j p}(1 \leq j \leq$ $m, 1 \leq p \leq 3)$. Notice that every $y_{j p}$ is connected to exactly one vertex gadget. For each $i$, we have that $x_{i}$ and $\bar{x}_{i}$ cannot both be connected to the same clause gadget. We let every vertex in $U$, and each $S_{i}, z_{j p}$, and $C_{j}$ have a weight of 1 . We let each $y_{j p}$ have a weight of 2 . We set $d=0$ and let $D$ be any positive integer. All vertices are given a delay of 0 . The cluster capacity $M$ is set to 2 , and we let $k=3 \cdot D$. The description of $I^{\prime}$ is complete.
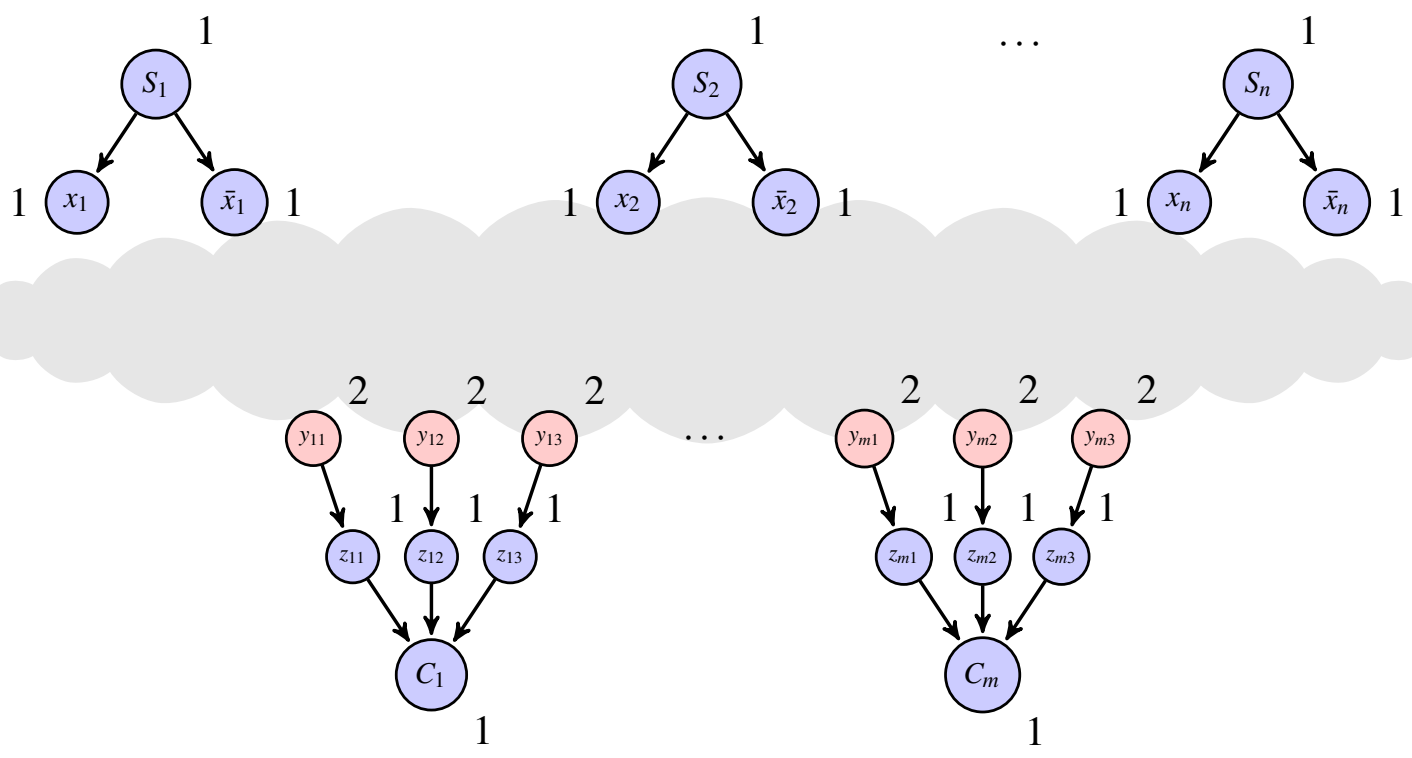

Figure 3.9: Reduction from 1-IN-3SAT to $\mathrm{CN}_{d e c}\langle[2], 2,3\rangle$. The edges connecting variable gadgets to clause gadgets belong in the shaded area.

Observe that $I^{\prime}$ can be constructed from $I$ in polynomial time. In order to complete 
the proof of the theorem, we show that $I$ is a "yes" instance of 1-IN-3SAT, if and only if $I^{\prime}$ is a "yes" instance of $\mathrm{CN}_{d e c}\langle[2], 2,3\rangle$.

Suppose that $I$ is a "yes" instance of 1 -IN-3SAT. This means that there exists an assignment of $\phi$ such that every clause has exactly one true literal. If a literal is set to true, then the corresponding vertex $\left(x_{i}\right.$ or $\left.\bar{x}_{i}\right)$ should be clustered alone. But, if a literal is set to false, then the corresponding vertex should be clustered with $S_{i}$. Since $M=2$, every $y_{j p}$ must be clustered alone. Each clause $C_{j}$ has exactly one true literal, so the vertex $z_{j p}$ along the path of the vertex $\left(x_{i}\right.$ or $\left.\bar{x}_{i}\right)$ corresponding to that literal should be clustered with the sink $C_{j}$. Every $z_{j p}$ not clustered with $C_{j}$ must be clustered alone. Observe that the cluster capacity constraint is met, and the maximum delay-length of any path from a source $S_{i}$ to a $\operatorname{sink} C_{j}$ is $3 \cdot D$. This means that $I^{\prime}$ is a "yes" instance of $\mathrm{CN}_{\text {dec }}\langle[2], 2,3\rangle$.

Conversely, suppose that $I^{\prime}$ is a "yes" instance of $\mathrm{CN}_{d e c}\langle[2], 2,3\rangle$. This means that there is a way of packing the vertices of $G$ into clusters of capacity $M=2$, such that the delay-length of any path from a source to a sink is at most $3 \cdot D$.

Since $M=2$, again notice that every $y_{j p}$ must be clustered alone. Each sink $C_{j}$ is clustered with at most one $z_{j p}$. So, the remaining two $z_{j p}$ vertices are clustered alone. Consider a vertex $x_{i}$ (or $\bar{x}_{i}$ ) along a path corresponding to a $z_{j p}$ that is clustered alone. Since such a source to sink path has delay-length at least $3 \cdot D$, then the source $S_{i}$ must be clustered with vertex $x_{i}$ (or $\bar{x}_{i}$ ). Otherwise, the delay-length of the path would be $4 \cdot D>3 \cdot D=k$. Furthermore, since the cluster capacity constraint is satisfied, $S_{i}$ can be clustered with either $x_{i}$ or $\bar{x}_{i}$ (but not both). Take each literal that corresponds to a vertex $x_{i}$ (or $\bar{x}_{i}$ ) that is clustered with $S_{i}$ and set its value to false. Take each literal that corresponds to a vertex $x_{i}$ (or $\bar{x}_{i}$ ) that is clustered alone and set its value to true. Finally, notice that any $z_{j p}$ along a path in which vertex $x_{i}$ (or $\bar{x}_{i}$ ) is clustered alone must be clustered with the $\operatorname{sink} C_{j}$. Otherwise, the delay-length of the path would be $4 \cdot D>3 \cdot D=k$. Observe that exactly one true literal appears in every clause. Thus, 
a satisfying clustering for $G$ yields a satisfying assignment for $\phi$. Hence, $I$ is a "yes" instance of 1-IN-3SAT.

The proof of Theorem 3.1 .5 implies an inapproximability result for $\mathrm{CN}\langle[2], 2,3\rangle$.

Corollary 3.1.5. $\mathrm{CN}\langle[2], 2,3\rangle$ does not admit a $\left(\frac{4}{3}-\varepsilon\right)$-approximation algorithm for each $\varepsilon>0$, unless $\mathbf{P}=\mathbf{N P}$.

Proof. By way of contradiction, suppose there exists a $\left(\frac{4}{3}-\varepsilon\right)$-approximation algorithm for $\mathrm{CN}_{d e c}\langle[2], 2,3\rangle$. We construct a polynomial time algorithm for 1-IN-3SAT as follows:

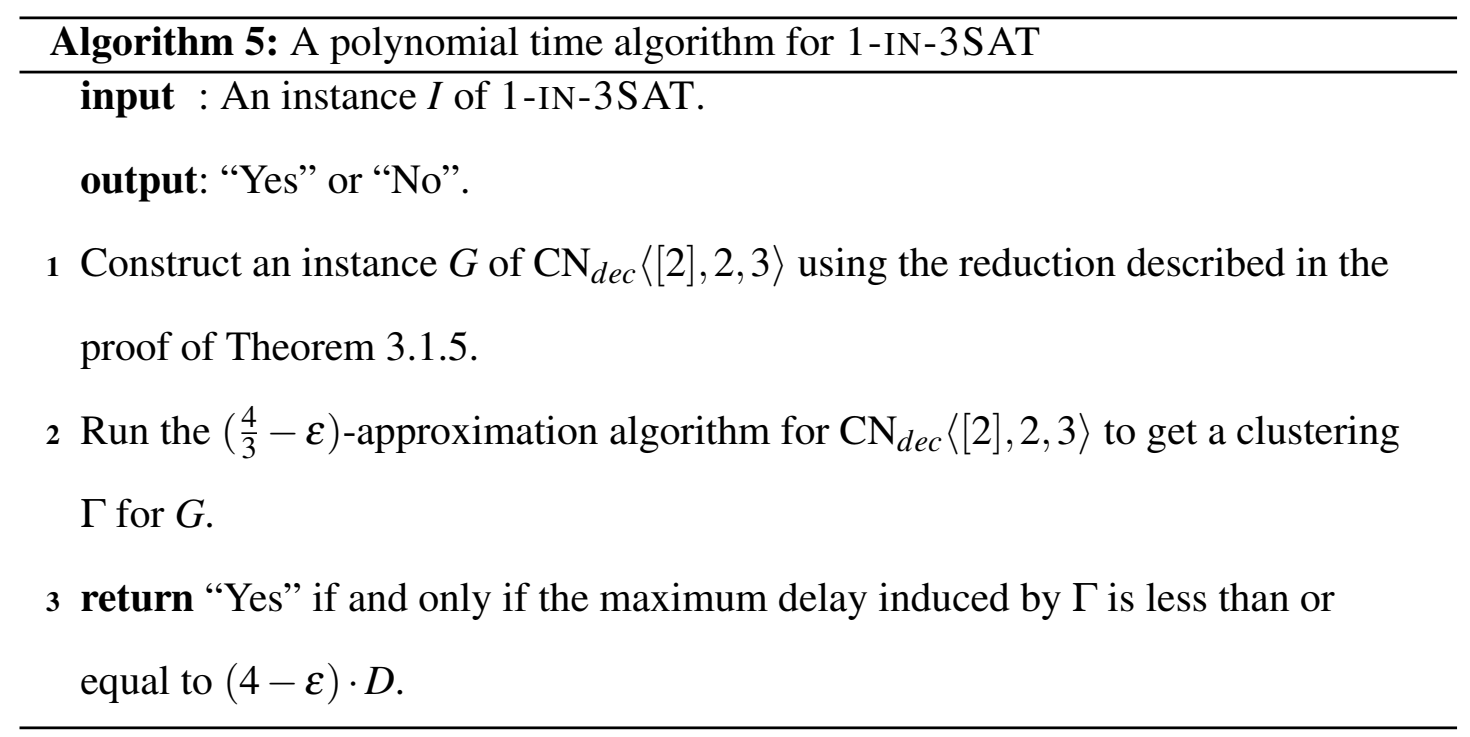

Let $O P T$ denote the delay of the optimal clustering of $G$. If $I$ is a "yes" instance of 1 -IN-3SAT, then $O P T \leq 3 \cdot D$. Moreover, the maximum delay of any clustering solution of $G$ returned by the $\left(\frac{4}{3}-\varepsilon\right)$-approximation algorithm for $\mathrm{CN}_{d e c}\langle[2], 2,3\rangle$ is at most $(4-\varepsilon) \cdot D$. Otherwise, the maximum delay of any clustering solution of $G$ must be at least $4 \cdot D$. Thus, the $\left(\frac{4}{3}-\varepsilon\right)$-approximation algorithm solves the instance $I$ of $1-$ IN-3SAT exactly. 
In [Kag03], $\mathrm{CN}$ is considered under area constraints and pin constraints, separately. The decision version of the area-constrained problem is formulated as follows: Given a directed acyclic graph $G(V, E)$ representing a combinatorial circuit, a delay $\delta(v)$ and area $\alpha(v)$ for each $v \in V$, an inter-cluster delay constant $D \geq 0$, a cluster area bound $M$, and a maximum delay bound $B$, determine whether there exists a clustering with no replication so that in each cluster $C, \sum_{v \in C} \alpha(v) \leq M$, and for any path $P=\left(p_{1}, p_{2}, \ldots, p_{n}\right)$ from a primary input to a primary output, $\sum_{i=1}^{n} \delta\left(p_{i}\right)+k \cdot D \leq B$, where $k=\mid\left\{\left(p_{i}, p_{i+1}\right):\left(p_{i}, p_{i+1} \in P\right) \wedge\left(p_{i}, p_{i+1}\right.\right.$ appear in different clusters $\left.)\right\} \mid$. The decision version of the pin-constrained problem has an analogous formulation. However, the area of each cluster $C$ is not restricted, while the total number of I/O pins of each cluster must not exceed a given constant $Q$.

The next results establish NP-hardness and inapproximability of $\mathrm{CN}\langle[4], 5, \Delta\rangle$.

Theorem 3.1.6. $C N\langle[4], 5, \Delta\rangle$ is NP-hard.

Proof. We recall $\mathrm{CN}\langle[4], 5, \Delta\rangle$ as follows: Given a DAG $G=(V, E)$, with vertex-weight function $w: V \rightarrow\{1,2,3,4\}, \delta(v)=0 \forall v \in V$, maximum degree $\Delta$, constants $d$ and $D$, and cluster capacity $M=5$, the goal is to partition $V$ into clusters such that the weight of each cluster is bounded by $M$, and the maximum delay-length of any path from a source to a sink of $G$ is minimized.

To show that $\mathrm{CN}\langle[4], 5, \Delta\rangle$ is NP-hard, we reduce from 3SAT (cf. Theorem 2.1 in [Kag03]). For that purpose, we recall 3SAT as follows: Given a 3-CNF formula $\phi$ with $n$ variables $x_{1}, \ldots, x_{n}$ and $m$ clauses $C_{1}, \ldots, C_{m}$, the goal is to check whether $\phi$ has a satisfying assignment.

Let each variable $x_{i}(1 \leq i \leq n)$ be represented by a variable gadget as shown in Figure $3.10(\mathrm{a})$. Let each clause $C_{j}(1 \leq j \leq m)$ be represented by a clause gadget as shown in Figure 3.10(b), If a variable $x_{i}$ or its complement $\bar{x}_{i}$ is the $p$ th literal of a clause $C_{j}$, where $p \in\{1,2,3\}$, then we add edges $\left(x_{i}, z_{j p}\right)$ or $\left(\bar{x}_{i}, z_{j p}\right)$, respectively. The resulting DAG $G$ represents a combinatorial circuit. Let $U$ denote the set of all 


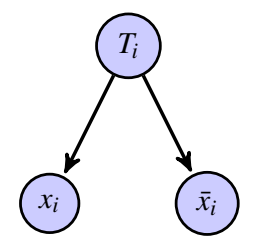

(a) Variable

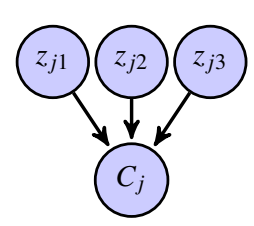

(b) Clause

Figure 3.10: Gadgets used to represent variables and clauses.

vertices labeled $x_{i}$ or $\bar{x}_{i}(1 \leq i \leq n)$. There are $n$ sources labeled $T_{i}(1 \leq i \leq n)$ and $m$ sinks labeled $C_{j}(1 \leq j \leq m)$. They are connected through some vertices in $U$ and $3 \cdot m$ vertices labeled $z_{j p}(1 \leq j \leq m, 1 \leq p \leq 3)$. Each $z_{j p}$ is connected to exactly one variable gadget. For every $j$, no two vertices in the set $\left\{z_{j 1}, z_{j 2}, z_{j 3}\right\}$ are adjacent to both $x_{i}$ and $\bar{x}_{i}$ of the same variable gadget. In other words, $x_{i}$ and $\bar{x}_{i}$ cannot both be connected to the same clause gadget. Every $T_{i}$ and $C_{j}$ has a weight of 1 , every $x_{i}, \bar{x}_{i} \in U$ has a weight of 4 , and every $z_{j p}$ has a weight of 2 . Let $d=0$ and let $D$ be any positive integer. All vertices are given a delay of 0 . The cluster capacity $M$ is set to 5 , and set $k=2 \cdot D$. It is shown that an instance $I$ of 3 SAT is a "yes" instance if and only if an instance $I^{\prime}$ of $\mathrm{CN}\langle[4], 5, \Delta\rangle$ is a "yes" instance.

Corollary 3.1.6. $C N\langle[4], 5, \Delta\rangle$ does not admit a $\left(\frac{3}{2}-\varepsilon\right)$-approximation algorithm for any $\varepsilon>0$, unless $\mathbf{P}=\mathbf{N P}$.

Proof. By way of contradiction, suppose there exists a $\left(\frac{3}{2}-\varepsilon\right)$-approximation algorithm for $\mathrm{CN}\langle[4], 5, \Delta\rangle$. We construct a polynomial time algorithm for 3SAT as follows: 


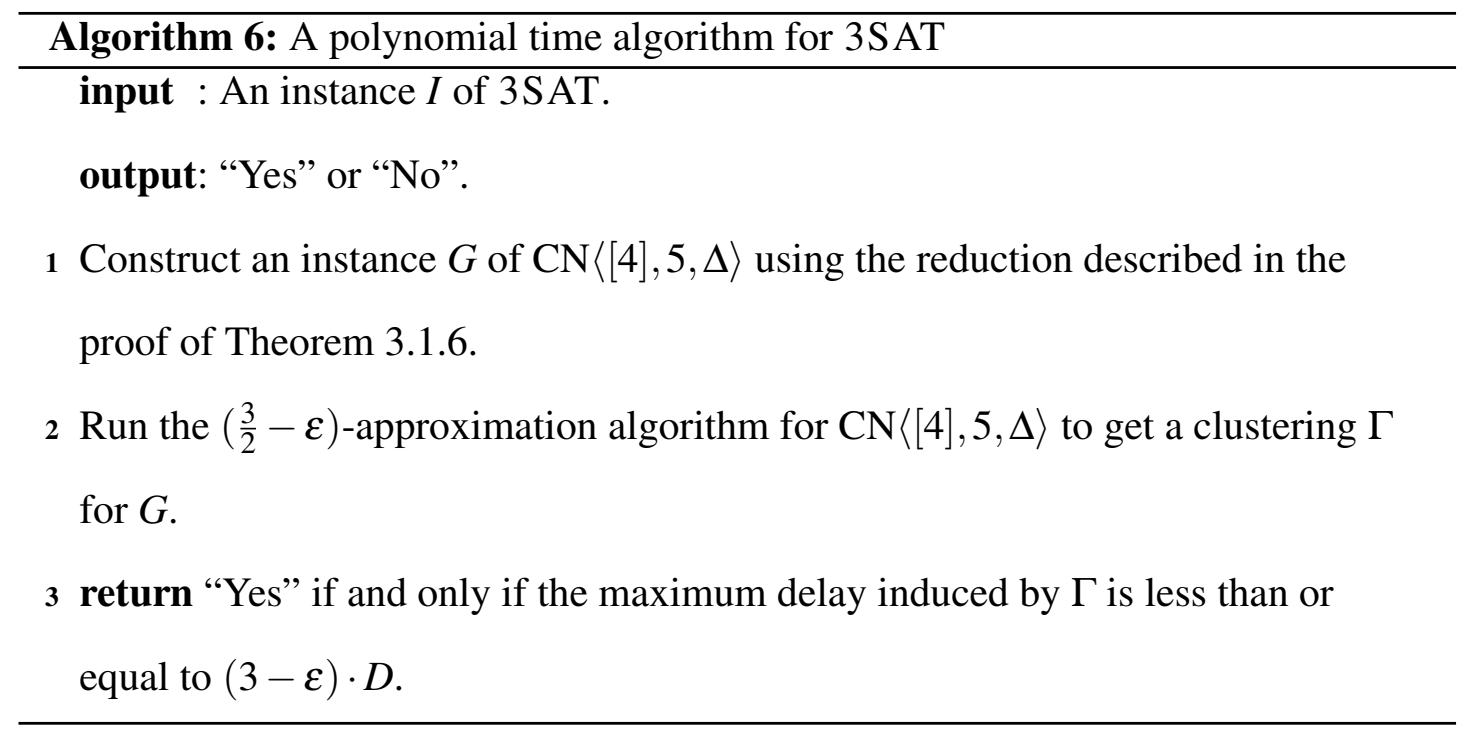

Let $O P T$ denote the delay of the optimal clustering of $G$. If $I$ is a "yes" instance of 3SAT, then $O P T \leq 2 \cdot D$. Moreover, the maximum delay of any clustering solution of $G$ returned by the $\left(\frac{3}{2}-\varepsilon\right)$-approximation algorithm for $\mathrm{CN}\langle[4], 5, \Delta\rangle$ is at most $(3-\varepsilon) \cdot D$. Otherwise, the maximum delay of any clustering solution of $G$ must be at least $3 \cdot D$. Thus, the $\left(\frac{3}{2}-\varepsilon\right)$-approximation algorithm solves the instance $I$ of 3SAT exactly.

We further observe that if we reduce from the variant of 3SAT such that each variable occurs at most three times and each literal occurs at most twice in the Boolean formula, then we obtain the next result.

Theorem 3.1.7. $C N\langle[4], 5,3\rangle$ is NP-hard.

Proof. We recall $\mathrm{CN}\langle[4], 5,3\rangle$ as follows: Given a DAG $G=(V, E)$, with vertex-weight function $w: V \rightarrow\{1,2,3,4\}, \delta(v)=0 \forall v \in V$, maximum degree $\Delta=3$, constants $d$ and $D$, and cluster capacity $M=5$, the goal is to partition $V$ into clusters such that the weight of each cluster is bounded by $M$, and the maximum delay-length of any path from a source to a sink of $G$ is minimized.

To show that $\mathrm{CN}\langle[4], 5,3\rangle$ is NP-hard, we reduce (in polynomial time) from a variant of 3SAT (cf. Theorem 2.1 in [ $[\overline{K a g 03}]$ ). For that purpose, we recall the variant of 
3SAT as follows:

$3 \mathrm{SAT}_{\leq 3, \leq 2}$ : Given a 3 -CNF formula $\phi$ with $n$ variables $x_{1}, \ldots, x_{n}$ and $m$ clauses $C_{1}, \ldots, C_{m}$, such that each variable occurs at most three times and each literal occurs at most twice, the goal is to check whether $\phi$ has a satisfying assignment. Note that the requirement that each clause has exactly three literals is relaxed in this restriction of 3SAT. Any variable, say $x_{i}$, with $q$ occurrences (for some $q>3$ ) can be replaced with $q$ new variables $w_{1}, \ldots, w_{q}$. The clauses $\left(\bar{w}_{1} \vee w_{2}\right) \wedge\left(\bar{w}_{2} \vee w_{3}\right) \wedge\left(\bar{w}_{q} \vee w_{1}\right)$ can then be added to $\phi$ to ensure that the $q$ new variables retain the truth assignment of the original variable $x_{i}[$ Pap94].

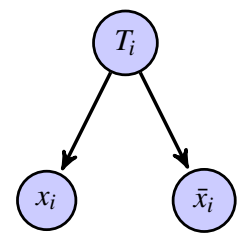

(a) Variable

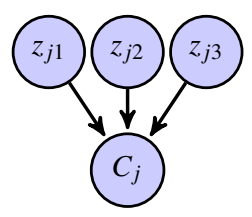

(b) Clause

Figure 3.11: Gadgets used to represent variables and clauses.

Given an instance $I$ of $3 \mathrm{SAT}_{\leq 3, \leq 2}$, we construct an instance $I^{\prime}$ of $\mathrm{CN}\langle[4], 5,3\rangle$. Let each variable $x_{i}(1 \leq i \leq n)$ be represented by a variable gadget as shown in Figure 3.11(a), Let each clause $C_{j}(1 \leq j \leq m)$ be represented by a clause gadget as shown in Figure 3.11(b), If a variable $x_{i}$ or its complement $\bar{x}_{i}$ is the $p$ th literal of a clause $C_{j}$, where $p \in\{1,2,3\}$, then we add edges $\left(x_{i}, z_{j p}\right)$ or $\left(\bar{x}_{i}, z_{j p}\right)$, respectively. The resulting DAG $G$ represents a combinatorial circuit. Let $U$ denote the set of all vertices labeled $x_{i}$ or $\bar{x}_{i}(1 \leq i \leq n)$. There are $n$ sources labeled $T_{i}(1 \leq i \leq n)$ and $m$ sinks labeled $C_{j}$ $(1 \leq j \leq m)$. They are connected through some vertices in $U$ and $3 \cdot m$ vertices labeled $z_{j p}(1 \leq j \leq m, 1 \leq p \leq 3)$. Each $z_{j p}$ is connected to exactly one variable gadget. For every $j$, no two vertices in the set $\left\{z_{j 1}, z_{j 2}, z_{j 3}\right\}$ are adjacent to both $x_{i}$ and $\bar{x}_{i}$ of the same variable gadget. In other words, $x_{i}$ and $\bar{x}_{i}$ cannot both be connected to the same clause gadget. Every $T_{i}$ and $C_{j}$ has a weight of 1 , every $x_{i}, \bar{x}_{i} \in U$ has a weight of 4 , and 
every $z_{j p}$ has a weight of 2 . We set $d=0$ and let $D$ be any positive integer. All vertices are given a delay of 0 . The cluster capacity $M$ is set to 5 , and we set $k=2 \cdot D$. The description of $I^{\prime}$ is complete.

Observe that an instance $I^{\prime}$ of $\mathrm{CN}\langle[4], 5,3\rangle$, constructed from any instance $I$ of $3 \mathrm{SAT}_{\leq 3, \leq 2}$, has maximum degree three. We can show (as in $[\operatorname{Kag} 03]$ ), that $I$ is a "yes" instance of $3 \mathrm{SAT}_{\leq 3, \leq 2}$ if and only if $I^{\prime}$ is a "yes" instance of $\mathrm{CN}\langle[4], 5,3\rangle$, as desired.

Corollary 3.1.7. $C N\langle[4], 5,3\rangle$ does not admit a $\left(\frac{3}{2}-\varepsilon\right)$-approximation algorithm for any $\varepsilon>0$, unless $\mathbf{P}=\mathbf{N P}$.

Proof. By way of contradiction, suppose there exists a $\left(\frac{3}{2}-\varepsilon\right)$-approximation algorithm for $\mathrm{CN}\langle[4], 5,3\rangle$. We construct a polynomial time algorithm for $3 \mathrm{SAT}_{\leq 3, \leq 2}$ as follows:

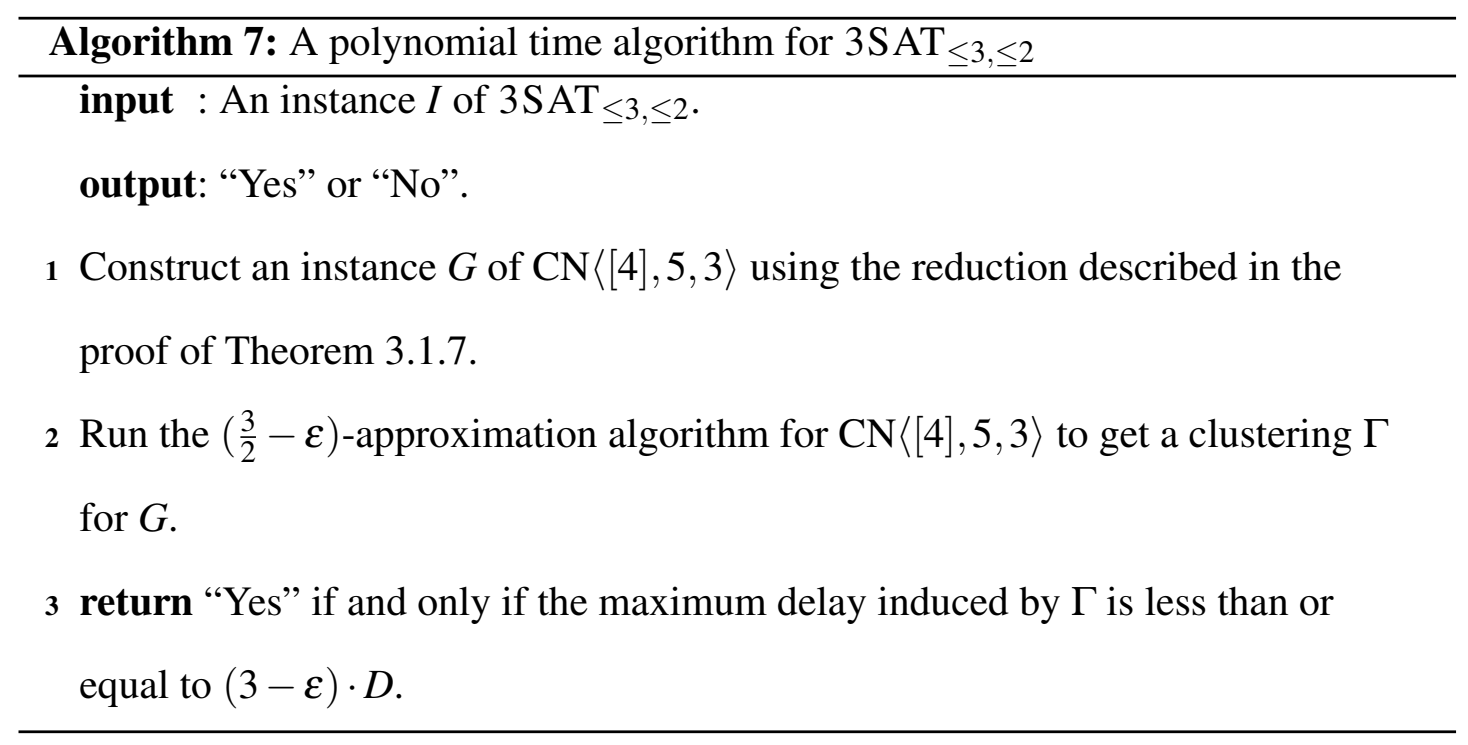

Let $O P T$ denote the delay of the optimal clustering of $G$. If $I$ is a "yes" instance of $3 \mathrm{SAT}_{\leq 3, \leq 2}$, then $O P T \leq 2 \cdot D$. Moreover, the maximum delay of any clustering solution of $G$ returned by the $\left(\frac{3}{2}-\varepsilon\right)$-approximation algorithm for $\mathrm{CN}\langle[4], 5,3\rangle$ is at most $(3-\varepsilon) \cdot D$. Otherwise, the maximum delay of any clustering solution of $G$ must be at least $3 \cdot D$. Thus, the $\left(\frac{3}{2}-\varepsilon\right)$-approximation algorithm solves the instance $I$ of $3 \mathrm{SAT}_{\leq 3, \leq 2}$ exactly. 
In [AFIM06], $\operatorname{MBT}(B)$ is shown to be NP-hard. They formulate $\operatorname{MBT}(B)$ as follows: Given a DAG $G=(V, A)$ and a block size $B$, the goal is to find a packing $\mathscr{P}$ that minimizes the block transfer $b t_{\mathscr{P}}(G)$ such that the size of every block $P_{i} \in \mathscr{P}$ is bounded by $B$. Furthermore, they obtain a $\left(\frac{3}{2}-\varepsilon\right)$-inapproximability bound and a $(2-\varepsilon)$-approximability bound for $\operatorname{MBT}(2)$, where $\varepsilon=\frac{2}{h}$ when height $h \geq 4$ is even and $\varepsilon=\frac{2}{h+1}$ when $h \geq 3$ is odd.

The next results establish NP-hardness and inapproximability of $\mathrm{CN}\langle N, 2,5\rangle$.

Theorem 3.1.8. $C N\langle N, 2,5\rangle$ is NP-hard.

Proof. We recall $\mathrm{CN}\langle N, 2,5\rangle$ as follows: Given a DAG $G=(V, E)$, with $w(v)=1 \forall v \in$ $V, \delta(v)=0 \forall v \in V$, maximum degree $\Delta=5$, constants $d$ and $D$, and cluster capacity $M=2$, the goal is to partition $V$ into clusters such that the weight of each cluster is bounded by $M$, and the maximum delay-length of any path from a source to a sink of $G$ is minimized.

To show that $\mathrm{CN}\langle N, 2,5\rangle$ is NP-hard, we construct an instance $I^{\prime}$ of $\mathrm{CN}\langle N, 2,5\rangle$ from an instance $I$ of 3SAT as described in the proof of Theorem 5 in [AFIM06]. The theorem follows from the fact that $I^{\prime}$ is a "yes" instance if and only if $I$ is a "yes" instance.

Corollary 3.1.8. $C N\langle N, 2,5\rangle$ does not admit a $\left(\frac{3}{2}-\varepsilon\right)$-approximation algorithm for each $\varepsilon>0$, unless $\mathbf{P}=\mathbf{N P}$.

Proof. By way of contradiction, suppose there exists a $\left(\frac{3}{2}-\varepsilon\right)$-approximation algorithm for $\mathrm{CN}\langle N, 2,5\rangle$. We construct a polynomial time algorithm for 3SAT as follows: 


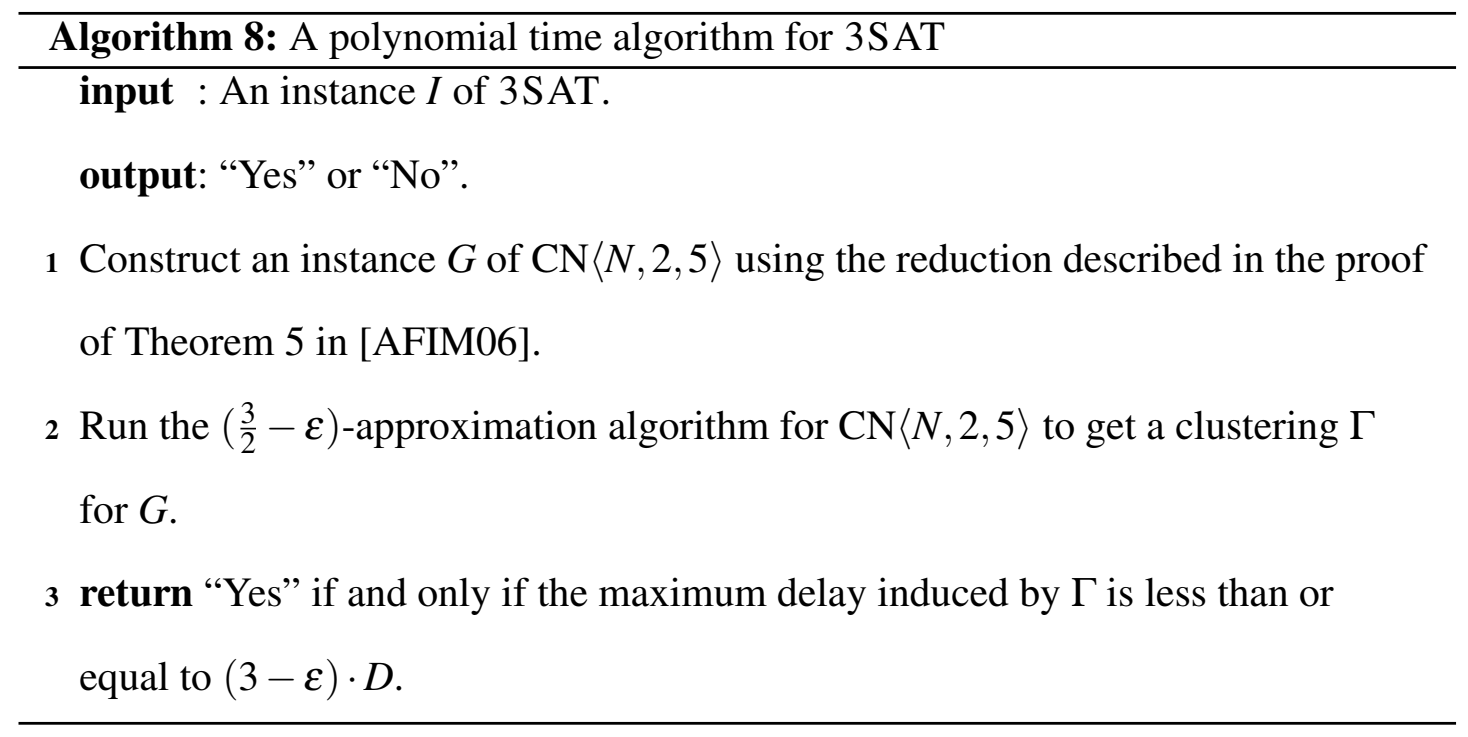

Let $O P T$ denote the delay of the optimal clustering of $G$. If $I$ is a "yes" instance of 3SAT, then $O P T \leq 2 \cdot D$. Moreover, the maximum delay of any clustering solution of $G$ returned by the $\left(\frac{3}{2}-\varepsilon\right)$-approximation algorithm for $\mathrm{CN}\langle N, 2,5\rangle$ is at most $(3-\varepsilon) \cdot D$. Otherwise, the maximum delay of any clustering solution of $G$ must be at least $3 \cdot D$. Thus, the $\left(\frac{3}{2}-\varepsilon\right)$-approximation algorithm solves the instance $I$ of 3SAT exactly.

We further observe that if we reduce from the variant of 3SAT such that each variable occurs at most three times and each literal occurs at most twice, then we obtain the next result.

Theorem 3.1.9. $C N\langle N, 2,4\rangle$ is NP-hard.

Proof. We recall $\mathrm{CN}\langle N, 2,4\rangle$ as follows: Given a DAG $G=(V, E)$, with $w(v)=1 \forall v \in$ $V, \delta(v)=0 \forall v \in V$, maximum degree $\Delta=4$, constants $d$ and $D$, and cluster capacity $M=2$, the goal is to partition $V$ into clusters such that the weight of each cluster is bounded by $M$, and the maximum delay-length of any path from a source to a sink of $G$ is minimized.

Consider the proof of Theorem 5 in [AFIM06]. To show that $\mathrm{CN}\langle N, 2,4\rangle$ is NPhard, we reduce (in polynomial time) from a variant of 3SAT, instead. For that purpose, 
we recall the variant of 3SAT as follows:

$3 \mathrm{SAT}_{\leq 3, \leq 2}$ : Given a 3 -CNF formula $\phi$ with $n$ variables $u_{1}, \ldots, u_{n}$ and $m$ clauses $C_{1}, \ldots, C_{m}$, such that each variable occurs at most three times and each literal occurs at most twice, the goal is to check whether $\phi$ has a satisfying assignment. Note that the requirement that each clause has exactly three literals is relaxed in this restriction of 3SAT. Any variable, say $u_{i}$, with $q$ occurrences (for some $q>3$ ) can be replaced with $q$ new variables $w_{1}, \ldots, w_{q}$. The clauses $\left(\bar{w}_{1} \vee w_{2}\right) \wedge\left(\bar{w}_{2} \vee w_{3}\right) \wedge\left(\bar{w}_{q} \vee w_{1}\right)$ can then be added to $\phi$ to ensure that the $q$ new variables retain the truth assignment of the original variable $u_{i}[$ Pap94].

Given an instance $I$ of $3 \mathrm{SAT}_{\leq 3, \leq 2}$, we construct an instance $I^{\prime}$ of $\mathrm{CN}\langle N, 2,4\rangle$ using the reduction described in the proof of Theorem 5 in [AFIM06]. Notice that every instance $I^{\prime}$ of $\mathrm{CN}\langle N, 2,4\rangle$ has maximum degree four. It is easy to see based on the proof in [AFIM06], that $I$ is a "yes" instance of $3 \mathrm{SAT}_{\leq 3, \leq 2}$ if and only if $I^{\prime}$ is a "yes" instance of $\mathrm{CN}\langle N, 2,4\rangle$, as desired.

Corollary 3.1.9. $C N\langle N, 2,4\rangle$ does not admit a $\left(\frac{3}{2}-\varepsilon\right)$-approximation algorithm for each $\varepsilon>0$, unless $\mathbf{P}=\mathbf{N P}$.

Proof. By way of contradiction, suppose there exists a $\left(\frac{3}{2}-\varepsilon\right)$-approximation algorithm for $\mathrm{CN}\langle N, 2,4\rangle$. We construct a polynomial time algorithm for $3 \mathrm{SAT}_{\leq 3, \leq 2}$ as follows: 


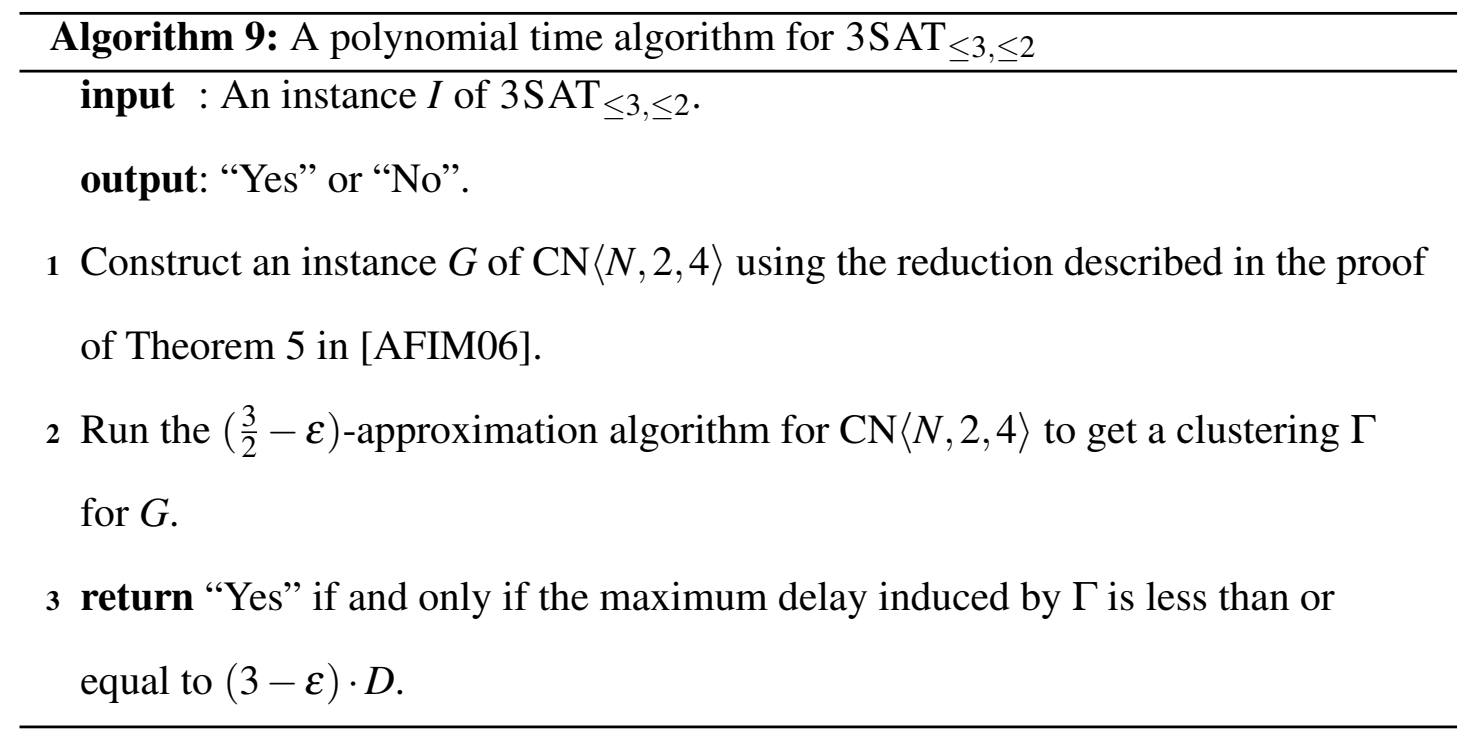

Let $O P T$ denote the delay of the optimal clustering of $G$. If $I$ is a "yes" instance of $3 \mathrm{SAT}_{\leq 3, \leq 2}$, then $O P T \leq 2 \cdot D$. Moreover, the maximum delay of any clustering solution of $G$ returned by the $\left(\frac{3}{2}-\varepsilon\right)$-approximation algorithm for $\mathrm{CN}\langle N, 2,4\rangle$ is at most $(3-\varepsilon) \cdot D$. Otherwise, the maximum delay of any clustering solution of $G$ must be at least $3 \cdot D$. Thus, the $\left(\frac{3}{2}-\varepsilon\right)$-approximation algorithm solves the instance $I$ of $3 \mathrm{SAT}_{\leq 3, \leq 2}$ exactly.

For the purpose of our next reduction, we state the following problems:

Cubic Monotone 1-In-3SAT (CM 1-IN-3S AT): Given a 3-CNF formula $\phi$ with $n$ positive variables $x_{1}, \ldots, x_{n}$ and $m$ clauses $C_{1}, \ldots, C_{m}$, such that each variable appears in exactly three clauses, the goal is to check whether $\phi$ has a satisfying assignment such that every clause of $\phi$ has exactly one true literal. Note that even when restricted to planar graphs, CM 1-IN-3SAT is NP-hard [MR01].

$C N_{d e c}\langle N, 2,4\rangle$ : Given a DAG $G=(V, E)$, with $w(v)=1 \forall v \in V, \delta(v)=0 \forall v \in V$, maximum degree $\Delta=4$, constants $d$ and $D$, cluster capacity $M=2$, and a positive integer $k$, decide whether we can partition $V$ into clusters such that the weight of each cluster is bounded by $M$, and the maximum delay-length of any path from a source to a sink of $G$ is at most $k$. 
By further constraining the clustering of every sink, we consider the following modification of $C N_{d e c}\langle N, 2,4\rangle$ :

$C N_{d e c}^{\prime}\langle N, 2,4\rangle$ : Given a DAG $G=(V, E)$, with $w(v)=1 \forall v \in V, \delta(v)=0 \forall v \in V$, maximum degree $\Delta=4$, constants $d$ and $D$, cluster capacity $M=2$, and a positive integer $k$, decide whether we can partition $V$ into clusters such that the weight of each cluster is bounded by $M$, every sink is clustered with one immediate predecessor, their other immediate predecessors are clustered alone, and the maximum delay-length of any path from a source to a sink of $G$ is at most $k$.

Theorem 3.1.10. $C N_{d e c}^{\prime}\langle N, 2,4\rangle$ is NP-complete.

Proof. It is clear that $C N_{d e c}^{\prime}\langle N, 2,4\rangle$ is in NP. This follows from the well-known fact that a maximum weighted path in an edge-weighted DAG can be found in polynomial time [CLRS09]. Moreover, we can easily verify that the other constraints are met.

In order to establish NP-hardness of $C N_{d e c}^{\prime}\langle N, 2,4\rangle$, we present a reduction from CM 1-IN-3SAT. Let each variable $x_{i}(1 \leq i \leq n)$ be represented by a variable gadget as shown in Figure 3.12(a), Let each clause $C_{j}(1 \leq j \leq m)$ be represented by a clause gadget as shown in Figure 3.12(b). Consider the clause gadget's underlying undirected graph, and let $Y_{j 1}, Y_{j 2}, Y_{j 3}(1 \leq j \leq m)$ denote the three 4-cycles, respectively. If a variable $x_{i}$ is the $1 \mathrm{st}, 2 \mathrm{nd}$, or 3 rd literal of a clause $C_{j}$, then the corresponding vertex labeled $x_{i}$ is connected to a sink labeled $C_{j}$ through some vertices, including the pair $\left\{y_{j 1}, z_{j 1}\right\},\left\{y_{j 2}, z_{j 2}\right\}$, or $\left\{y_{j 3}, z_{j 3}\right\}$, respectively.

We now construct an instance $I^{\prime}$ of $C N_{d e c}^{\prime}\langle N, 2,4\rangle$ as shown in Figure 3.13. The resulting DAG $G$ represents a combinatorial circuit. Let $U$ denote the set of all vertices labeled $x_{i}(1 \leq i \leq n)$. There are $n$ sources labeled $S_{i}$ and $m$ sinks labeled $C_{j}(1 \leq j \leq m)$. They are connected through some vertices in $U$ and $3 \cdot m$ sets of vertices including $\left\{y_{j p}, w_{j p}, z_{j p}\right\}(1 \leq j \leq m, 1 \leq p \leq 3)$. Each $y_{j p}$ is connected to exactly one variable gadget. Every vertex has a weight of 1 . We set $d=0$ and let $D=1$. All vertices are 


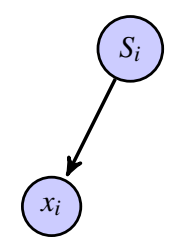

(a) Vari-

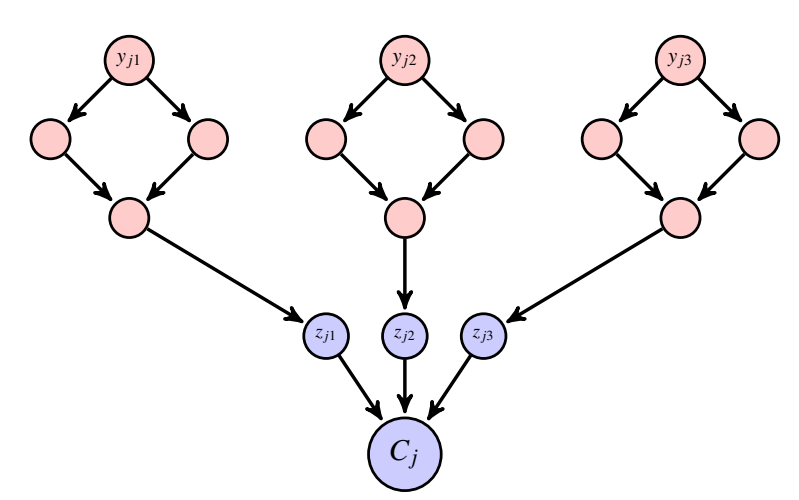

(b) Clause

able

Figure 3.12: Gadgets used to represent variables and clauses.

given a delay of 0 . The cluster capacity $M$ is set to 2 , and we set $k=4$. The description of $I^{\prime}$ is complete.

Observe that $I^{\prime}$ can be constructed from $I$ in polynomial time. In order to complete the proof of the theorem, we show that $I$ is a "yes" instance of CM 1-IN-3SAT, if and only if $I^{\prime}$ is a "yes" instance of $C N_{d e c}^{\prime}\langle N, 2,4\rangle$.

Suppose that $I$ is a "yes" instance of CM 1-IN-3SAT. This means that there exists an assignment of $\phi$ such that every clause has exactly one true literal. If a literal $x_{i}$ is set to true, then the corresponding vertex $x_{i}$ should be clustered alone. However, if a literal $x_{i}$ is set to false, then the corresponding vertex $x_{i}$ is clustered with $S_{i}$. Cluster the vertices of each $Y_{j p}$ so that the intra-cluster edges correspond to a perfect matching. Since each clause $C_{j}$ has exactly one true literal, the vertex $z_{j p}$ along the path corresponding to that true literal is clustered with the sink $C_{j}$. The resulting delay-length of the corresponding source to sink path is $4 \cdot D$. Each remaining $z_{j p}$ not clustered with $C_{j}$ is clustered alone. Observe that the cluster capacity constraint is satisfied, every sink is clustered with one immediate predecessor, their other immediate predecessors are clustered alone, and the delay-length of every path from a source $S_{i}$ to a $\operatorname{sink} C_{j}$ is 4 . This means that $I^{\prime}$ is a "yes" instance of $C N_{d e c}^{\prime}\langle N, 2,4\rangle$.

Conversely, suppose that $I^{\prime}$ is a "yes" instance of $C N_{d e c}^{\prime}\langle N, 2,4\rangle$. This means that 


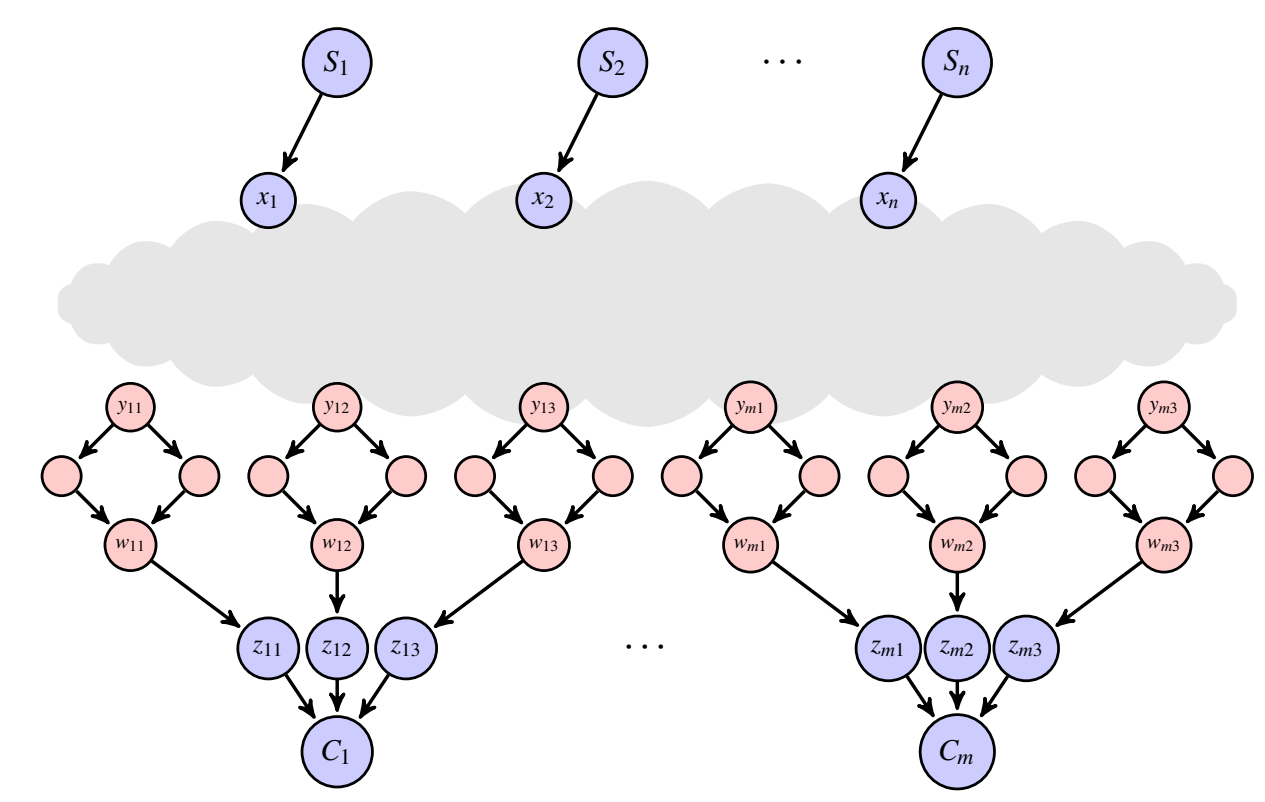

Figure 3.13: Reduction from CM 1-IN-3SAT to $C N_{d e c}^{\prime}\langle N, 2,4\rangle$. The edges connecting variable gadgets to clause gadgets belong in the area with the shaded cloud.

there is a way of partitioning the vertices of $G$ into clusters of capacity $M=2$, such every sink is clustered with one immediate predecessor, their other immediate predecessors are clustered alone, and the delay-length of every path from a source $S_{i}$ to a sink $C_{j}$ is at most 4.

Each sink $C_{j}$ is clustered with one vertex, so the remaining two $z_{j p}$ vertices are clustered alone. Observe that any source to sink path in which a $z_{j p}$ is clustered alone, has a delay-length of at least 4 . Since the delay-length constraint is satisfied, each $S_{i}$ must be clustered with the vertex $x_{i}$ corresponding to a $z_{j p}$ that is clustered alone. Also, the vertices of the set $Y_{j p}$ must be clustered in such a way that the intra-cluster edges form a perfect matching. Take each literal that corresponds to a vertex $x_{i}$ clustered with a source and set its value to false. Now, notice that each $z_{j p}$ along a path in which vertex $x_{i}$ is clustered alone, must be clustered with the sink $C_{j}$. Otherwise, the delay-length of the path would be strictly greater than $4=k$. Take each literal $x_{i}$ that corresponds to a vertex $x_{i}$ clustered alone and set its value to true. Notice that exactly one true 
literal appears in every clause. Thus, a satisfying clustering for $G$ yields a satisfying assignment for $\phi$. Hence, $I$ is a "yes" instance of CM 1-IN-3SAT.

Theorem 3.1.11. $C N\langle N, 2,3\rangle$ is NP-hard.

Proof. We recall $\mathrm{CN}\langle N, 2,3\rangle$ as follows: Given a DAG $G=(V, E)$, with $w(v)=1 \forall v \in$ $V, \delta(v)=0 \forall v \in V$, maximum degree $\Delta=3$, constants $d$ and $D$, cluster capacity $M=2$, and a positive integer $k$, the goal is to partition $V$ into clusters such that the weight of each cluster is bounded by $M$, and the maximum delay-length of any path from a source to a sink of $G$ is minimized.

In order to establish NP-hardness of $\mathrm{CN}\langle N, 2,3\rangle$, we present a reduction from $3 \mathrm{SAT}_{\leq 3, \leq 2}$. For that purpose, we recall $3 \mathrm{SAT}_{\leq 3, \leq 2}$ as follows: Given a $3-\mathrm{CNF}$ formula $\phi$ with $n$ variables $u_{1}, \ldots, u_{n}$ and $m$ clauses $C_{1}, \ldots, C_{m}$, such that each variable occurs at most three times and each literal occurs at most twice, the goal is to check whether $\phi$ has a satisfying assignment. Note that the requirement that each clause has exactly three literals is relaxed in this restriction of $3 \mathrm{SAT}$. Any variable, say $u_{i}$, with $q$ occurrences (for some $q>3$ ) can be replaced with $q$ new variables $w_{1}, \ldots, w_{q}$. The clauses $\left(\bar{w}_{1} \vee w_{2}\right) \wedge\left(\bar{w}_{2} \vee w_{3}\right) \wedge\left(\bar{w}_{q} \vee w_{1}\right)$ can then be added to $\phi$ to ensure that the $q$ new variables retain the truth assignment of the original variable $u_{i}$ [Pap94].

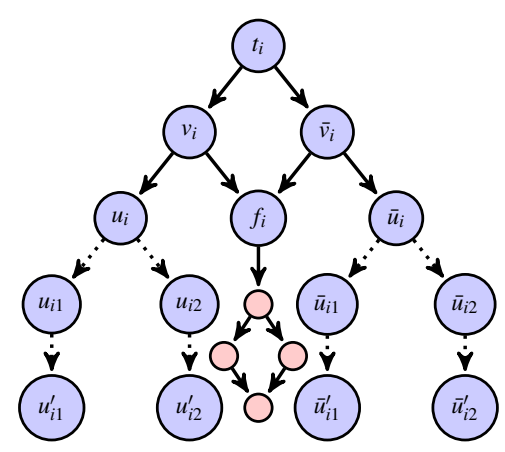

(a) $i$-th variable gadget

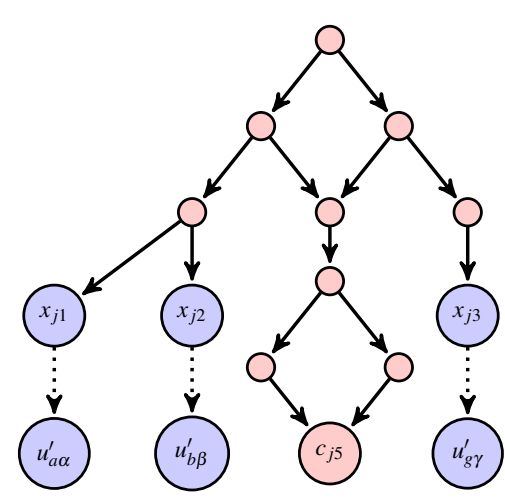

(b) $j$-th clause gadget

Figure 3.14: Gadgets used to represent variables and clauses. 
Given an instance $I$ of $3 \mathrm{SAT}_{\leq 3, \leq 2}$, we construct an instance $I^{\prime}$ of $\mathrm{CN}\langle N, 2,3\rangle$. Let each variable $u_{i}(1 \leq i \leq n)$ be represented by a variable gadget as shown in Figure 3.14(a), where the dashed arrows indicate possible successors. Note that since each variable $u_{i}$ occurs at most three times, then the size of the neighborhood of $\left\{u_{i}, \bar{u}_{i}\right\}$ is at most three. Let each clause $C_{j}(1 \leq j \leq m)$ be represented by a clause gadget as shown in Figure 3.14(b) A set of edges also connects clause gadgets to variable gadgets. For example, if the $p$-th literal of clause $C_{j}$ is the $\alpha$-th occurrence of some literal $u_{a}$, where $p \in\{1,2,3\}, \alpha \in\{1,2\}$ and $a \in\{1, \ldots, n\}$, then we add edge $\left(x_{j p}, u_{a \alpha}^{\prime}\right)$. Every vertex has a weight of 1 . We set $d=0$ and let $D$ be any positive integer. All vertices are given a delay of 0 . The cluster capacity $M$ is set to 2 , and we set $k=3 \cdot D$. The description of $I^{\prime}$ is complete.

Observe that $I^{\prime}$ can be constructed from $I$ in polynomial time. To complete the proof of the theorem, we show that $I$ is a "yes" instance of $3 \mathrm{SAT}_{\leq 3, \leq 2}$ if and only if $I^{\prime}$ is a "yes" instance of $\mathrm{CN}\langle N, 2,3\rangle$.

Suppose that $I$ is a "yes" instance of $3 \mathrm{SAT}_{\leq 3, \leq 2}$. This means that there exists an assignment of $\phi$ such that every clause has at least one true literal. If literal $u_{i}$ (or $\bar{u}_{i}$ ) is set to true, then we cluster the vertices as follows:

1. $t_{i}$ is clustered with $v_{i}$ and $f_{i}$ is clustered with $\bar{v}_{i}$ (or $t_{i}$ is clustered with $\bar{v}_{i}$ and $f_{i}$ is clustered with $v_{i}$ ).

2. For each $r \in\{1,2\}, \bar{u}_{i r}$ is clustered with $\bar{u}_{i r}^{\prime}$ (or $u_{i r}$ is clustered with $u_{i r}^{\prime}$ ).

3. If the $r$-th occurrence of literal $u_{i}$ (or $\bar{u}_{i}$ ) is the $p$-th literal of clause $C_{j}$, then $u_{i r}^{\prime}$ (or $\bar{u}_{i r}^{\prime}$ ) is clustered with clause gadget vertex $x_{j p}$, where $p \in\{1,2,3\}$.

4. For any $p$-th literal of clause $C_{j}$ that is set to true, then $x_{j p}$ is clustered with its successor.

5. The successors of the variable gadget vertex $f_{i}$, say $V_{f_{i}}$, are clustered in such a 
way that the edges of the underlying undirected graph of $G\left[V_{f_{i}}\right]$ form a perfect matching.

6. The clause gadget vertex $c_{j 5}$ and its predecessors, say $V_{c_{j 5}}$, are clustered in such a way that the edges of the underlying undirected graph of $G\left[V_{c_{j 5}} \cup c_{j 5}\right]$ form a perfect matching.

7. All other vertices are clustered alone.

Observe that the cluster capacity constraint is satisfied, and the maximum delaylength of any path from a source to a sink is $3 \cdot D$. This means that $I^{\prime}$ is a "yes" instance of $\mathrm{CN}\langle N, 2,3\rangle$.

Conversely, suppose that $I^{\prime}$ is a "yes" instance of $\mathrm{CN}\langle N, 2,3\rangle$. This means that there is a way of partitioning the vertices of $G$ into clusters of capacity $M=2$, such that the delay-length of any path from a source to a sink is at most $3 \cdot D$. Observe that under any partitioning, the delay-length of any path from a source to a sink is at least $3 \cdot D$. In any partitioning with delay-length equal to $3 \cdot D$, we have that either $t_{i}$ is clustered with $v_{i}$ or $t_{i}$ is clustered with $\bar{v}_{i}$, for every $i \in\{1, \ldots, n\}$. Furthermore, in any partitioning with delay-length equal to $3 \cdot D$, there is at least one $x_{j p}$ that must be clustered with its successor. If $t_{i}$ is clustered with $v_{i}$, then for each $r \in\{1,2\}, \bar{u}_{i r}$ must be clustered with $\bar{u}_{i r}^{\prime}$. Set literal $u_{i}$ to true and consider each $u_{i r}^{\prime}$ free. Otherwise, if $t_{i}$ is clustered with $\bar{v}_{i}$, then for each $r \in\{1,2\}, u_{i r}$ must be clustered with $u_{i r}^{\prime}$. Set literal $\bar{v}_{i}$ to true and consider each $\bar{u}_{i r}^{\prime}$ free. Since at least one $x_{j p}$ is clustered with its successor, namely some free vertex. This means that at least one true literal appears in every clause. Thus, a satisfying clustering for $G$ yields a satisfying assignment for $\phi$. Hence, $I$ is a "yes" instance of $3 \mathrm{SAT}_{\leq 3, \leq 2}$.

Corollary 3.1.10. $C N\langle N, 2,3\rangle$ does not admit a $\left(\frac{4}{3}-\varepsilon\right)$-approximation algorithm for any $\varepsilon>0$, unless $\mathbf{P}=\mathbf{N P}$. 
Proof. By way of contradiction, suppose there exists a $\left(\frac{4}{3}-\varepsilon\right)$-approximation algorithm for $\mathrm{CN}\langle N, 2,3\rangle$. We construct a polynomial time algorithm for $3 \mathrm{SAT}_{\leq 3, \leq 2}$ as follows:

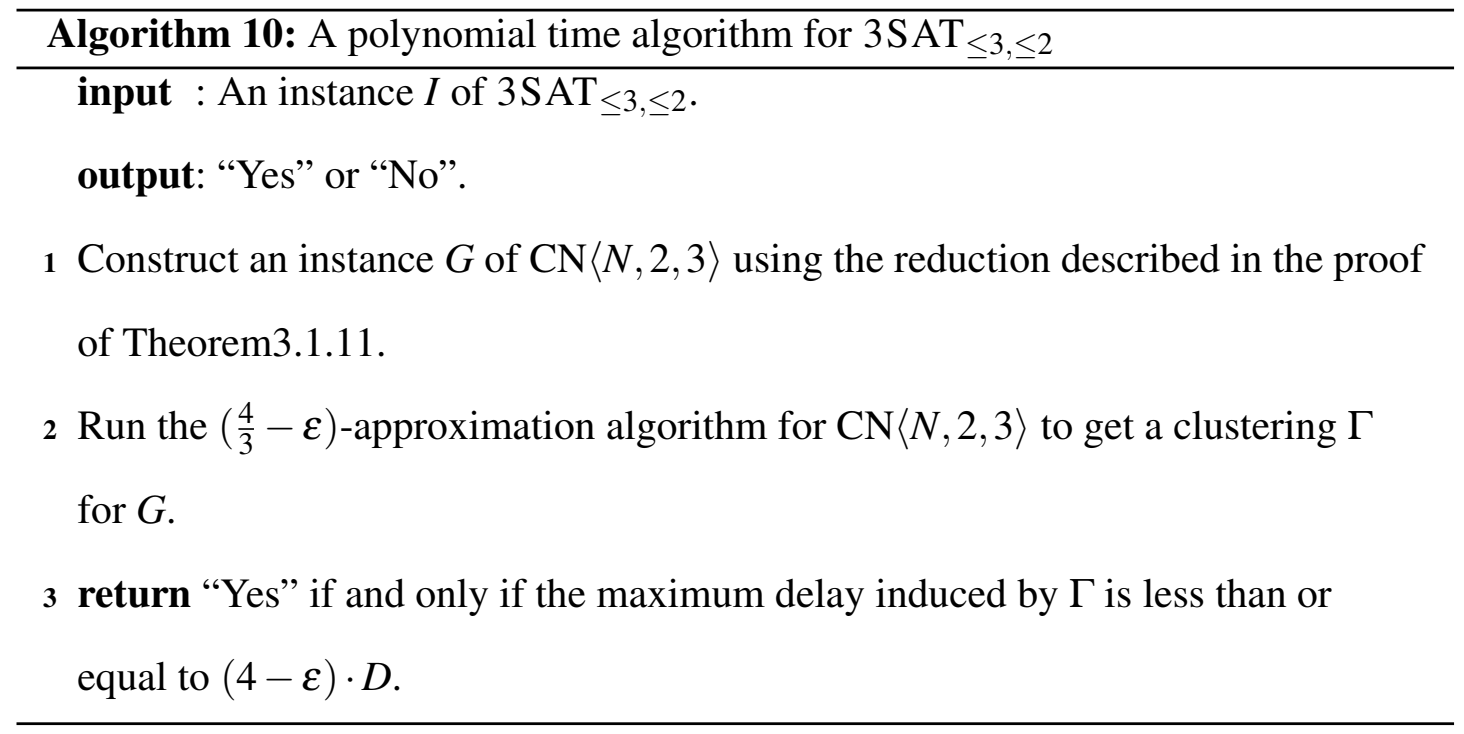

Let $O P T$ denote the delay of the optimal clustering of $G$. If $I$ is a "yes" instance of $3 \mathrm{SAT}_{\leq 3, \leq 2}$, then $O P T \leq 3 \cdot D$. Moreover, the maximum delay of any clustering solution of $G$ returned by the $\left(\frac{4}{3}-\varepsilon\right)$-approximation algorithm for $\mathrm{CN}\langle N, 2,3\rangle$ is at most $(4-\varepsilon) \cdot D$. Otherwise, the maximum delay-length of any clustering solution of $G$ must be at least $4 \cdot D$. Thus, the $\left(\frac{4}{3}-\varepsilon\right)$-approximation algorithm solves the instance $I$ of $3 \mathrm{SAT}_{\leq 3, \leq 2}$ exactly.

\section{Fixed-parameter tractability of $\mathbf{C N}\langle W, M, \Delta\rangle$}

We have shown that $\mathrm{CN}\langle N, 2,3\rangle$ is NP-hard. This result has some direct consequences about the non-fixed parameter tractability of the clustering problem when one is minimizing the delay.

Corollary 3.1.11. The clustering problem $(C N\langle W, M, \Delta\rangle)$ is not fixed-parameter tractable (FPT) with respect to $M, \Delta, d, D, \max _{z \in V} w(z)$ and $w(V)=\sum_{z \in V} w(z)$ unless $\mathbf{P}=\mathbf{N P}$. 
Proof. We prove only for $M$. Others can be done similarly. Assume that the problem is FPT with respect to $M$. This means that the problem is polynomial time solvable when $M=2$. Since by hardness result the $M=2$ case is $\mathbf{N P}$-hard, we have that $\mathbf{P}=\mathbf{N P}$. The proof is complete. 


\section{Chapter 4}

\section{Approximation algorithms}

In this chapter, we propose approximation algorithms for several NP-hard variants of $\mathrm{CN}\langle X, M, \Delta\rangle$ and analyze them.

\subsection{An integer program for $\mathrm{CN}\langle W, M, \Delta\rangle$}

Let $w_{j}$ be the weight of vertex $j$. Define $x_{i j}$ to be an integer variable that is set to 1 , if vertices $i$ and $j$ are in the same cluster, and 0 otherwise. We present the following integer program (IP):

Packing constraints

$$
\begin{gathered}
x_{i i}=1, \quad \forall i \in V \\
\sum_{j=1}^{n} w_{j} \cdot x_{i j} \leq M, \quad \forall i \in V
\end{gathered}
$$

Consistency constraints

$$
\begin{gathered}
x_{i j}=x_{j i}, \quad \forall i, j \in V \\
x_{i k} \geq x_{i j}+x_{j k}-1, \quad \forall i, j, k \in V
\end{gathered}
$$


Condition (4.1) ensures that every vertex is clustered. Condition 4.2 ensures that every cluster has weight at most $M$. Condition (4.3) ensures that either $i$ and $j$ are in the same cluster or they are in different clusters. Likewise, condition (4.4) ensures that if $i$ and $j$ are in one cluster, and $j$ and $k$ are in one cluster, then $i$ and $k$ must be in the same cluster and all clusters are disjoint.

We now come to the objective function. For any vertex $j$, let $\delta_{j}$ be the delay at $j$ in a clustering. This delay is completely dependent upon its predecessors. We can write

$$
\delta_{j}=\max _{i:(i, j) \in E}\left\{\delta_{i}+d \cdot x_{i j}+D \cdot\left(1-x_{i j}\right)\right\}
$$

Hence the function to be minimized is $\delta_{t}$ where $t$ is the sink of the circuit.

Observation 1. The transitivity constraint (i.e., Condition (4.4)) in the IP formulation is necessary in the case even when $M=2$.

Consider the feasible clustering in the small example shown in Figure 4.1 where $M=2$. Notice that in this example, the cluster capacity constraint is satisfied (i.e., $\{s\},\{c\},\{d\},\{a, b\},\{b, t\}$ are all clusters of capacity $\leq 2)$.

Also, the symmetric constraint is satisfied (i.e., $x_{i j}=x_{j i}$ for each pair of vertices).

However, observe that vertex $a$ is clustered with vertex $b$, and vertex $b$ is clustered with vertex $t$, but vertex $a$ is not clustered with vertex $t$. Now, with this 3 rd constraint, the cluster capacity would be violated, and this would not be a feasible clustering. We need the constraint to return a feasible clustering containing a set of disjoint clusters.

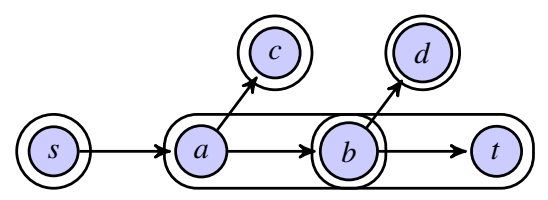

Figure 4.1: Small example to show that the transitivity constraint for the IP is necessary. 


\section{IP reduction}

Let $\mathrm{IP}_{C N\langle W, M, \Delta\rangle}$ denote the IP formulation for $\mathrm{CN}\langle W, M, \Delta\rangle$. To prove that $\mathrm{IP}_{C N\langle W, M, \Delta\rangle}$ is equivalent to $\mathrm{CN}\langle W, M, \Delta\rangle$, we consider their corresponding decision versions. For the decision version $\operatorname{IP}_{C N_{d e c}\langle W, M, \Delta\rangle}$, the goal is to decide whether or not there exists a feasible solution $\mathbf{x} \in \mathbb{B}^{n} \times \mathbb{B}^{n}$ (i.e., some $\mathbf{x} \in \mathbb{B}^{n} \times \mathbb{B}^{n}$ that satisfies every constraint). For the decision version $\mathrm{CN}_{d e c}\langle W, M, \Delta\rangle$, the goal is to decide if there exists a feasible clustering $\Gamma$ (i.e., a disjoint clustering $\Gamma$ of $G$ in which no cluster $C \in \Gamma$ exceeds the cluster capacity $M$ ).

Proposition 4.1.1: $\mathrm{CN}_{d e c}\langle W, M, \Delta\rangle \leq_{P} \operatorname{IP}_{C N_{d e c}\langle W, M, \Delta\rangle} \cdot$

Proof. Given any instance $G=(V, E)$ of $\mathrm{CN}_{d e c}\langle W, M, \Delta\rangle$, we construct an instance $I^{\prime}$ of $\operatorname{IP}_{C N_{\text {dec }}\langle W, M, \Delta\rangle}$. Without loss of generality, we assume that $G$ has a single sink. Let $|V|=n$ and $|E|=m$. Let each edge $(i, j)$ of the underlying undirected graph of $G$ be represented by variable $x_{i j}$. Let the weight of each vertex $j \in V$ be represented by a parameter $w_{j}$. For every $i \in V$, we construct the inequality $w_{1} \cdot x_{i 1}+\ldots+w_{i} \cdot x_{i i}+\ldots+$ $w_{n} \cdot x_{i n} \leq M$. For every pair $i, j \in V$, we construct the equality $x_{i j}=x_{j i}$. For every triple $i, j, k \in V$, we construct the inequality $x_{i k} \geq x_{i j}+x_{j k}-1$. For every $i \in V$, we construct the equality $x_{i i}=1$. The intra- and inter-cluster delays correspond to constants $d$ and $D$, respectively. We let variable $x_{i j}=1$ if vertex $i$ is clustered with vertex $j$, and 0 otherwise. Observe that instance $I^{\prime}$ of $\operatorname{IP}_{C N_{d e c}\langle W, M, \Delta\rangle}$ can be constructed in polynomial time.

Proposition 4.1.2: Let $\mathscr{C}$ be the set of all feasible clusterings of $G$ such that $G$ is an instance of $\mathrm{CN}_{d e c}\langle W, M, \Delta\rangle$. Let $\mathscr{F}$ be the set of all feasible solutions of $I$ such that

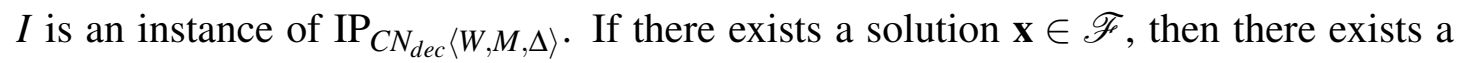
clustering $\Gamma \in \mathscr{C}$.

Proof. Suppose there exists a solution $\mathbf{x}$ of $I$ such that $\mathbf{x} \in \mathscr{F}$. This means that $\mathbf{x} \in$ $\mathbb{B}^{n} \times \mathbb{B}^{n}$, and the constraints 4.2 , (4.3), and (4.4) are all satisfied. We find a clustering 
$\Gamma$ of $G$ as follows: For each $x_{i j}$, such that $x_{i j}=1$ and $i \neq j$, cluster vertices $i$ and $j$ together. Put the remaining vertices into singleton clusters. Since all constraints of the IP are satisfied, then we have a partition $\Gamma$ of the vertex set such that for all clusters $C \in \Gamma$, we have that $w(C) \leq M$. This means that $\Gamma$ is a feasible clustering of $G$. Hence, $\Gamma \in \mathscr{C}$.

Proposition 4.1.3: Let $\mathscr{C}$ be the set of all feasible clusterings of $G$ such that $G$ is an instance of $\mathrm{CN}_{d e c}\langle W, M, \Delta\rangle$. Let $\mathscr{F}$ be the set of all feasible solutions of $I$ such that $I$ is an instance of $\operatorname{IP}_{C N_{d e c}\langle W, M, \Delta\rangle}$. If there exists a clustering $\Gamma \in \mathscr{C}$, then there exists a solution $\mathbf{x} \in \mathscr{F}$.

Proof. Suppose there exists a partition $\Gamma$ of $G$ such that $\Gamma \in \mathscr{C}$. This means that $w(C) \leq$ $M$ for every cluster $C \in \Gamma$. Since $x_{i j}=1$ for all vertices $j$ clustered with vertex $i$, and every vertex is clustered, then $x_{i i}=1$ for all $i$. Hence, constraint (4.1) is satisfied. Moreover, in order to meet the cluster capacity constraint, the sum of the weights of each vertex $j$ clustered with $i$, including the weight of vertex $i$, must not exceed $M$. This means that for each $i$, we satisfy the inequality $w_{1} \cdot x_{i 1}+\ldots+w_{i} \cdot x_{i i}+\ldots+w_{n} \cdot x_{i n} \leq M$. Hence, constraint 4.2 of $\operatorname{IP}_{C N_{d e c}\langle W, M, \Delta\rangle}$ is satisfied.

Next, observe the relationship between the clustering of each pair $i, j \in V$. If vertex $i$ is clustered with vertex $j$, then $x_{i j}=1=x_{j i}$. Otherwise, it is clear that $x_{i j}=0=x_{j i}$. Hence, constraint 4.3 of $\operatorname{IP}_{C N_{d e c}\langle W, M, \Delta\rangle}$ is satisfied.

Finally, notice the relationship between the clustering of any three vertices $i, j, k \in$ $V$. Consider the following cases:

Case 1: If vertex $i$ is clustered with vertex $j$, and vertex $j$ is clustered with vertex $k$, then vertex $i$ is also clustered with vertex $k$. In this case, we have $x_{i j}=1, x_{j k}=1$, and 
$x_{i k}=1$. Observe that

$$
\begin{aligned}
x_{i k} & \geq x_{i j}+x_{j k}-1 \\
\Longrightarrow x_{i k} & \geq 1+1-1 \\
\Longrightarrow x_{i k} & \geq 1 \\
1 & \geq 1
\end{aligned}
$$

Case 2: If vertex $i$ is not clustered with vertex $j$, and vertex $j$ is clustered with vertex $k$, then vertex $i$ cannot be clustered with vertex $k$. In this case, we have $x_{i j}=0, x_{j k}=1$, and $x_{i k}=0$. Observe that

$$
\begin{aligned}
x_{i k} & \geq x_{i j}+x_{j k}-1 \\
\Longrightarrow x_{i k} & \geq 0+1-1 \\
\Longrightarrow x_{i k} & \geq 0 \\
0 & \geq 0
\end{aligned}
$$

Case 3: If vertex $i$ is clustered with vertex $j$, and vertex $j$ is not clustered with vertex $k$, then vertex $i$ cannot be clustered with vertex $k$. In this case, we have $x_{i j}=1, x_{j k}=0$, and $x_{i k}=0$. Observe that

$$
\begin{aligned}
x_{i k} & \geq x_{i j}+x_{j k}-1 \\
\Longrightarrow x_{i k} & \geq 1+0-1 \\
\Longrightarrow x_{i k} & \geq 0 \\
0 & \geq 0
\end{aligned}
$$

Case 4: If vertex $i$ is not clustered with vertex $j$, and vertex $j$ is not clustered with vertex $k$, then vertex $i$ can either be clustered with vertex $k$ or not. In this case, we have $x_{i j}=0$, 
$x_{j k}=0$, and $\left(x_{i k}=1\right.$ or $\left.x_{i k}=0\right)$. Observe that

$$
\begin{aligned}
& x_{i k} \geq x_{i j}+x_{j k}-1 \\
\Longrightarrow & x_{i k} \geq 0+0-1 \\
\Longrightarrow & x_{i k} \geq-1 \\
0 \geq & -1 \text { and } 1 \geq-1
\end{aligned}
$$

Hence, constraint (4.4) of $\operatorname{IP}_{C N_{d e c}\langle W, M, \Delta\rangle}$ is satisfied.

Since every constraint of $\operatorname{IP}_{C N_{d e c}\langle W, M, \Delta\rangle}$ is satisfied, then there exists a solution $\mathbf{x} \in$ $\mathscr{F}$.

Corollary 4.1.1. There exists a solution $\mathbf{x} \in \mathscr{F}$ if and only if there exists a clustering $\Gamma \in \mathscr{C}$. In other words, there exists a bijective function $f: \mathscr{F} \rightarrow \mathscr{C}$ such that $f(\mathbf{x})=\Gamma$.

\subsection{An LP-rounding algorithm for $\mathbf{C N}\langle N, 2, \Delta\rangle$}

In this section, we present an LP-rounding algorithm for $\mathrm{CN}\langle N, 2, \Delta\rangle$. We make use of the linear programming relaxation of the IP formulated in Section 4.1 .

Let $\operatorname{LP}_{C N\langle N, 2, \Delta\rangle}$ be the linear programming relaxation obtained from $\operatorname{IP}_{C N\langle W, M, \Delta\rangle}$ when vertices are unweighted and $M=2$ (i.e., the problem restricted to $\operatorname{CN}\langle N, 2, \Delta\rangle$ ). by replacing its $0-1$ integrality constraints for $x_{i j}$ with $x_{i j} \in[0,1]$. 


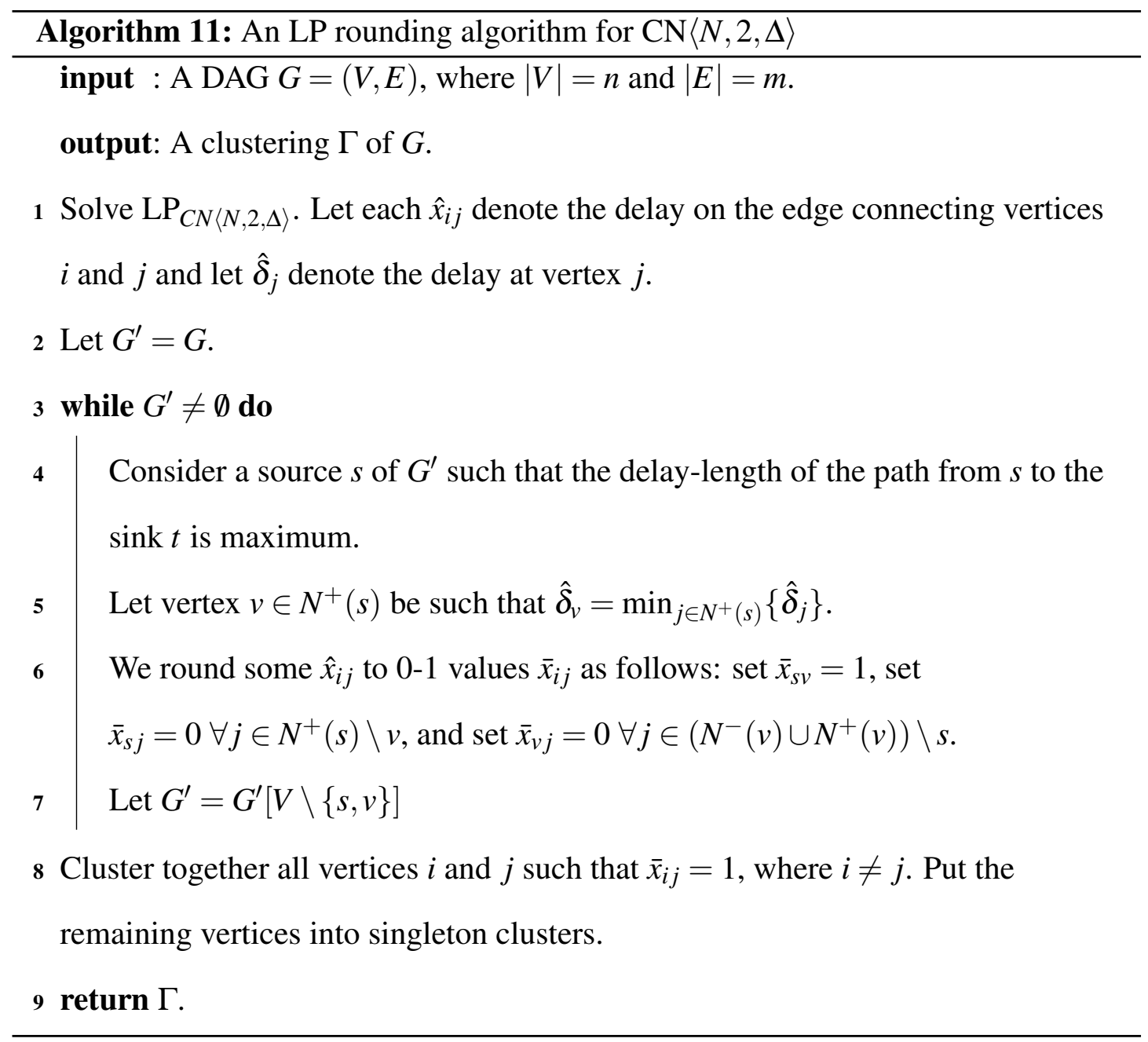

Corollary 4.2.1. Algorithm 11 runs in time $O\left(T_{L P}(n, m)\right)$, where $T_{L P}(n, m)$ is the running time of the fastest linear programming algorithm for solving $L P_{C N\langle N, 2, \Delta\rangle}$.

Theorem 4.2.1. Algorithm 11 is a 2-approximation algorithm.

Proof. Let $Q$ be a path of $G$ from a source to the $\operatorname{sink} t$ with maximum delay-length. Let $O P T$ be the delay of an optimal clustering of $G$. This means that $O P T$ is the sum of the fractional intra- and inter-cluster delays of the edges along $Q$. Let $A L G$ be the delay of the clustering of $G$ returned by Algorithm 11 . Since the algorithm returns a solution with an integral delay, notice that for each intra-cluster edge $(i, j) \in Q$, the delay is increased by $d \cdot\left(1-\hat{x}_{i j}\right)$. Moreover, for each inter-cluster edge $(i, j) \in Q$, the 
delay is decreased by $D \cdot\left(1-\hat{x}_{i j}\right)$. Hence,

$$
\begin{aligned}
\frac{A L G}{O P T} & \leq \frac{\sum_{(i, j) \in Q} d \cdot \hat{x}_{i j}+D \cdot\left(1-\hat{x}_{i j}\right)+\sum_{(i, j) \in Q} d \cdot\left(1-\hat{x}_{i j}\right)-D \cdot\left(1-\hat{x}_{i j}\right)}{\sum_{(i, j) \in Q} d \cdot \hat{x}_{i j}+D \cdot\left(1-\hat{x}_{i j}\right)} \\
& =1+\frac{\sum_{(i, j) \in Q} d \cdot\left(1-\hat{x}_{i j}\right)-D \cdot\left(1-\hat{x}_{i j}\right)}{\sum_{(i, j) \in Q} d \cdot \hat{x}_{i j}+D \cdot\left(1-\hat{x}_{i j}\right)} \\
& \leq 1+\frac{\sum_{(i, j) \in Q} d \cdot\left(1-\hat{x}_{i j}\right)}{\sum_{(i, j) \in Q} d \cdot \hat{x}_{i j}+D \cdot\left(1-\hat{x}_{i j}\right)} \\
& \leq 1+\frac{\sum_{(i, j) \in Q} D \cdot\left(1-\hat{x}_{i j}\right)}{\sum_{(i, j) \in Q} d \cdot \hat{x}_{i j}+D \cdot\left(1-\hat{x}_{i j}\right)} \\
& =2-\frac{\sum_{(i, j) \in Q} d \cdot \hat{x}_{i j}}{\sum_{(i, j) \in Q} d \cdot \hat{x}_{i j}+D \cdot\left(1-\hat{x}_{i j}\right)}
\end{aligned}
$$

Corollary 4.2.2. Algorithm 11 has approximation factor strictly less than 2 when the intra-cluster delay $d>0$.

\subsection{A 3-approximation algorithm for $\mathbf{C N}\langle N, 2, \Delta\rangle$}

In this section, we present a combinatorial 3-approximation algorithm for $\mathrm{CN}\langle N, 2, \Delta\rangle$. Our algorithm makes use of the fact that there is a polynomial-time algorithm for finding a path with a maximum number of edges in DAGs. In each iteration, the algorithm picks a path $P$ with a maximum number of edges. Then it considers the central edge $e=(u, v)$ of $P$, and puts $u$ and $v$ in the same cluster. After that $u$ and $v$ are removed from $G$. The algorithm iterates until all edges of the input DAG are exhausted.

Theorem 4.3.1. Algorithm 12 is a 3-approximation algorithm for $C N\langle N, 2, \Delta\rangle$.

Proof. For a path $P$, let $l(P)$ be the length of $P$ (i.e., the number of edges of $P$ ). More- 


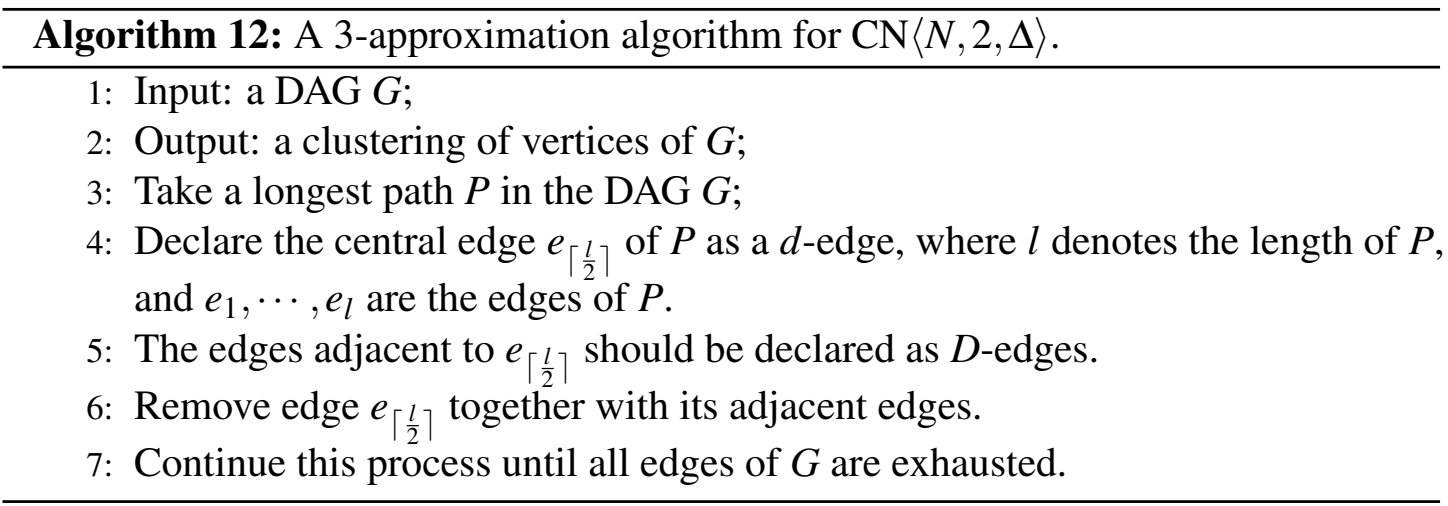

over, let

$$
l=\max _{P} l(P) .
$$

So, $l$ denotes the length of a longest path of $G$.

The following shows a lower bound for $O P T$, where $O P T$ is the delay of the optimal clustering of $G$ when $M=2$.

$$
O P T \geq\left\lceil\frac{l(P)}{2}\right\rceil \cdot d+\left\lfloor\frac{l(P)}{2}\right\rfloor \cdot D
$$

Since $P$ represents any path, then the above inequality must also be true for the longest path. Thus,

$$
O P T \geq\left\lceil\frac{l}{2}\right\rceil \cdot d+\left\lfloor\frac{l}{2}\right\rfloor \cdot D .
$$

Now, let us estimate $A L G$, where $A L G$ is the delay of the clustering found by the algorithm. We will consider 3 cases.

Case 1: $l=1$. Then it can be easily seen that $A L G=O P T$. 
Case 2: $l$ is even. Then

$$
\begin{aligned}
A L G & \leq l \cdot D \\
& \leq 2 \cdot\left(\left\lceil\frac{l}{2}\right\rceil \cdot d+\left\lfloor\frac{l}{2}\right\rfloor \cdot D\right) \\
& \leq 2 \cdot O P T \\
& <3 \cdot O P T .
\end{aligned}
$$

Case 3: $l$ is odd and $l \geq 3$. Then

$$
\begin{aligned}
A L G & \leq l \cdot D \\
& \leq 3 \cdot \frac{l-1}{2} \cdot D \\
& =3 \cdot\left\lfloor\frac{l}{2}\right\rfloor \cdot D \\
& \leq 3 \cdot\left(\left\lceil\frac{l}{2}\right\rceil \cdot d+\left\lfloor\frac{l}{2}\right\rfloor \cdot D\right) \\
& \leq 3 \cdot O P T .
\end{aligned}
$$

The proof of the theorem follows.

Figure 4.2 shows an example of a DAG for which the algorithm achieves an approximation factor of 3 .

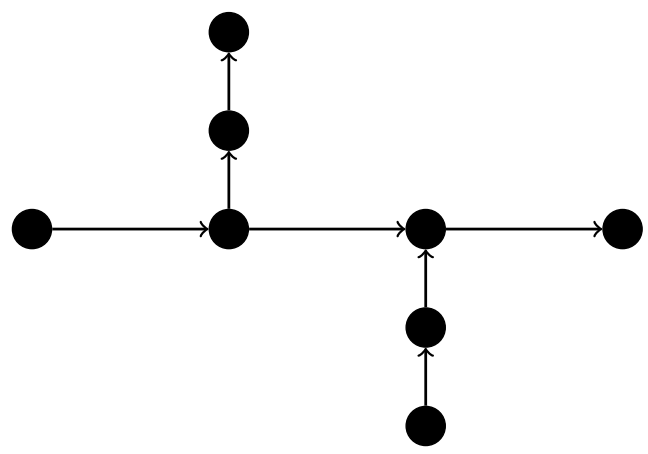

Figure 4.2: A DAG which obtains a factor 3 approximation. 
Observe that in this example, $O P T=2 \cdot d+D$ (see Figure 4.3(a) and $A L G=3 \cdot D$ (see Figure 4.3(b) . Hence, if $d=0$, we have $A L G=3 \cdot O P T$.

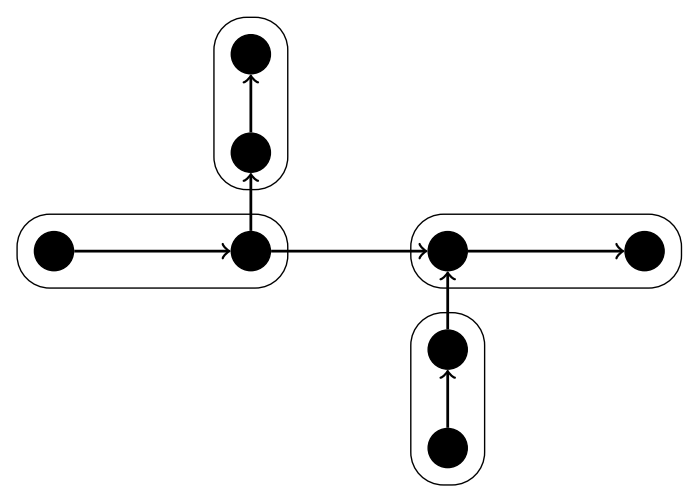

(a) An optimal clustering

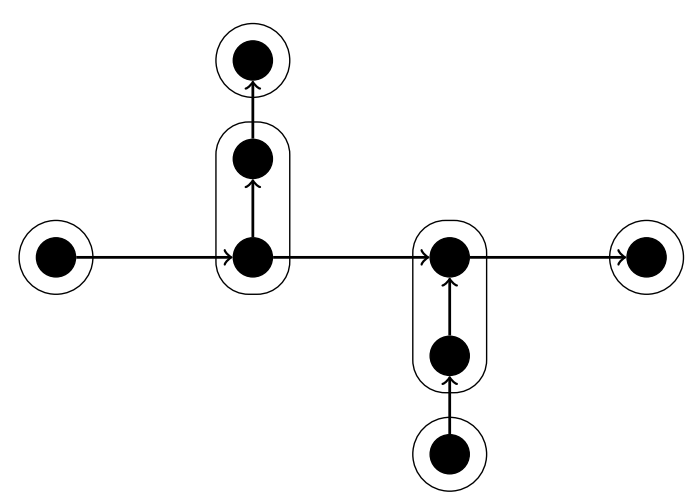

(b) A worst-case clustering by the algorithm

Figure 4.3: An optimal clustering and a worst-case clustering

\subsection{An improved 2-approximation algorithm for $\mathbf{C N}\langle N, 2, \Delta\rangle$}

In this section, we present a 2-approximation algorithm for $\mathrm{CN}\langle N, 2, \Delta\rangle$. Our algorithm makes use of the fact that there is a polynomial time algorithm for finding a path with maximum edge-weight in DAGs. The algorithm tries to construct a so-called dominating matching of the input DAG $G$. We prove that this algorithm has a performance 
ratio of 2 .

We start with the following:

Definition 4.4.1. A matching in a DAG $G$ is a collection of edges that do not share a vertex. A matching of a DAG $G$ is perfect if any vertex of $G$ is incident to an edge from the matching.

Let $G=(V, A)$ be a DAG. Clearly, any perfect matching of $G$ contains exactly $\frac{|V|}{2}$ edges. Let $l$ be the length of a longest path in $G$.

Definition 4.4.2. A matching $I$ in $G$ is dominating, if every longest path of $G$ contains more than $\frac{l}{2}$ edges of $I$.

It is easy to see that if $G$ contains a dominating matching, then $l$ has to be odd.

Lemma 4.4.1. Let $G=(V, A)$ be a DAG and let $l$ be the length of a longest path in $G$. There is a polynomial algorithm which decides whether $G$ has a dominating matching and finds one if it exists.

Proof. We may assume that $G$ is connected, i.e., its undirected underlying graph is connected. If $l$ is even, then there is no dominating matching. So we may assume that $l$ is odd.

Let $I=\emptyset$. Construct a longest path $P$ in $G$ and add odd edges to $I$. Construct a set $S$ initially consisting of the edges of $P$. Note that $I$ is a perfect matching in $G[S]$, the subgraph of $G$ induced by $S$. This property of $I$ in $G[S]$ is maintained.

Consider an edge $a$ in $A \backslash S$ such that only one end-vertex of $a$ is in $S$. If there is not such an edge $a$, remove the edges of $A \backslash S$ from $G$. Also, remove the resulting isolated vertices of $G$. If $a$ does not belong to a longest path of $G$, delete it from $G$ and remove the resulting isolated vertices from $G$. Otherwise, let $Q$ be a longest path of $G$ passing through $a$. Add all odd edges of $Q$ to $I$. If an edge of $Q$ assigned to $I$ is incident to an edge of $I$ in $G[S]$, then we have that $G$ has no dominating matching. Otherwise, add all 
edges of $Q$ to $S$ and observe that $I$ is a perfect matching in $G[S]$. Continue for as long as $A \neq S$.

Now we have that $A=S$ and $I$ is a perfect matching in $G[S]$. Since every longest path of $G$ must start from an edge in $I$ and end with an edge of $I$, the only possibility for a longest path to contain at most $\frac{l}{2}$ edges of $I$ is if it contains two consecutive edges that are not from $I$. Thus, we do the following. Consider every pair of edges not from I forming a directed path of length 2 and check whether the pair is on any longest path of $G$. If so, $G$ has no dominating matching. Otherwise, $I$ is a dominating matching.

The above proof is an algorithm which runs in polynomial time. The proof of the lemma is complete.

Using Lemma 4.4.1, we obtain a 2-approximation algorithm for $\mathrm{CN}\langle N, 2, \Delta\rangle$.

Theorem 4.4.1. The problem $C N\langle N, 2, \Delta\rangle$ admits a 2-approximation algorithm.

Algorithm 13: A 2-approximation algorithm for $\mathrm{CN}\langle N, 2, \Delta\rangle$.

1: Input: a DAG $G$;

2: Output: a clustering of vertices of $G$;

3: Check whether $G$ has a dominating matching.

4: If it does not, return an arbitrary feasible clustering of $G$ (for example, put each vertex in a separate cluster).

5: If $G$ contains a dominating matching $I$, then for each edge $e=u v \in I$, put $u$ and $v$ in the same cluster, and put the remaining vertices in a separate cluster.

6: Output the resulting clustering of $G$.

Proof. Consider Algorithm 13, which is a generic algorithm for the problem. For a path $P$, let $l(P)$ be the length of $P$ (i.e., the number of edges of $P$ ). Moreover, let $l$ denote the length of a longest path of $G$.

The following shows a lower bound for $O P T$, where $O P T$ is the delay of the optimal clustering of $G$ when $M=2$.

$$
O P T \geq\left\lceil\frac{l(P)}{2}\right\rceil \cdot d+\left\lfloor\frac{l(P)}{2}\right\rfloor \cdot D .
$$


Since $P$ represents any path, then the above inequality must also be true for the longest path. Thus,

$$
O P T \geq\left\lceil\frac{l}{2}\right\rceil \cdot d+\left\lfloor\frac{l}{2}\right\rfloor \cdot D
$$

Now, let us estimate $A L G$, where $A L G$ is the delay of the clustering found by the algorithm. We consider 2 cases.

Case 1: $G$ has no dominating matching. Then if $l$ is even, we have

$$
\begin{aligned}
A L G & \leq l \cdot D \\
& \leq 2 \cdot\left(\left\lceil\frac{l}{2}\right\rceil \cdot d+\left\lfloor\frac{l}{2}\right\rfloor \cdot D\right) \\
& \leq 2 \cdot O P T .
\end{aligned}
$$

On the other hand, if $l$ is odd, then since $G$ has no dominating matching, we have

$$
O P T \geq\left\lfloor\frac{l}{2}\right\rfloor \cdot d+\left\lceil\frac{l}{2}\right\rceil \cdot D
$$

Hence

$$
\begin{aligned}
A L G & \leq l \cdot D \\
& \leq 2 \cdot\left(\left\lfloor\frac{l}{2}\right\rfloor \cdot d+\left\lceil\frac{l}{2}\right\rceil \cdot D\right) \\
& \leq 2 \cdot O P T .
\end{aligned}
$$

Case 2: $G$ has a dominating matching $I$. Then since any path of length $l$ has an edge 
from $I$, we have

$$
\begin{aligned}
A L G & \leq d+(l-1) \cdot D \\
& \leq 2 \cdot\left(\left\lceil\frac{l}{2}\right\rceil \cdot d+\left\lfloor\frac{l}{2}\right\rfloor \cdot D\right) \\
& \leq 2 \cdot O P T .
\end{aligned}
$$

The proof of the theorem is complete.

\subsection{A simple $\left(2+\frac{2}{l-1}\right)$-approximation algorithm}

We present a simple approximation algorithm for $\mathrm{CN}\langle N, 2, \Delta\rangle$ as follows:

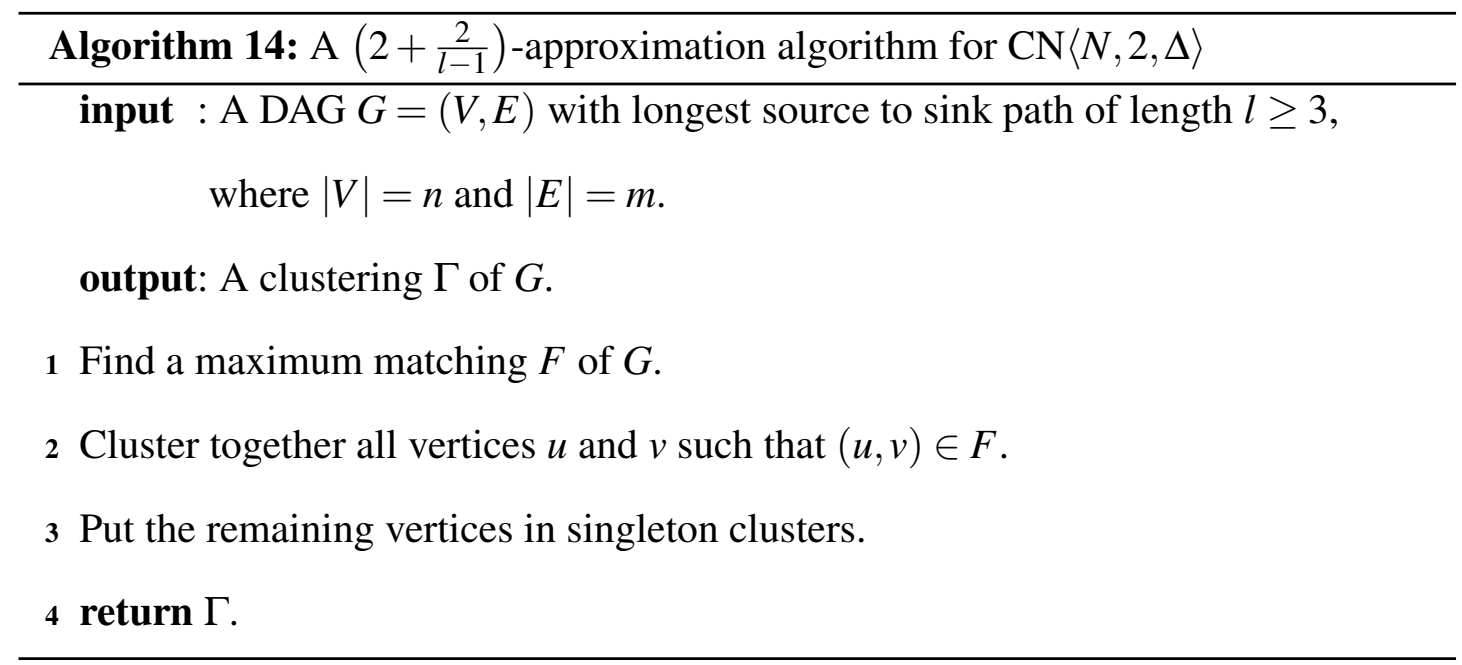

Corollary 4.5.1. Algorithm 14 runs in time $O(\sqrt{n} \cdot m)$.

Theorem 4.5.1. Algorithm 14 is a $\left(2+\frac{2}{l-1}\right)$-approximation algorithm.

Proof. Let $O P T$ be the delay of an optimal clustering of $G$, let $A L G$ be the delay of the clustering found by Algorithm 14 , and let $l$ denote the length of a longest source to sink path in $G$. Observe that when the cluster capacity $M=2$, then $O P T \geq\lceil l / 2\rceil \cdot d+$ $\lfloor l / 2\rfloor \cdot D$, where $d$ and $D$ are the intra- and inter-cluster delays, respectively. Moreover, $O P T \geq\lfloor l / 2\rfloor \cdot D$ when $d=0$. Since there are at most $l$ inter-cluster edges in a clustering 
of $G$, then we have

$$
\begin{aligned}
\frac{A L G}{O P T} & \leq \frac{l \cdot D}{\lfloor l / 2\rfloor \cdot D} \\
& \leq \begin{cases}2, & \text { if } l \text { is even } \\
2+\frac{2}{l-1}, & \text { if } l \text { is odd }\end{cases}
\end{aligned}
$$

Corollary 4.5.2. Let $l$ denote the length of a longest source to sink path in $G$. If $l \geq 3$ and $0<d \ll D<(l+1) \cdot d$, where $d$ and $D$ are the intra- and inter-cluster edges, respectively, then Algorithm 14 returns a clustering with a maximum delay-length that is strictly less than twice the optimum.

Proof. Suppose $l \geq 2$ and $0<d \ll D<(l+1) \cdot d$. Let $O P T$ be the delay of an optimal clustering of $G$ and let $A L G$ be the delay of the clustering found by Algorithm 14 . Observe that when the cluster capacity $M=2$, then $O P T \geq\lceil l / 2\rceil \cdot d+\lfloor l / 2\rfloor \cdot D$. Since there are at most $l$ inter-cluster edges in a clustering of $G$, then we have

$$
\begin{aligned}
\frac{A L G}{O P T} & \leq \frac{l \cdot D}{\lceil l / 2\rceil \cdot d+\lfloor l / 2\rfloor \cdot D} \\
& = \begin{cases}\frac{2 \cdot D}{d+D}, \quad \text { if } l \text { is even } \\
\frac{2 \cdot l \cdot D}{(l+1) \cdot d+(l-1) \cdot D}, \quad \text { if } l \text { is odd }\end{cases} \\
& =\left\{\begin{array}{l}
2-2 \cdot \frac{d}{d+D}, \quad \text { if } l \text { is even } \\
2-2 \cdot \frac{(l+1) \cdot d-D}{(l+1) \cdot d+(l-1) \cdot D}, \quad \text { if } l \text { is odd }
\end{array}\right. \\
& \leq 2-2 \cdot \min \left\{\frac{d}{d+D}, \frac{(l+1) \cdot d-D}{(l+1) \cdot d+(l-1) \cdot D}\right\} \\
& <2
\end{aligned}
$$




\subsection{A parameterized approximation algorithm for}

$$
\mathbf{C N}\langle W, M, \Delta\rangle
$$

Here, we present a parameterized approximation algorithm for $\mathrm{CN}\langle W, M, \Delta\rangle$.

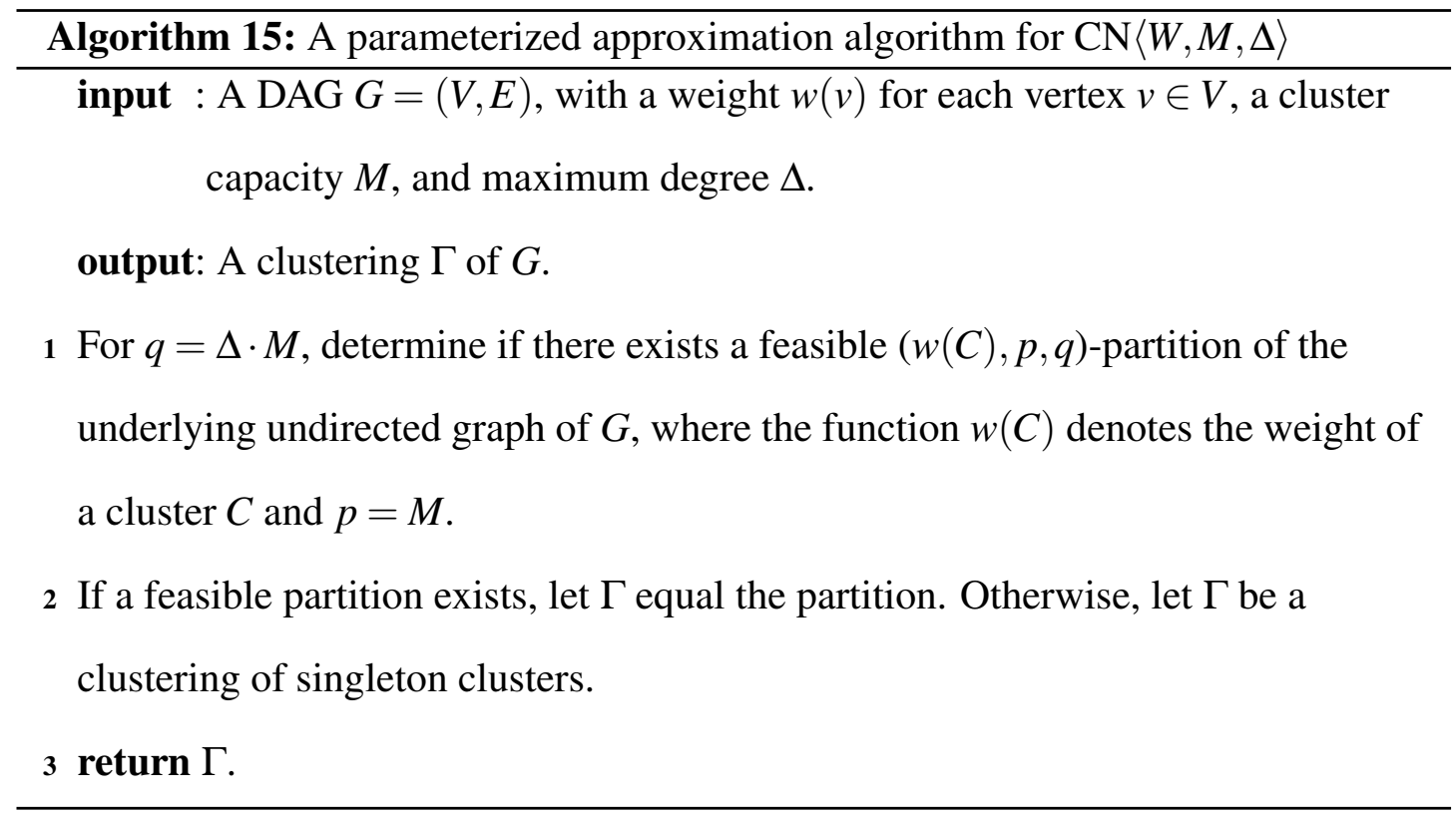

Theorem 4.6.1. $(w(C), p, q)$-PARTITION can be solved in time $2^{O(q)} \cdot|V|^{O(1)}$. $\left[C F K^{+} 15\right.$

Corollary 4.6.1. Algorithm 15 can be solved in time $2^{O(\Delta \cdot M)} \cdot|V|^{O(1)}$.

Theorem 4.6.2. Algorithm 15 is an $\left(M+M^{2}\right)$-factor parameterized approximation algorithm.

Proof. Let $O P T$ be the delay of an optimal clustering of $G$ and let $A L G$ be the delay of the clustering found by Algorithm 15 . Let $l$ denote the length of a longest source to 
sink path in $G$. Observe that

$$
O P T \geq \begin{cases}{[(l+1) / M] \cdot(M-1) \cdot d+[(l+1) / M-1] \cdot D,} & \text { if } M \mid(l+1) \\ \lceil(l+1) / M\rceil \cdot(M-1) \cdot d+\lfloor(l+1) / M\rfloor \cdot D, & \text { if } M \nmid(l+1)\end{cases}
$$

where $d$ and $D$ are the intra- and inter-cluster delays, respectively.

Notice that if $l+1 \leq M$, then all vertices on source to sink paths can be clustered together, and we are done. Therefore, we may assume that $(l+1)>M$. Next, we let $d=0$ and obtain the following:

$$
O P T \geq \begin{cases}{[(l+1) / M-1] \cdot D,} & \text { if } M \mid(l+1) \\ \lfloor(l+1) / M\rfloor \cdot D, & \text { if } M \nmid(l+1)\end{cases}
$$

Since there are at most $l$ inter-cluster edges in any clustering of $G$, then $A L G \leq l \cdot D$. We now consider the following two cases:

Case 5: If $M \mid(l+1)$, then

$$
\begin{aligned}
\frac{A L G}{O P T} & \leq \frac{l \cdot D}{[(l+1) / M-1] \cdot D} \\
& =M+M \cdot\left(\frac{M-1}{l+1-M}\right) \\
& \leq M+M \cdot(M-1) \\
& \leq M+M^{2}
\end{aligned}
$$

The inequality in 4.8 follows from the assumption that $(l+1)>M$, which implies that $l+1-M \geq 1$.

Case 6: If $M \nmid(l+1)$, then $l+1=M \cdot b+r$ for some positive integer $b$ and an integer 
$r \in\{1, \ldots, M-1\}$. This means that

$$
\begin{aligned}
\frac{A L G}{O P T} & \leq \frac{l \cdot D}{\lfloor(l+1) / M\rfloor \cdot D} \\
& =\frac{M \cdot l}{l+1-r} \\
& =M+M \cdot\left(\frac{r-1}{l+1-r}\right) \\
& \leq M+M \cdot\left(\frac{M-1}{l+1-M}\right) \\
& \leq M+M \cdot(M-1) \\
& \leq M+M^{2}
\end{aligned}
$$




\section{Chapter 5}

\section{Exact Exponential Algorithms}

In this chapter, we propose exact exponential algorithms for several NP-hard variants of $\mathrm{CN}\langle X, M, \Delta\rangle$ and analyze them. We assume that $n=|V|$ and $m=|E|$. We also use a modified big-O notation, as defined in [FK10], that suppresses all polynomially bounded factors. For functions $f$ and $g$ we write $f(n)=O(g(n))$ if $f(n)=O(g(n) \operatorname{poly}(n))$, where $\operatorname{poly}(n)$ is a polynomial.

\subsection{Exact exponential algorithms for $\mathbf{C N}\langle W, M, \Delta\rangle$}

Since $\mathrm{CN}\langle W, M, \Delta\rangle$ is a partition problem (see [FK10] for details), we can solve this problem by simply generating all possible partition of the vertices of $G$. As it is stated in [FK10], the number of these partitions it most $n^{n}$, which is roughly $2^{O(n \cdot \log n)}$. Thus, we have:

Observation 2. $C N\langle W, M, \Delta\rangle$ can be solved in time $O^{*}\left(2^{n \cdot \log n}\right)$.

When the vertices of $G$ are partitioned, each edge $e$ of $G$ can have a delay $d$ or $D$. Thus, we have at most $2^{m}$ possible assignments of delays to edges of $G$. If we generate all these $2^{m}$ assignments and for each them check in polynomial time whether it corresponds to a feasible clustering and if it is, we compute the optimal delay of $G$, 
we can find an optimal clustering in time $O^{*}\left(2^{m}\right)$. Thus, we have:

Observation 3. $C N\langle W, M, \Delta\rangle$ can be solved in time $O^{*}\left(2^{m}\right)$.

\subsection{Exact exponential algorithms for $\mathbf{C N}\langle N, 2, \Delta\rangle$}

Now, we are going to discuss some ideas that will lead to exact algorithms for some restrictions of $\mathrm{CN}\langle W, M, \Delta\rangle$. The running-time of these algorithms will be smaller than those presented by trivial algorithms in Observations 2 and 3 . We focus on the case $M=2$ which we know to be NP-hard. For this case, we will say that two edges of $G$ are independent if they are not incident to the same vertex. A matching of $G$ is a set of pairwise independent edges of $G$. A matching is maximal if it is not a subset of a larger matching.

Proposition 5.2.1: For any instance of $\mathrm{CN}\langle W, M, \Delta\rangle$ there exists an optimal clustering, such that the edges of $G$ with delay $d$ form a maximal matching of $G$.

Proof. Consider an optimal clustering of $G$. Since $M=2$, we have that any two edges with delay $d$ are independent. Thus, they form a matching $I$. Now, if $I$ is not maximal, then there is an edge $e$, such that $I \cup\{e\}$ is a matching. Put the end-vertices of $e$ to the same cluster. Observe that the resulting clustering is feasible, moreover, its delay does not exceed the delay of the original clustering. Thus, the resulting clustering is again optimal. By continuing this process, we will end-up with a clustering such that the edges of delay $d$ form a maximal matching. The proof is complete.

In order to describe our first algorithm, for each edge $e$ let $N[e]$ be the closed neighborhood of $e$, that is, the set of edges adjacent to $e$ together with $e$.

Proposition 5.2.2: Let $e$ be any edge of the graph $G$. Then there is an optimal clustering of $G$, such that the matching of edges with delay $d$ has non-empty intersection with $N[e]$. 
Proof. Take any optimal clustering such that the edges with delay $d$ do not intersect $N[e]$. Then we can put the end-vertices of $e$ in one cluster, and $e$ will become a $d$-edge. Observe that the resulting clustering is feasible, moreover, its delay does not exceed that of the original clustering. Thus, the resulting clustering is optimal. The proof is complete.

Proposition 5.2.2 prompts the following algorithm for $\mathrm{CN}\langle N, 2, \Delta\rangle$. We can take any maximal matching $I=\left\{e_{1}, \ldots, e_{t}\right\}$, and we can try all possible $t$-tuples of edges $N\left[e_{1}\right] \times \cdots \times N\left[e_{t}\right]$. Since $t \leq \frac{n}{2}$ and for each $j=1, \ldots, t\left|N\left[e_{j}\right]\right| \leq 2 \cdot \Delta-1$, we have that the running-time of this algorithm is bounded by

$$
O^{*}\left(N\left[e_{1}\right] \times \cdots \times N\left[e_{t}\right]\right) \leq O^{*}\left((2 \cdot \Delta-1)^{\frac{n}{2}}\right)=O^{*}\left((\sqrt{2 \cdot \Delta-1})^{n}\right) .
$$

The formal description of the algorithm is (see Algorithm 16).

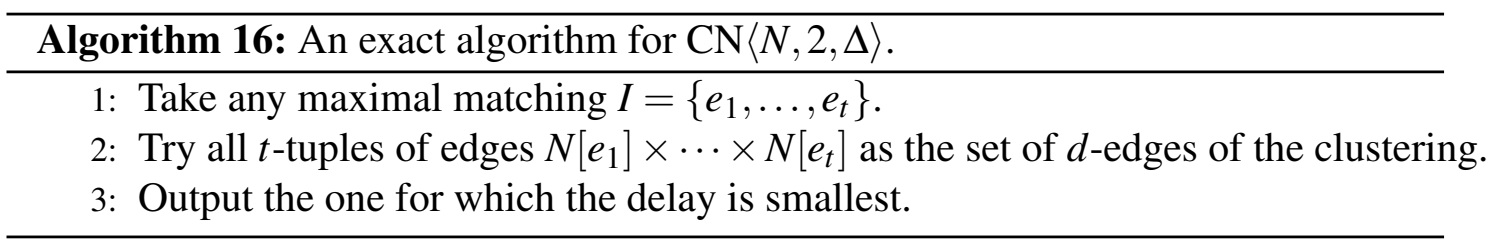

First observe that the maximal matching in step 1 of Algorithm 16 can be constructed in linear time. Also, note that when any vertex of $G$ is of degree 3, the trivial algorithm in Observation 3 runs in time $O^{*}\left(2^{\frac{3 \cdot n}{2}}\right)=O^{*}\left((\sqrt{8})^{n}\right)$, while Algorithm 16 runs in time $O^{*}\left((\sqrt{2 \cdot 3-1})^{n}\right)=O^{*}\left((\sqrt{5})^{n}\right)$.

In order to describe our next algorithm, let us give additional definitions. Two vertices of $G$ are independent, if there is no edge of $G$, whose end-vertices are these two vertices. An independent set is maximal if it is not a subset of a larger independent set. Our next algorithm makes use of the following result:

Theorem 5.2.1. (page 11, [FK10])) Any graph $G$ contains at most $3^{\frac{n}{3}}$ maximal independent set of vertices. Moreover, these independent sets can be enumerated in time 
$O^{*}\left(3^{\frac{n}{3}}\right)$

For each graph $G$ consider its line graph $L(G)$. In $L(G)$ the set of vertices are the edges of $G$, and two vertices of $L(G)$ are adjacent if they share a vertex in $G$. Let us take an arbitrary graph $G$ and construct its line graph $L(G)$. By Theorem 5.2.1, it has at most $3^{\frac{|E|}{3}} \leq 3^{\frac{n \Delta}{6}}$ maximal independent sets. Moreover, these maximal independent sets can be enumerated in time $O^{*}\left(3^{\frac{n \Delta}{6}}\right)$. Observe that any maximal independent set of $L(G)$ is just a maximal matching of $G$. Thus, $G$ has at most $3^{\frac{n \Delta}{6}}$ maximal matchings. We try all these matchings as a candidate for the optimal clustering. Thanks to Proposition 5.2.1. at least one of them should be an optimal clustering. Thus, we can solve the clustering problem in time $O^{*}\left(3^{\frac{n \Delta}{6}}\right)$.

The formal description of the algorithm is (see Algorithm 17).

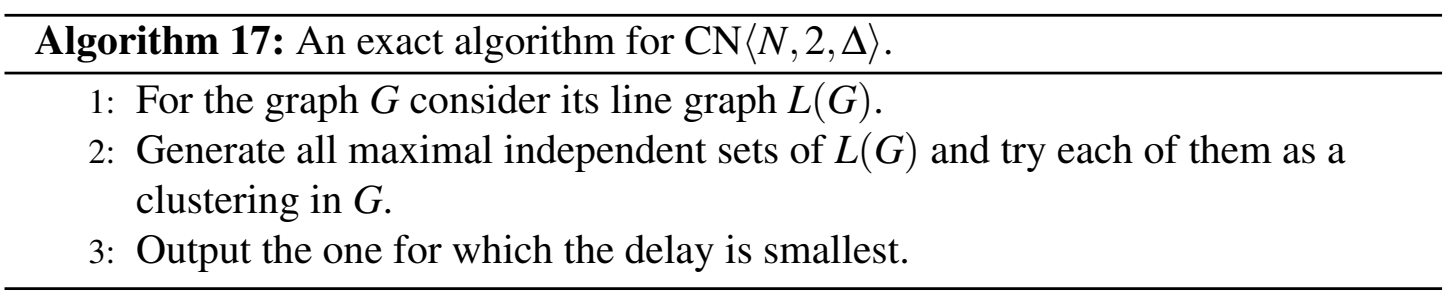

Note that when any vertex of $G$ is of degree 3, Algorithm 16 runs in time $O^{*}\left((\sqrt{2 \cdot 3-1})^{n}\right)=O^{*}\left((\sqrt{5})^{n}\right)$, while Algorithm 17 runs in time $O^{*}\left(3^{\frac{n \Delta}{6}}\right)=$ $O^{*}\left((\sqrt{3})^{n}\right)$.

\subsection{An exact exponential algorithm for $\mathbf{C N}\langle W, M, 3\rangle$}

We also considered the following alternative approach: Given a DAG $G=(V, E)$. Let $G^{\prime}=\left(V^{\prime}, E^{\prime}\right)$, where $V^{\prime}=V$ and $E^{\prime}=\emptyset$. Start with a clustering $\Gamma$ of singleton clusters of $G^{\prime}$. Add an edge $e \in E$ to $E^{\prime}$, such that $e$ belongs to set of edges incident from a source in $G$. Re-cluster $G^{\prime}$ to minimize the maximum delay. Set $\Gamma$ equal to the 
re-clustering. While $E^{\prime} \neq E$, continue to add an edge $e \in E$ to $E^{\prime}$, then re-cluster to minimize the maximum delay of $G^{\prime}$. Return $\Gamma$.

From this approach, we made the following observation.

Observation 4. After adding the last edge, we may not have an optimal clustering. In the worst case, we may have to re-cluster the original graph, even after exhausting all intermediate re-clusterings. Hence, this approach runs in time $O^{*}\left(2^{m}\right)$.

Consider the example of a simple DAG below.

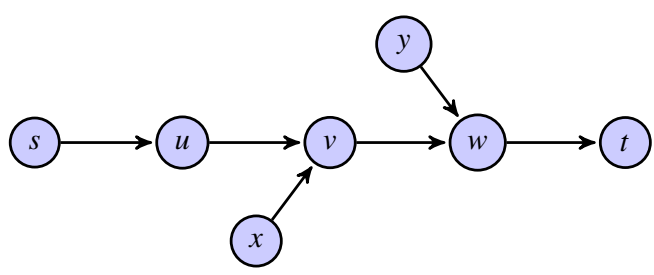

(a) A simple example of a DAG $G$

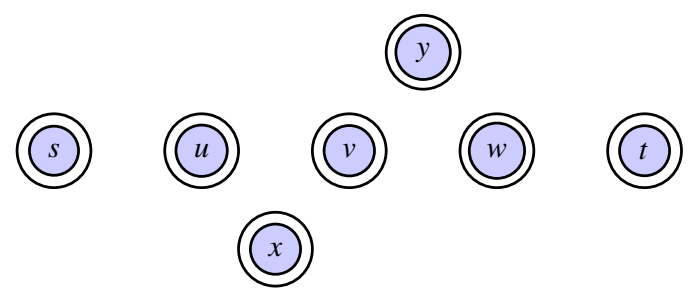

(b) A clustering of singleton clusters

Observe that if we first add edge $(x, v)$, it makes sense to put vertices $x$ and $v$ into a cluster.

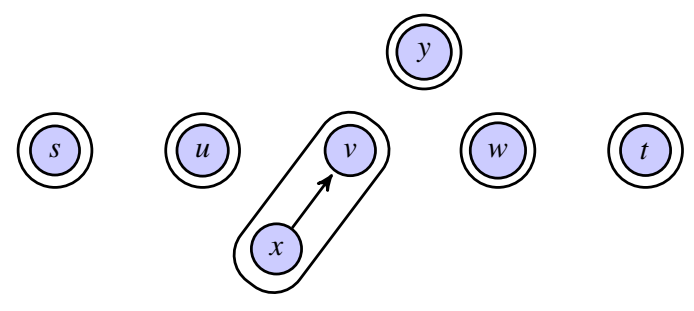

(c) 
Next, suppose that we add edge $(y, w)$. Then it makes sense to put vertices $y$ and $w$ into a cluster.

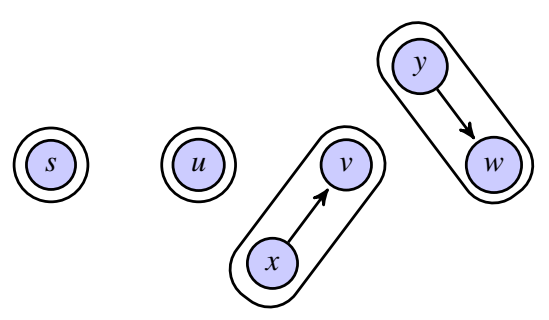

(t)

(d)

Now, suppose that we add edge $(s, u)$. Then it makes sense to put vertices $s$ and $u$ into a cluster.
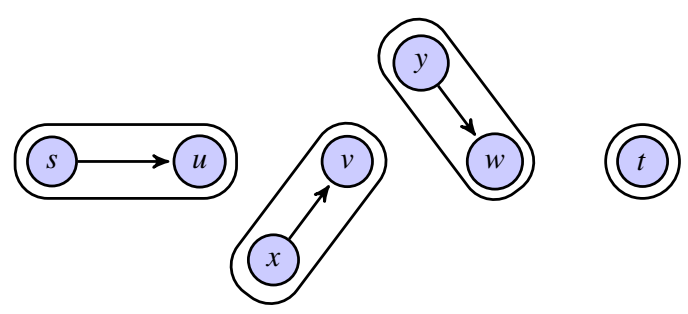

Suppose that we add edge $(w, t)$ next. It does not make sense to re-cluster any other vertices, because at least one vertex on the 2-path must be clustered alone. Thus, keeping vertex $t$ in a singleton cluster will suffice at this point.

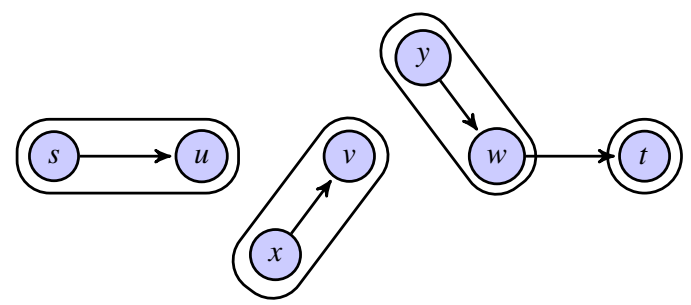

(f) 
Next, if we add edge $(u, v)$, it does not make sense to re-cluster any other vertices. No matter the clustering at this stage, the current maximum delay-length is minimum.

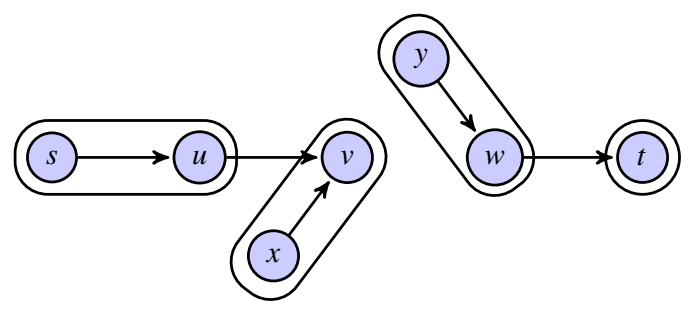

(g)

Finally, we add edge $(v, w)$. Observe that the delay-length of the final clustering of $G$ is $3 \cdot D$. But this is not optimal.

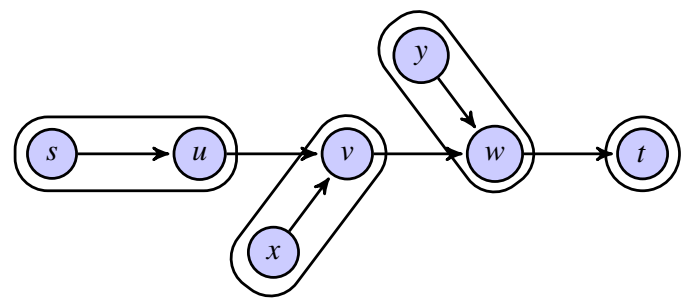

(h)

The clustering of $G$ shown below is an example of an optimal clustering.

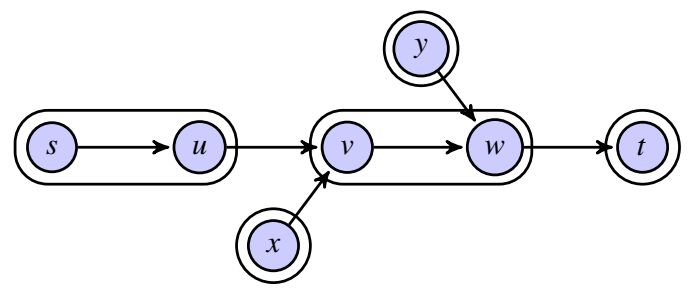

(i) An optimal clustering of $G$ with max delaylength $2 \cdot D$

The previous approach gave us some insights which lead to the next result. 
Corollary 5.3.1. $C N\langle W, M, 3\rangle$ can be solved exactly in time $O\left(4^{n}\right)$.

Proof. Given an instance $G$ of $\mathrm{CN}\langle W, M, 3\rangle$, we partition $V$ into levels $V_{0}, \ldots, V_{l}$, where $l$ is the "height" (as described in [AMY18]) (i.e., the longest length of any path from a source to a sink in $G$ ). Layer $V_{0}$ contains all sources of $G$, and vertex $v \in V \backslash V_{0}$ belongs to the layer whose index $k$ represents the length of the longest path from a source to $v$. Moreover, there exists an edge $(u, v)$ for every vertex $v \in V_{k}(1 \leq k \leq l)$ such that $u \in V_{k-1}$. Observe that $V_{l}$ is the set of sinks in $G$ such that the length from a source to a $\operatorname{sink} t \in V_{l}$ is $l$. Note that such a partition can be found in $O(|V|+|E|)$ time.

Let $\delta_{G\left[V \backslash V_{0}\right]}$ be the maximum delay-length that is minimum over all possible $2^{\left|E\left(G\left[V \backslash V_{0}\right]\right)\right|}$ clusterings of $G\left[V \backslash V_{0}\right]$. Then, we have

$$
\delta_{G}= \begin{cases}\delta_{G\left[V \backslash V_{0}\right]}+d \quad & \text { if } \forall u \in V_{0}, \text { s.t. } u \text { belongs to a path of length } l, \\ & \exists ! v \in V_{1} \text { s.t. }((u, v) \in E) \wedge\left(\left|N^{-}(v)\right|=1\right) \wedge \\ & (v \text { belongs to a cluster } C \text { s.t. } w(u)+w(C) \leq M) \\ \delta_{G\left[V \backslash V_{0}\right]}+D \quad & \text { otherwise }\end{cases}
$$

Observe that the running time is $O\left(2^{\left|E\left(G\left[V \backslash V_{0}\right]\right)\right|}\right) \leq O\left(2^{2 \cdot\left|V \backslash V_{0}\right|}\right)=O\left(4^{\left|V \backslash V_{0}\right|}\right) \leq$ $O\left(4^{n}\right)$, where the first inequality is due to the fact that the outdegree of every vertex in $V \backslash V_{0}$ is at most two. 


\section{Chapter 6}

\section{Conclusion}

In this chapter, we summarize our main results and identify avenues for future work

\subsection{Summary of contributions}

In this dissertation, we studied the problems of disjoint clustering in combinatorial circuits for delay minimization $(\mathrm{CN})$. We obtained the computational complexities of several variants of $\mathrm{CN}$. We also showed that for some variants, our results imply hardness of approximation within a certain factor. Furthermore, we proposed some approximation and exact exponential algorithms and analyzed them. For some cases, we obtained an approximation factor of strictly less than two. Moreover, for some variants, our exact exponential algorithms beat brute force. Our inapproximability and approximability results for variants of $\mathrm{CN}$ that we have studied are tabulated in Table 6.1. In Figure 6.1, we present results for the original lattice of problems (cf. Figure 2.3. 
Table 6.1: Approximability and inapproximability results

\begin{tabular}{|l|c|c|c|}
\hline Problem & Approximability & Inapproximability & Running time \\
\hline $\mathrm{CN}\langle W, M, \Delta\rangle$ & $\left(M^{2}+M\right)$-approximable & $2-\varepsilon$ & $2^{O(\Delta \cdot M)} \cdot|V|^{O(1)}$ \\
\hline $\mathrm{CN}\langle W, M, 3\rangle$ & $\left(M^{2}+M\right)$-approximable & $2-\varepsilon$ & $2^{O(3 \cdot M)} \cdot|V|^{O(1)}$ \\
\hline $\mathrm{CN}\langle W, 2,3\rangle$ & 6 -approximable & $\frac{4}{3}-\varepsilon$ & $2^{6} \cdot|V|^{O(1)}$ \\
\hline $\mathrm{CN}\langle W, 2, \Delta\rangle$ & 6 -approximable & $\frac{4}{3}-\varepsilon$ & $2^{O(\Delta \cdot 2)} \cdot|V|^{O(1)}$ \\
\hline $\mathrm{CN}\langle N, M, \Delta\rangle$ & $\left(M^{2}+M\right)$-approximable & $\frac{3}{2}-\varepsilon$ & $2^{O(\Delta \cdot M)} \cdot|V|^{O(1)}$ \\
\hline $\mathrm{CN}\langle N, 2, \Delta\rangle$ & $\left(2+\frac{2}{l-1}\right)$-approximable & $\frac{3}{2}-\varepsilon$ & $O(\sqrt{n} \cdot m)$ \\
\hline $\mathrm{CN}\langle N, M, 3\rangle$ & $\left(M^{2}+M\right)$-approximable & $\frac{4}{3}-\varepsilon$ & $2^{O(3 \cdot M)} \cdot|V|^{O(1)}$ \\
\hline $\mathrm{CN}\langle N, 2,3\rangle$ & 2 -approximable & $\frac{4}{3}-\varepsilon$ & $O\left(T_{L P}(n, m)\right)$ \\
\hline
\end{tabular}




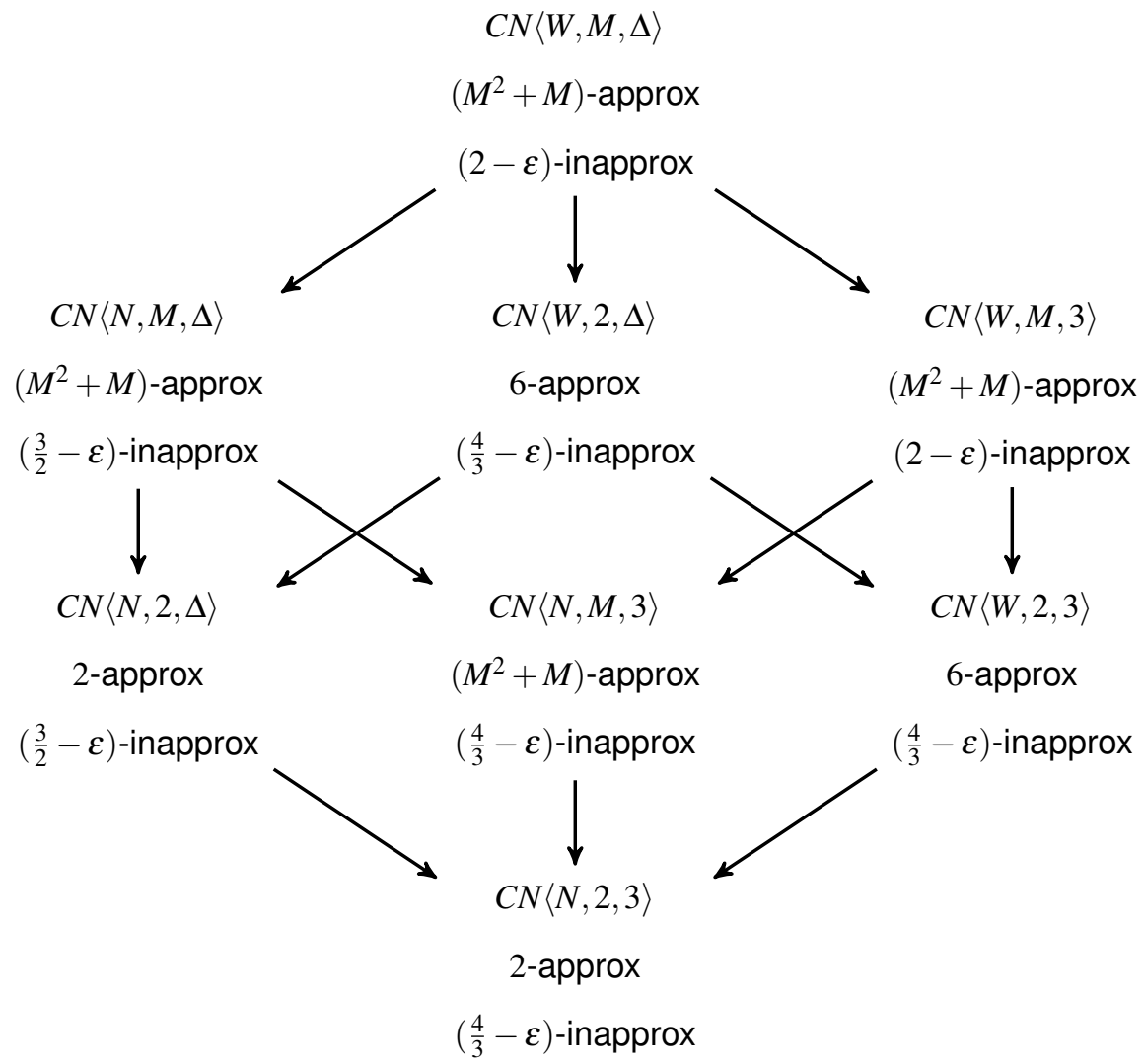

Figure 6.1: Results for some variants of the disjoint clustering problem that we investigated.

\subsection{Future work}

We are interested in further investigating the gap between the approximability and inapproximability of several variants of the problems that we have studied. To do so, we would like to prove inapproximability using different assumptions. In particular, we would work to obtain inapproximability results by assuming the truth of the UNIQUE GAMES CONJECTURE. In this effort, we hope to gain additional insights into the problems that we have studied. In turn, this may lead to some interesting algorithmic results. 


\subsubsection{Implementation and experimentation}

We have designed several algorithms for which we have also provided formal mathematical analyses to measure the quality of the solutions obtained, as well as to measure performance. We want to further this work by implementing our algorithms and obtaining experimental results. For instance, it would be interesting to see, after implementing our algorithms and carrying out several experiments on some benchmark circuits, how our experimental results compare to other algorithms and heuristics (e.g., the DJCL heuristic described in [Kag03]).

\subsubsection{Unique Games Conjecture}

Throughout our research, we based every inapproximability result on the common assumption that $\mathbf{P} \neq \mathbf{N P}$. However, there are other less traditional assumptions that we could have made. For example, we could have assumed the UNIQUE GAMES CONJECTURE to be true. In future work, we would like to prove inapproximability of several variants of some of the problems that we studied based on other assumptions. For that purpose, we begin this chapter by defining the 2-PROVER-1-Round (2P1R) GAME, a constraint satisfaction problem which motivated Khot's study of the UNIQUE GAMES (UG) Problem and lead to the Unique Games Conjecture (UGC) [Kho02]. The goal of the 2P1R GAME is to find an (approximately) optimal labeling to a given instance

$$
\mathscr{U}_{2 p 1 r}\left(G(V, W, E),[m],[n],\left\{\pi_{e} \mid e \in E\right\}\right),
$$

where $G(V, W, E)$ is a bipartite graph, each vertex $v \in V$ is assigned a label from the set $[m]$, each vertex $w \in W$ is a assigned a label from the set $[n]$, and a constraint associated with each edge $e=(v, w) \in E$, is represented by a surjective function $\pi_{e}:[m] \rightarrow[n]$ with $m \geq n[$ Kho10].

A 2P1R GAME can be thought of as a game between two provers, $P_{1}$ and $P_{2}$, and 
a probabilistic polynomial time verifier. The verifier randomly chooses an edge $e=$ $(v, w)$, from which vertices $v$ and $w$ are sent for labeling from the sets $[m]$ and $[n]$ (resp.) by provers $P_{1}$ and $P_{2}$, respectively. The verifier accepts if and only if the provers' answers satisfy $\pi_{e}(i)=j$, where $i$ and $j$ correspond to the labels given by $P_{1}$ and $P_{2}$, respectively. The probability of acceptance by the verifier is the same value as that of the provers' strategy [Kho10, KS13]. UG (as defined below) arises as a special case of 2P1R, when $m=n$, and the function $\pi_{e}:[m] \rightarrow[n]$ is bijective [Kho10].

Definition 6.2.1. A UNIQUE GAme $\mathscr{U}\left(G(V, E),[n],\left\{\pi_{e} \mid e \in E\right\}\right)$ is a constraint satisfaction problem defined as follows: $G(V, E)$ is a directed graph whose vertices represent variables and edges represent constraints. The goal is to assign to each vertex a label from the set $[n]$. The constraint on an edge $e=(v, w) \in E$ is described by a bijection $\pi_{e}:[n] \mapsto[n]$. A labeling $L: V \mapsto[n]$ satisfies the constraint on edge $e=(v, w)$ if and only if $\pi_{e}(L(v))=L(w)$. Let $O P T(\mathscr{U})$ denote the maximum fraction of constraints that can be satisfied by any labeling:

$$
O P T(\mathscr{U}):=\max _{L: V \mapsto[n]} \frac{1}{|E|} \cdot \mid\{e \in E \mid L \text { satisfies } e\} \mid
$$

For the purposes of illustration, consider the binary constraint satisfaction problem given by the maximum cut problem (MAX CUT). We recall MAX CUT as follows: Given an undirected graph $G=(V, E)$ and nonnegative weights $w_{i j} \geq 0$ for all edges $(i, j) \in E$, the goal is to find a partition of the vertices $S$ and $\bar{S}$ that maximizes the sum of the weights of the edges in the cut $(S, \bar{S})$. MAX CUT corresponds to the weighted constraint satisfaction problem in which $[n]=\{0,1\}$, there is a variable $x_{i}$ for each vertex $i \in V$, and there is a constraint $f\left(x_{i}, x_{j}\right)=x_{i} \oplus x_{j}$ for each edge $(i, j)$ with weight $w_{i j}$, where $\oplus$ is the exclusive-or function (which is 1 precisely when $x_{i} \neq x_{j}$ ). This problem happens to be a UG on a universe of two elements, because for any constraint on an edge $(i, j)$, when given the value of $x_{i} \in\{0,1\}$, there is only one value of $x_{j}$ for 
which the constraint is satisfied (likewise for a given value of $x_{j}$ ) [WS11].

The Unique Games Conjecture asserts that it is NP-hard to distinguish between instances of the unique games problem in which almost every constraint is satisfied and instances in which almost no constraint is satisfied. We now give a formal definition of the UnIQue GAMEs CONJECTURE [Kho02].

UniQUe Games CONJECTURE 1: For arbitrarily small constants $\zeta, \delta>0$, there exists a constant $k=k(\zeta, \delta)$ such that it is NP-hard to determine whether a unique 2-prover game with answers from a domain of size $k$ has value at least $1-\zeta$ or at most $\delta$.

Although still an open question, assuming that UGC is true was shown to imply optimal hardness results for many optimization problems [KKMO07, KR08, Rag08], and has motivated research leading to interesting algorithmic results [CMM06, Tre08, ABS15, Kol11]. Recent results are providing strong evidence towards correctness of $\mathrm{UGC}\left[\mathrm{DKK}^{+} 18 \mathrm{~b}, \mathrm{DKK}^{+} 18 \mathrm{a}, \mathrm{KMS}^{\mathrm{N}}\right]$ ]. 


\section{Bibliography}

[AB10] Mikhail J. Atallah and Marina Blanton. Algorithms and theory of computation handbook. CRC Press, Boca Raton, 2nd ed. / edition, 2010.

[ABS15] Sanjeev Arora, Boaz Barak, and David Steurer. Subexponential algorithms for unique games and related problems. J. ACM, 62(5):42:1-42:25, 2015.

[AFIM06] Yuichi Asahiro, Tetsuya Furukawa, Keiichi Ikegami, and Eiji Miyano. How to pack directed acyclic graphs into small blocks. In Tiziana Calamoneri, Irene Finocchi, and Giuseppe F. Italiano, editors, Algorithms and Complexity, pages 272-283, Berlin, Heidelberg, 2006. Springer Berlin Heidelberg.

[AMY18] Yuichi Asahiro, Eiji Miyano, and Tsuyoshi Yagita. Approximation algorithms for packing directed acyclic graphs into two-size blocks. In Osvaldo Gervasi, Beniamino Murgante, Sanjay Misra, Elena Stankova, Carmelo M. Torre, Ana Maria A.C. Rocha, David Taniar, Bernady O. Apduhan, Eufemia Tarantino, and Yeonseung Ryu, editors, Computational Science and Its Applications - ICCSA 2018, pages 607-623, Cham, 2018. Springer International Publishing.

[Arg01] Lars Arge. External memory data structures. In Algorithms - ESA 2001, 9th Annual European Symposium, Aarhus, Denmark, August 28-31, 2001, Proceedings, pages 1-29, 2001.

[AV88] Alok Aggarwal and Jeffrey Scott Vitter. The input/output complexity of sorting and related problems. Commun. ACM, 31(9):1116-1127, 1988.

[BCLS87] T. N. Bui, S. Chaudhuri, F. T. Leighton, and M. Sipser. Graph bisection algorithms with good average case behavior. Combinatorica, 7(2):171191, Jun 1987.

[BJ89] T. N. Bui and C. Jones. Sequential and parallel algorithms for partitioning simple classes of graphs. Technical report, Department of Computer Science, The Pennsylvania State University, University Park, Pennsylvania, 1989. 
[BJG10] Jørgen Bang-Jensen and Gregory Gutin. Digraphs : Theory, Algorithms and Applications. Springer, London, 2010.

[CD94] J. Cong and Yuzheng Ding. FlowMap: an optimal technology mapping algorithm for delay optimization in lookup-table based FPGA designs. IEEE Transactions on Computer-Aided Design of Integrated Circuits and Systems, 13(1), 1994.

$\left[\mathrm{CFK}^{+} 15\right]$ Marek Cygan, Fedor V. Fomin, Łukasz Kowalik, Daniel Lokshtanov, Daniel Marx, Marcin Pilipczuk, Michał Pilipczuk, and Saket Saurabh. Parameterized algorithms. Springer, Cham, 2015.

[CLRS09] Thomas H. Cormen, Charles E. Leiserson, Ronald L. Rivest, and Clifford Stein. Introduction to Algorithms, Third Edition. The MIT Press, 3rd edition, 2009.

[CMM06] Moses Charikar, Konstantin Makarychev, and Yury Makarychev. Nearoptimal algorithms for unique games. In Proceedings of the 38th Annual ACM Symposium on Theory of Computing, Seattle, WA, USA, May 21-23, 2006, pages 205-214, 2006.

[CR01] J. Cong and M. Romesis. Performance-driven multi-level clustering with application to hierarchical fpga mapping. In Proceedings of the 38th Design Automation Conference (IEEE Cat. No.01CH37232), pages 389394, June 2001.

[DF09] Richard Denman and Stephen Foster. Using clausal graphs to determine the computational complexity of k-bounded positive one-in-three "SAT". Discrete Applied Mathematics, 157(7):1655-1659, 2009.

[DGMS18] Zola Donovan, Gregory Z. Gutin, Vahan Mkrtchyan, and K. Subramani. Clustering without replication in combinatorial circuits. Accepted pending revision, 2018.

$\left[\mathrm{DKK}^{+} 18 \mathrm{a}\right]$ Irit Dinur, Subhash Khot, Guy Kindler, Dor Minzer, and Muli Safra. On non-optimally expanding sets in grassmann graphs. In Proceedings of the 50th Annual ACM SIGACT Symposium on Theory of Computing, STOC 2018, Los Angeles, CA, USA, June 25-29, 2018, pages 940-951, 2018.

$\left[\mathrm{DKK}^{+} 18 \mathrm{~b}\right]$ Irit Dinur, Subhash Khot, Guy Kindler, Dor Minzer, and Muli Safra. Towards a proof of the 2-to-1 games conjecture? In Proceedings of the 50th Annual ACM SIGACT Symposium on Theory of Computing, STOC 2018, Los Angeles, CA, USA, June 25-29, 2018, pages 376-389, 2018.

[DMS15] Zola Donovan, Vahan Mkrtchyan, and K. Subramani. On clustering without replication in combinatorial circuits. In Combinatorial Optimization and Applications - 9th International Conference, COCOA 2015, Houston, TX, USA, December 18-20, 2015, Proceedings, pages 334-347, 2015. 
[DRSS96] Ajit A. Diwan, Sanjeeva Rane, S. Seshadri, and S. Sudarshan. Clustering techniques for minimizing external path length. In VLDB'96, Proceedings of 22th International Conference on Very Large Data Bases, September 3-6, 1996, Mumbai (Bombay), India, pages 342-353, 1996.

[FK10] Fedor V. Fomin and Dieter Kratsch. Exact Exponential Algorithms. Texts in Theoretical Computer Science. An EATCS Series. Springer, 2010.

[GH88] O. Goldschmidt and D. S. Hochbaum. Polynomial algorithm for the k-cut problem. In [Proceedings 1988] 29th Annual Symposium on Foundations of Computer Science, pages 444-451, Oct 1988.

[GM88] M. Goldberg and Z. Miller. A parallel algorithm for bisection width in trees. Computers and Mathematics with Applications, 15(4):259-266, 1988.

[HG95] L. J. Hwang and A. El Gamal. Min-cut replication in partitioned networks. IEEE Transactions on Computer-Aided Design of Integrated Circuits and Systems, 14(1), 1995.

[Kag03] Dimitri Kagaris. On minimum delay clustering without replication. Integration, the VLSI Journal, 36(1):27-39, 2003.

[Kao15] Chi-Chou Kao. Clock skew minimization in multiple dynamic supply voltage with adjustable delay buffers restriction. Journal of Signal Processing Systems : for Signal, Image, and Video Technology (formerly the Journal of VLSI Signal Processing Systems for Signal, Image, and Video Technology), 79(1):99-104, 2015.

[Kho02] Subhash Khot. On the power of unique 2-prover 1-round games. In Proceedings on 34th Annual ACM Symposium on Theory of Computing, May 19-21, 2002, Montréal, Québec, Canada, pages 767-775, 2002.

[Kho10] S. Khot. On the unique games conjecture (invited survey). In 2010 IEEE 25th Annual Conference on Computational Complexity, pages 99-121, June 2010.

[KKMO07] Subhash Khot, Guy Kindler, Elchanan Mossel, and Ryan O’Donnell. Optimal inapproximability results for MAX-CUT and other 2-variable csps? SIAM J. Comput., 37(1):319-357, 2007.

[KMS18] Subhash Khot, Dor Minzer, and Muli Safra. Pseudorandom sets in grassmann graph have near-perfect expansion. Electronic Colloquium on Computational Complexity (ECCC), 25:6, 2018.

[Kol11] Alexandra Kolla. Spectral algorithms for unique games. Computational Complexity, 20(2):177-206, 2011. 
[Kos04] Thomas Koshy. Discrete Mathematics with Applications. Elsevier Science \& Technology, San Diego, 2004.

[KR08] Subhash Khot and Oded Regev. Vertex cover might be hard to approximate to within 2-epsilon. J. Comput. Syst. Sci., 74(3):335-349, 2008.

[KS13] Subhash Khot and Muli Safra. A two-prover one-round game with strong soundness. Theory of Computing, 9:863-887, 2013.

[LLT69] E. L. Lawler, K. N. Levitt, and J. Turner. Module clustering to minimize delay in digital networks. IEEE Transactions on Computers, 18(1), 1969.

[Mac88] R. M. MacGregor. On partitioning a graph: A theoretical and empirical study. PhD thesis, University of California, Berkeley, 1988.

[MBSV91] R. Murgai, R. K. Brayton, and A. Sangiovanni-Vincentelli. On clustering for minimum delay/area. In 1991 IEEE International Conference on Computer-Aided Design Digest of Technical Papers, pages 6-9, 1991.

[ML14] R. Manikandan and V. Leela. Effective clustering algorithms for VLSI circuit partitioning problems. Contemporary Engineering Sciences, 7(1720):923-929, 2014.

[MR01] C. Moore and J.M. Robson. Hard tiling problems with simple tiles. Discrete \& Computational Geometry, 26(4):573-590, Jan 2001.

[MW96] Wai-Kei Mak and D. F. Wong. Minimum replication min-cut partitioning. In Proceedings of International Conference on Computer Aided Design, pages 205-210, 1996.

[MWK15] Konstantin Moiseev, Shmuel Wimer, and Avinoam Kolodny. Timingconstrained power minimization in VLSI circuits by simultaneous multilayer wire spacing. Integration, the VLSI Journal, 48(4):116-128, 2015.

[MZ02] Anil Maheshwari and Norbert Zeh. A survey of techniques for designing i/o-efficient algorithms. In Algorithms for Memory Hierarchies, Advanced Lectures [Dagstuhl Research Seminar, March 10-14, 2002], pages 36-61, 2002.

[Pap94] Christos H. Papadimitriou. Computational complexity. Addison-Wesley, Reading, Massachusetts, 1994.

[Rag08] Prasad Raghavendra. Optimal algorithms and inapproximability results for every csp? In Proceedings of the 40th Annual ACM Symposium on Theory of Computing, Victoria, British Columbia, Canada, May 17-20, 2008, pages 245-254, 2008.

[RW93] R. Rajaraman and D. F. Wong. Optimal clustering for delay minimization. In 30th ACM/IEEE Design Automation Conference, pages 309-314, 1993. 
[SG11] I. Hameem Shanavas and Ramaswamy Kannan Gnanamurthy. Wirelength minimization in partitioning and floorplanning using evolutionary algorithms. VLSI Design, 2011(4):1-9, 2011.

[SG14] I. Hameem Shanavas and R. K. Gnanamurthy. Optimal solution for VLSI physical design automation using hybrid genetic algorithm. Mathematical Problems in Engineering, 2014(4):1-15, 2014.

[SHY $\left.{ }^{+} 10\right] \quad Y . S$ Su, W. K Hon, C. C Yang, S. C Chang, and Y. J Chang. Clock skew minimization in multi-voltage mode designs using adjustable delay buffers. IEEE Transactions on Computer-Aided Design of Integrated Circuits and Systems, 29(12):1921-1930, 2010.

[SPMS02] Amit Singh, Ganapathy Parthasarathy, and Malgorzata Marek-Sadowska. Efficient circuit clustering for area and power reduction in FPGAs. ACM Transactions on Design Automation of Electronic Systems, 7(4):643-663, 2002.

[SSA10] P. Subbaraj, S. Saravanasankar, and S. Anand. Combinatorial optimization in VLSI hypergraph partitioning using taguchi methods. International Journal of Mathematical Combinatorics, 3:69-84, 2010.

[Tre08] Luca Trevisan. Approximation algorithms for unique games. Theory of Computing, 4(1):111-128, 2008.

[Vit98] Jeffrey Scott Vitter. External memory algorithms. In Gianfranco Bilardi, Giuseppe F. Italiano, Andrea Pietracaprina, and Geppino Pucci, editors, Algorithms - ESA' 98, pages 1-25, Berlin, Heidelberg, 1998. Springer Berlin Heidelberg.

[Vit01] Jeffrey Scott Vitter. External memory algorithms and data structures. ACM Comput. Surv., 33(2):209-271, 2001.

[Vit06] Jeffrey Scott Vitter. Algorithms and data structures for external memory. Foundations and Trends in Theoretical Computer Science, 2(4):305-474, 2006.

[VV96] Darren Erik Vengroff and Jeffrey Scott Vitter. I/o-efficient algorithms and environments. ACM Comput. Surv., 28(4es):212, 1996.

[Wes01] Douglas Brent West. Introduction to graph theory. Prentice Hall, Upper Saddle River, N.J., 2nd ed. edition, 2001.

[WS11] David P. Williamson and David B. Shmoys. The Design of Approximation Algorithms. Cambridge University Press, 2011.

[YW97] H. H. Yang and D. F. Wong. Circuit clustering for delay minimization under area and pin constraints. IEEE Transactions on Computer-Aided Design of Integrated Circuits and Systems, 16(9):976-986, Sep 1997. 